\title{
Repetitive Operation of the University of Saskatchewan Compact Torus INJECTOR
}

\author{
A Thesis Submitted to the \\ College of Graduate Studies and Research \\ in Partial Fulfillment of the Requirements \\ for the degree of Master of Science \\ in the Department of Physics and Engineering Physics \\ University of Saskatchewan \\ Saskatoon \\ By \\ Arun Andre Pant \\ (C)Arun Andre Pant, July 2009. All rights reserved.
}




\section{Permission TO Use}

In presenting this thesis in partial fulfilment of the requirements for a Postgraduate degree from the University of Saskatchewan, I agree that the Libraries of this University may make it freely available for inspection. I further agree that permission for copying of this thesis in any manner, in whole or in part, for scholarly purposes may be granted by the professor or professors who supervised my thesis work or, in their absence, by the Head of the Department or the Dean of the College in which my thesis work was done. It is understood that any copying or publication or use of this thesis or parts thereof for financial gain shall not be allowed without my written permission. It is also understood that due recognition shall be given to me and to the University of Saskatchewan in any scholarly use which may be made of any material in my thesis.

Requests for permission to copy or to make other use of material in this thesis in whole or part should be addressed to:

Head of the Department of Physics and Engineering Physics

116 Science Place

University of Saskatchewan

Saskatoon, Saskatchewan

Canada

S7N 5E2 


\section{Abstract}

Development of fueling technologies for modern and future tokamak reactors is essential for their implementation in a commercial energy production setting. Compared to the presently available fueling technologies, gas or cryogenic pellet injection, compact torus injection presents an effective and efficient method for directly fueling the central core of tokamak plasmas. Fueling of the central core of a tokamak plasma is pivotal for providing efficient energy production. The central core plasma of a reactor contains the greatest density of fusion processes. For consistent and continuous fueling of tokamak fusion reactors, compact torus injectors must be operated in a repetitive mode.

The goal of this thesis was to study the feasibility of firing the University of Saskatchewan Compact Torus Injector (USCTI) in a repetitive mode. In order to enable USCTI to fire repetitively, modifications were made to its electrical system, control system and data acquisition system. These consisted primarily of the addition of new power supplies, to enable fast charging of the many capacitor banks used to form and accelerate the plasma. The maximum firing rate achieved on USCTI was $0.33 \mathrm{~Hz}$, an increase from the previous maximum firing rate of $0.2 \mathrm{~Hz}$ achieved at UC Davis.

Firing USCTI in repetitive modes has been successful. It has been shown that the CTs produced in any given repetitive series are properly formed and repeatable. This is made evident through analysis of data collected from the CTs' magnetic fields and densities as they traveled along the injector barrel. The shots from each experiment were compared to the series' mean data and were shown to be consistent over time. Calculations of their correlations show that there are only minimal deviations from shot to shot in any given series. 


\section{ACKnowledgements}

Foremost, I would like to thank my supervisor, Professor Chijin Xiao. His guidance, dedication and expertise, not only as a supervisor, but as a teacher, through all stages of this project proved to be invaluable. I will take many lessons I have learned from him, with me throughout my career.

I am also grateful to David McColl. I have greatly benefited from Dave's experience in the laboratory and on the workbench and from his kind words. He has spent countless hours assisting me on finding creative solutions to various problems encountered on the USCTI system.

Dr. Dazhi Liu's familiarity with USCTI was also an invaluable resource during the final stages of the project.

I would like to thank my colleagues, Dr. Nathan Joiner, Damian Rohraff, Dallas Trembach, Sayf Elgriw and Koloman Varady for providing a friendly atmosphere, conducive to research, and for acting as a sounding board in the development of the ideas for my project.

This work was supported through grants from the Natural Sciences and Engineering Research Council of Canada and from the University of Saskatchewan Graduate Teaching Fellowship.

I cannot thank my parents enough. Their support, encouragement and love has helped me beyond measure.

Finally, I would like to thank Françoise. Were it not for her care, consideration, patience and emotional support, this project would not have been possible. 
...to nonna, akka, nonno and apakaka. 


\section{Contents}

Permission to Use $\quad$ i

Abstract $\quad$ ii

Acknowledgements $\quad$ iii

Contents $\quad$ v

List of Tables vii

List of Figures viii

List of Abbreviations $\quad$ xi

List of Symbols $\quad$ xii

1 Introduction 1

1.1 Energy Crisis . . . . . . . . . . . . . . . . . . . 1

1.2 Nuclear Fusion and the Tokamak Reactor . . . . . . . . . . . . . . . . 1

1.3 Tokamak Fueling and the Compact Toroid Injector . . . . . . . . . . 8

1.4 Motivation for Repetitive Compact Torus Operation . . . . . . . . . . 12

1.5 Thesis Outline . . . . . . . . . . . . . . . . . 13

2 Compact Torus Physics 15

2.1 Basic Concept . . . . . . . . . . . . . . . . . . . . 15

2.2 Magnetic Structure of the Compact Torus . . . . . . . . . . . . . . . 16

2.3 Formation and Acceleration . . . . . . . . . . . . . . . . 19

2.3.1 Formation ................... 19

2.3.2 Acceleration ..................... 19

2.4 Compact Torus Injection . . . . . . . . . . . . . . . . . . . . . . . . . . . . . . . . 21

2.4.1 Compact Torus Lifetime . . . . . . . . . . . . . . . 22

2.4.2 Tokamak Penetration . . . . . . . . . . . . . . . 23

2.4.3 Magnetic Reconnection and Fuel Deposition . . . . . . . . . . 24

2.5 Summary . . . . . . . . . . . . . . . . . . 25

3 Repetitive Operation of the USCTI Injector 26

3.1 USCTI . . . . . . . . . . . . . . . . . . . . . 26

3.1 .1 Basic Injector Design . . . . . . . . . . . . . . . . . 26

3.1 .2 Vacuum Systems and Gas Injection . . . . . . . . . . . . . 28

3.1.3 Electrical System . . . . . . . . . . . . . . . . . . 30

3.2 Modifications to USCTI . . . . . . . . . . . . . . . . . . . . . . . . . . . . . . . . 31

3.2.1 Main Power Supplies . . . . . . . . . . . . . . . . . . . 32 
3.2 .2 Grounding and Relays . . . . . . . . . . . . . . . . 33

3.2 .3 Krytrons . . . . . . . . . . . . . . . . . . . 38

3.2 .4 Gas Valves . . . . . . . . . . . . . . . . . . . . . . . . . . 40

3.3 Summary . . . . . . . . . . . . . . . . . . . . . 41

4 Diagnostics and Data Analysis 42

4.1 Diagnostic Theory . . . . . . . . . . . . . . . . . . 43

4.1.1 Magnetic and Current Probes . . . . . . . . . . . . . 45

4.1.2 Density Measurements . . . . . . . . . . . . . . . 48

4.1.3 Assumptions to Langmuir Probe Theory . . . . . . . . . . . . 51

4.1.4 Triple Langmuir Probe . . . . . . . . . . . . . . . . . . 52

4.1.5 Interferometry . . . . . . . . . . . . . . . . 55

4.2 Data Acquisition and Analysis . . . . . . . . . . . . . 56

4.3 Summary . . . . . . . . . . . . . . . . . . . . . 59

5 Experimental Results $\quad 60$

5.1 Preliminary Results . . . . . . . . . . . . . . . . . . . . . 61

5.2 Long Term Repetition . . . . . . . . . . . . . . . . . . . . . . . 63

5.3 Density Measurements . . . . . . . . . . . . . . . . . . . 67

5.4 Higher Repetition Rate . . . . . . . . . . . . . . . . . . . 71

5.5 Time-of-Flight Analysis . . . . . . . . . . . . . . . . . . . . 75

5.6 Summary . . . . . . . . . . . . . . . . . . 76

6 Conclusions and Future Work $\quad 77$

6.1 Summary and Conclusions . . . . . . . . . . . . . . 77

6.2 Future Work . . . . . . . . . . . . . . . . . . . . . 79

$\begin{array}{ll}\text { References } & 85\end{array}$

$\begin{array}{ll}\text { A Data Acquisition and Analysis Code } & 86\end{array}$ 


\section{LIST OF TABLES}

1.1 Parameters of the STOR-M tokamak. . . . . . . . . . . . 8

1.2 Parameters of $\mathrm{CT}$ at exit of accelerator. . . . . . . . . . . . . 11

3.1 Typical capacitor bank settings and charging times before and after modifications. . . . . . . . . . . . . . . . . 31 


\section{List OF Figures}

1.1 A diagram of the process of an inertial confinement fusion device. a) lasers bombard the target fuel pellet b) the outer layer of fuel breaks down into a plasma, detonating outward and compressing inward c) the inner gas also forms a plasma, following the detonation shockwave d) once sufficient temperature and density are achieved, fusion reactions are achieved. . . . . . . . . . . . . . 2

1.2 An artist's representation of the future Wendelstein VII stellarator. . 5

1.3 A general diagram of a tokamak showing the poloidal and toroidal magnetic coils and the resultant magnetic fields as well as the iron core transformer. Plasma current, $I_{p}$ flows in the counter-clockwise direction (from a top view). . . . . . . . . . . . . . 6

1.4 Schematic of the STOR-M tokamak, showing Ohmic coils OH, vertical equilibrium coils VE, toroidal coils and feedback coils FB. . . . . . . 7

1.5 General schematic of a CT injector, showing formation, compression and acceleration regions. . . . . . . . . . . . . . .

2.1 Diagram of CT magnetic fields. In the case of USCTI, the inner acceleration electrode is located along the $\hat{z}$ axis. . . . . . . . . . .

2.2 A typical formation section for a CT accelerator, a) the solenoid is energized, b) gas is injected in the formation section, c) the formation bank is discharged, breaking down the gas, d) after the CT is pushed through the solenoid field, a poloidal field remains in the CT. . . . . .

2.3 Motion of a CT along an accelerator. $\vec{B}$ is azimuthal, $\vec{J}$ is radial and $\vec{J} \times \vec{B}$ is to the right. . . . . . . . . . . . . . .

2.4 The CT as it a) is accelerated through the injector b) tilts in the tokamak magnetic field and c) reconnects at the fuel deposition point

3.1 USCTI cross section, depicting locations of the gas valves, diagnostic ports as well as formation and acceleration regions. . . . . . . . . .

3.2 Diagrams of the gas valves showing a) the general valve schematic and b) an expanded schematic of the spring/piston system. . . . . . . . .

3.3 Schematic of the charging and discharging system for the formation and acceleration capacitor banks. . . . . . . . . . . . . . .

3.4 Diagram of the current waveforms for the formation and acceleration discharges. . . . . . . . . . . . . . . . .

3.5 Schematic of the grounding relays on the formation and acceleration capacitor banks (both banks are similar). The capacitors which enable prolongation of the power supply inhibit signal as well as open ground are shown. The figure in the upper left corner shows the scheme for the Ross relay and inhibit signal overlapping. . . . . . . . . . . . . 
3.6 The krytron charging and discharging system. The charging resistor, which was changed to allow faster charging, is shown surrounded by

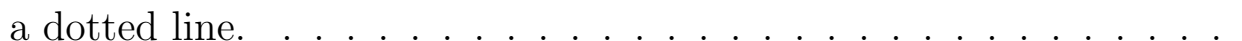

4.1 A schematic of the USCTI acceleration region, showing the locations of the probes used. . . . . . . . . . . . . . . . . . . . 44

4.2 Schematic of the integrators used on USCTI. . . . . . . . . . . . . . 45

4.3 Diagram of a rogowski coil showing the current $I$, going into the page and causing the magnetic field $\mathbf{B}$ at the Rogowski coil of radius $R$ and cross sectional area, A. . . . . . . . . . . . . . . . . . 47

4.4 Schematic of a self-integrator used on the Rogowski coils. The resistor $r$ is the internal resistance of the probe. . . . . . . . . . . . . . . . . 48

4.5 Typical I-V curve showing a) ion saturation current region, b) floating potential $V_{f}$ and transition region and c) electron saturation current region. . . . . . . . . . . . . . . . . . 49

4.6 A basic triple probe circuit. . . . . . . . . . . . . . . . . . 53

4.7 Diagram showing the arrangement of the triple probe used on USCTI. 54

4.8 Basic diagram of interferometer used on USCTI. . . . . . . . . . . . . 56

4.9 An example of a mean shot from poloidal magnetic field, $B_{z}$, measurements as well as a well correlated sample shot, 994 (with a correlation coefficient of $\sim 0.98$ ) and a poorly correlated shot, 1008 (with a correlation coefficient of $\sim 0.58$ ) which have been excluded in calculations of the best case mean.

$5.1 B_{z}$ probe data from shot numbers a) 576 and b) 581, in a 20 second interval firing. The time-of-flight of the $\mathrm{CT}$ in shot number 576 as it passes the $\mathrm{z}=43 \mathrm{~cm}$ and $\mathrm{z}=65 \mathrm{~cm}$ probes is shown. . . . . . . . .

5.2 a) mean $B_{z}$ probe data from shot numbers 574 - 584 as well as b) correlation coefficients of each shot with respect to their mean. . . . .

5.3 Shot numbers 992 - 1091, a) shot 1021 and B) mean $B_{z}$ probe data as well as c) correlation coefficients of each shot with respect to their mean.

5.4 Graphs of the a) mean and b) correlation coefficients of the formation and acceleration current waveforms for the 992-1091 shot series. . . .

5.5 Triple Langmuir probe data from preliminary density measurements. $T_{e}, I_{\text {sat }}$ and $n_{e}$ are presented from a) shot 1253 and b) the mean from shots 1237 to $1255 . \ldots$. . . . . . . . . . . . . .

5.6 a) shot 1585 and b) mean $B_{z}$ and $n_{e}$ probe data from the $1582-1591$

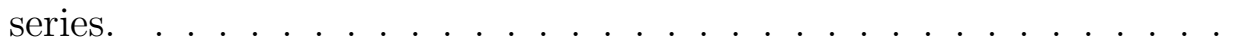

5.74 second interval data from shot 1615 to 1621 . a) a sample shot, 1616 and b) mean $B_{z}$ and $n_{e}$ probe data are presented. . . . . . . . . . . . 72

5.83 second interval data from shot 1640 to 1645 . A) a sample shot, 1641 and b) mean $B_{z}$ and $n_{e}$ probe data are presented. . . . . . . . . . . 73

5.9 Correlation of high repetition measurements, a) 4 second and b) 3 second interval firings. . . . . . . . . . . . . . . . . 
5.10 Velocities of the CTs from 3 second interval repetition experiments. . $\quad 75$

A.1 Labview acquisition software. . . . . . . . . . . . . . . . 94 


\section{List of ABBREviAtions}

$\begin{array}{ll}\text { CT } & \text { Compact Torus } \\ \text { CTIX } & \text { Compact Torus Injection Experiment } \\ \text { FWHM } & \text { Full Width at Half Maximum } \\ \text { ITER } & \text { International Thermonuclear Experimental Reactor } \\ \text { JET } & \text { Joint European Torus } \\ \text { LLNL } & \text { Lawrence Livermore National Laboratory } \\ \text { RACE } & \text { Ring Accelerator Experiment } \\ \text { RF } & \text { Radio Frequency } \\ \text { SCR } & \text { Silicon Controlled Rectifier } \\ \text { STOR-M } & \text { Saskatchewan Torus Modified } \\ \text { TdeV } & \text { Tokamak de Varrennes } \\ \text { TMP } & \text { Turbo Molecular Pump } \\ \text { UC } & \text { University of California } \\ \text { USCTI } & \text { University of Saskatchewan Compact Torus Injector } \\ \text { OFHC } & \text { Oxygen Free High Conducting }\end{array}$




\section{List of Symbols}

\begin{tabular}{|c|c|}
\hline$\Gamma$ & Secondary Emission Rate \\
\hline$\eta$ & Spitzer Resistivity \\
\hline$\lambda_{D}$ & Debye Length \\
\hline$\mu_{0}$ & Permeability of Free Space \\
\hline$\nu_{e i}$ & Electron-Ion Collision Frequency \\
\hline$\rho$ & Mass Density \\
\hline$\tau_{E}$ & Confinement Time \\
\hline$\tau_{\text {life }}$ & Compact Torus Lifetime \\
\hline$\tau_{p}$ & Compact Torus Penetration Time \\
\hline$\Phi$ & Magnetic Flux \\
\hline$\phi_{s}$ & Plasma Sheath Potential \\
\hline$\omega$ & Electromagnetic Wave Frequency \\
\hline$\omega_{p}$ & Plasma Frequency \\
\hline$B_{r}, B_{\theta}, B_{z}$ & Compact Torus Radial, Toroidal and Poloidal Magnetic Field \\
\hline & Cut-Off Frequency \\
\hline$I_{p}$ & Plasma Current \\
\hline$I_{s}$ at & Ion Saturation Current \\
\hline$J_{e 0}, J_{i 0}$ & Electron and Ion Saturation Current Density \\
\hline$L_{p}$ & Compact Torus Stopping Point \\
\hline$m_{e}, m_{i}$ & Electron and Ion Mass \\
\hline$n$ & Particle Density \\
\hline$n_{0}$ & Plasma Density \\
\hline$n_{e}$ & Electron Density \\
\hline$P$ & Thermal Pressure \\
\hline$P_{\text {peak }}$ & Peak Power of Power Supply \\
\hline$R, a$ & Major and Minor Radii \\
\hline$r_{C T}$ & Compact Torus Radius \\
\hline$r_{e}, r_{i}$ & Electron and Ion Larmor Radii \\
\hline$r_{l}$ & Larmor Radius \\
\hline$T_{\text {charge }}$ & Charging Time \\
\hline$T_{e}$ & Electron Temperature \\
\hline$V_{\text {accel }}$ & Acceleration Bank Voltage \\
\hline$V_{d 2}$ & Langmuir Triple Probe Temperature Output Signal \\
\hline$V_{f}$ & Probe Floating Potential \\
\hline$V_{\text {form }}$ & Formation Bank Voltage \\
\hline$V_{\max }$ & Maximum Voltage of Power Supply \\
\hline$V_{s}$ & Plasma Space Potential \\
\hline$v_{A}$ & Alfvén Velocity \\
\hline$v_{C T}$ & Compact Torus Velocity \\
\hline$v_{T e}$ & Electron Thermal Velocity \\
\hline$Z_{\text {eff }}$ & Effective Charge \\
\hline
\end{tabular}




\section{Chapter 1}

\section{INTRODUCTION}

\section{$1.1 \quad$ Energy Crisis}

Increasing attention towards environmental sustainability, rising global demands for energy, and diminishing fossil fuel reserves have brought about the desire for better energy sources. Fusion power presents the greatest source for massive amounts of power with a limited ecological footprint $[1,2]$. Commercial fusion power would be of benefit to humanity for several reasons. It would be environmentally superior to fossil fuels; unlike renewable sources, such as wind and solar power, it would be able to provide base load energy for large populated areas and lastly, it would have lower amounts of radioactive by-products than conventional nuclear fission reactors.

World wide research to develop a viable fusion energy source has been ongoing since the early 1950s. Much of the earlier work was an offshoot of weapons programs and was classified. By the late 1950s fusion research was declassified and subsequently multinational collaborative programs began to take shape in response to the world's energy needs.

\subsection{Nuclear Fusion and the Tokamak Reactor}

Fusion power, in itself, is not a novel concept; the largest known source of energy, the Sun, is a nuclear fusion reactor. There are two classes of fusion reactors being explored on Earth, inertial and magnetic. Inertial confinement fusion reactors im-

plement lasers to simultaneously heat, breakdown and confine fusion fuel. Lasers are used to heat and breakdown the outer layer of a small fuel capsule or pellet. The 
expanding outer layer of plasma creates an inward pressure and transfer of energy. This serves to compress and heat the inner part of the fuel. The exploding outer layer of plasma also creates shock waves which travel at very high velocity to the central region of the fuel pellet, raising the temperature and density even further. The subsequent high density in the innermost part of the fuel pellet leads to an initiation of fusion reactions. Problems related to inertial confinement include target positioning, fueling of the target, repetitive laser pulse firing as well as controlling the symmetry of the imploding shock wave. Figure 1.1 [3], shows a simplistic example of the inertial confinement reaction.

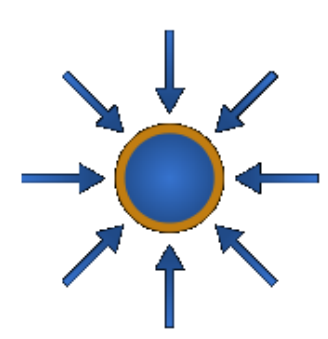

a)

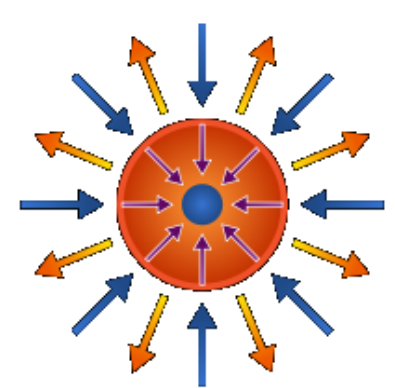

b)

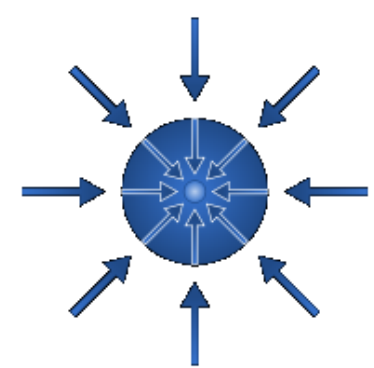

c)

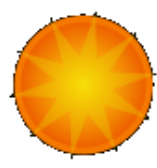

d)

Figure 1.1: A diagram of the process of an inertial confinement fusion device. a) lasers bombard the target fuel pellet b) the outer layer of fuel breaks down into a plasma, detonating outward and compressing inward c) the inner gas also forms a plasma, following the detonation shockwave d) once sufficient temperature and density are achieved, fusion reactions are achieved.

Although progress is being made in inertial confinement fusion, it is magnetic confinement that is showing the greatest promise. Of the many devices, it is the tokamak reactor which is showing the greatest promise.

The most heavily explored and promising energy producing fusion reaction, first tested at the Joint European Torus (JET) [4], is the Deuterium-Tritium reaction, as it has the largest fusion cross section. When Deuterium and Tritium are combined together, the resulting products are an alpha particle, a neutron and 17.6 MeV of 
energy.

$$
\mathrm{D}^{2}+\mathrm{T}^{3} \rightarrow \mathrm{He}^{4}+\mathrm{n}^{1}+17.6 \mathrm{MeV}
$$

In a tokamak fusion reactor, at densities $n \gtrsim 10^{20} \mathrm{~m}^{-3}$ and temperatures $T \gtrsim$ $10 \mathrm{keV}$, this reaction occurs frequently enough to produce significant amounts of fusion energy.

A commonly referred to expression of the requirements for fusion reactions to take place is the triple product of density, $n$, temperature, $T$, and confinement time, $\tau_{E}, n \cdot T \cdot \tau_{E}$. The confinement time is defined as

$$
\tau_{E}=\frac{W}{P_{\text {loss }}},
$$

the ratio of $W$, the energy content of the system, and $P_{l o s s}$, the rate of power loss for the system. In a $\mathrm{D}$ - $\mathrm{T}$ reaction, the value for the triple product required for a fusion reactor is $n \cdot T \cdot \tau_{E} \geq 10^{21} \mathrm{keV} \mathrm{s} / \mathrm{m}^{3}$.

Another useful measure of fusion reactors is what is commonly referred to as the $Q$ value, or fusion energy gain factor. This is essentially a ratio between the amount of power produced in a fusion reactor versus the amount of power required to sustain the reaction itself. The International Thermonuclear Experimental Reactor (ITER), currently being constructed in France, is planned to be the world's first break even reactor. Break even corresponds to $Q=1$.

Deuterium can be harvested from water as $\mathrm{D}_{2} \mathrm{O}$, with an abundance of $100 \mathrm{ppm}$, however tritium is an unstable element and does not exist abundantly in nature, with abundances of less than 100 parts per $10^{18}$. Tritium is therefore produced in a process called breeding. A neutron is absorbed into the lithium isotope $\mathrm{Li}^{6}$, with abundances of $75000 \mathrm{ppm}$ in natural lithium, or $\mathrm{Li}^{7}$. The $\mathrm{Li}^{7}$ reaction, however, consumes energy as opposed to producing it, [5].

$$
\begin{aligned}
& \mathrm{Li}^{6}+\mathrm{n}^{1} \rightarrow \mathrm{He}^{4}+\mathrm{T}^{3}+4.8 \mathrm{MeV} \\
& \mathrm{Li}^{7}+\mathrm{n}^{1} \rightarrow \mathrm{He}^{4}+\mathrm{T}^{3}+\mathrm{n}^{1}-2.5 \mathrm{MeV}
\end{aligned}
$$

The charged He particle, from Equation 1.3, will also heat the fuel in the reactor. Typically, lithium blankets are used outside the inner walls of fusion reactors for 
the purpose of breeding tritium. With the reactor arranged in such a fashion, the D-T reactions can become self-sustaining. The D-T reaction creates a high energy neutron, which reacts with the lithium to create more tritium. To count for neutron loss, specific materials can be used to amplify neutron production. Long duration pulses are then feasible, and the idea of commercial fusion power can be realized.

Of the many types of magnetic confinement devices implemented over the last several decades, the most commonly referred to are mirror machines, stellarators and tokamaks.

A mirror reactor is a long, cylindrical magnetic confinement device. A varying magnetic field is created along the cylinder, weak in the middle, and strong on the end points. A mirror effect causes many of the charged particles to be reflected by the strong magnetic field. This is also evident in Earth's magnetosphere, where particles are trapped and oscillate between the poles. Due to collisions and scattering, however, mirror devices have proved to be impractical to be considered for steady state operation. They are, however, being highly considered as a means for space propulsion [6].

Stellarators are another common plasma confinement scheme. They implement only magnetic fields external to the plasma. No internal plasma current is needed for confinement. This makes the device less prone to instabilities associated with plasma current. The device itself is very complex in design and construction, which is the main reason why development and implementation of stellarators is not very sought after. Experiments are still being carried out in Japan, at the Large Helical Device $[7,8]$, and in Spain at the TJII $[9,10]$. The Wendelstein $7-\mathrm{X}$ is currently being constructed in Germany. An artist's representation of the Wendelstein VII is provided in Figure 1.2 [11].

The leading candidate for a magnetically confined plasma fusion device is the tokamak fusion reactor. 'Tokamak' is derived from the Russian Тороидальная камера в магнитных катушках meaning toroidal chamber in magnetic coils. The device consists of a toroidal vacuum chamber surrounded by ohmic heating coils, toroidal field coils and vertical field coils which respectively drive current, induce 


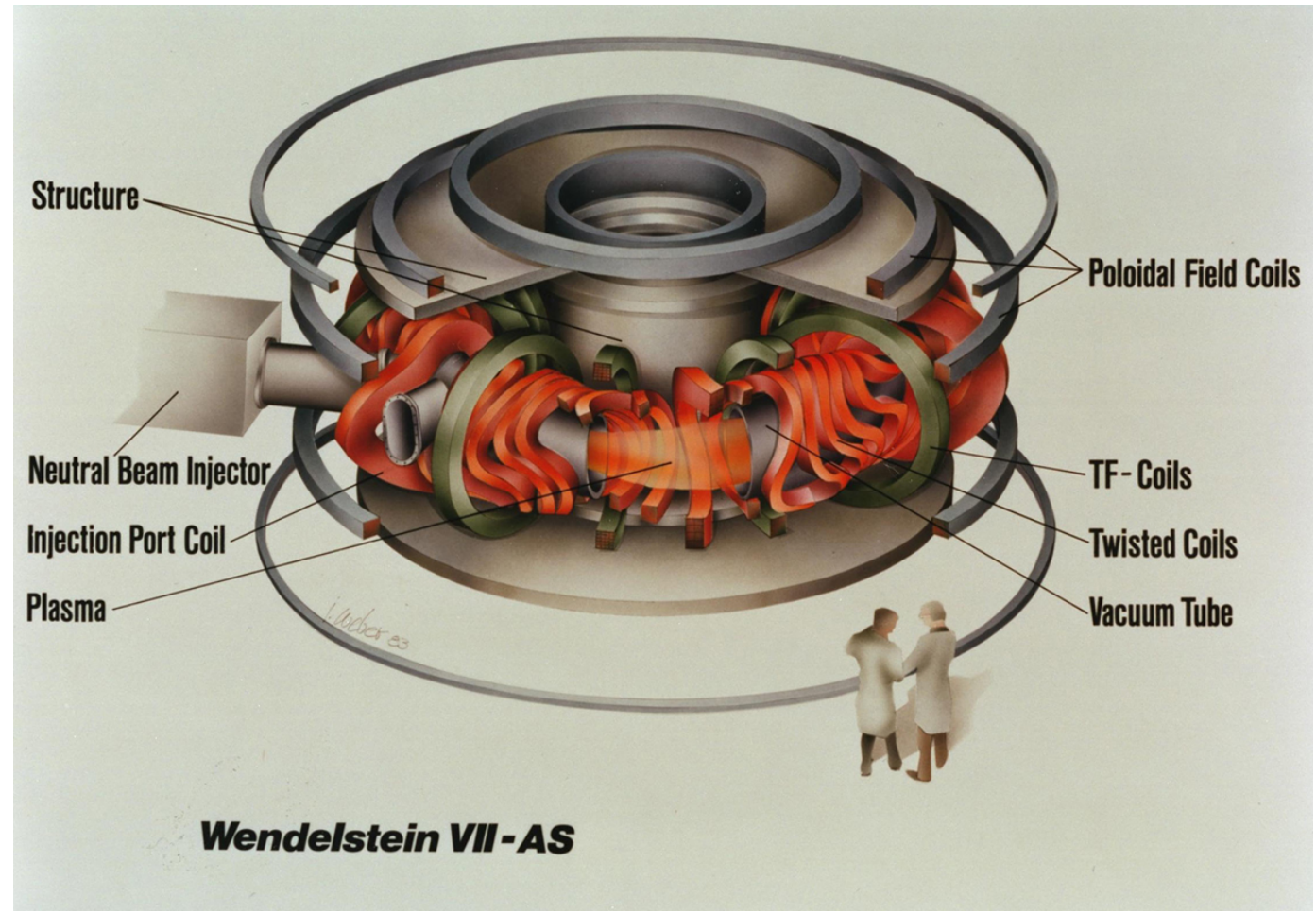

Figure 1.2: An artist's representation of the future Wendelstein VII stellarator. 
magnetic fields for confinement and control the position of the plasma column. Although more modern variations do not, some configurations also involve an iron transformer core. Figure 1.3 [11] shows a general diagram of a tokamak plasma device.

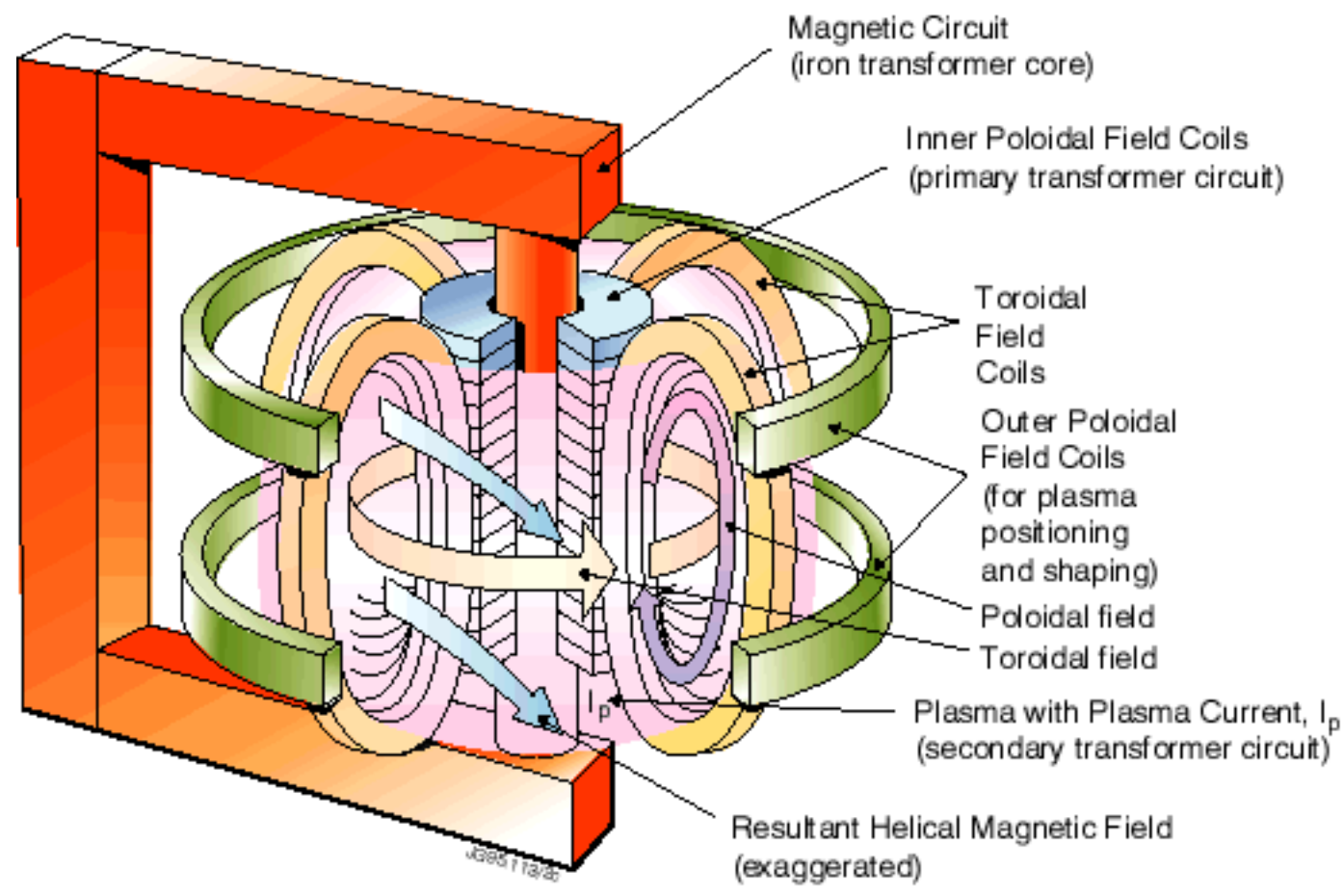

Figure 1.3: A general diagram of a tokamak showing the poloidal and toroidal magnetic coils and the resultant magnetic fields as well as the iron core transformer. Plasma current, $I_{p}$ flows in the counter-clockwise direction (from a top view).

Breakdown in a tokamak reactor is typically achieved, initially, through the introduction of seed electrons into the neutral gas along with a high voltage potential difference. Further breakdown of gas as well as maintenance of created plasma is caused through plasma heating. Ohmic, Radio Frequency (RF) waves and neutral beam injection are common methods used for plasma heating.

Ohmic heating is created through the application of a current through the plasma. Because plasma is a conductor, any current passed through it will lead to heating. This toroidal current creates the poloidal field in the plasma. RF heating is implemented by launching high frequency electromagnetic waves into the plasma. These 
waves transfer energy to the plasma, causing oscillations and collisions, which leads to further heating. In neutral beam injection, energetic neutral particles are injected into the tokamak plasma at very high velocity. The subsequent collisions with tokamak plasma particles serve to heat the plasma.

Figure 1.4 [12] shows a cross section of the Saskatchewan Torus Modified (STORM) tokamak. The inner vacuum vessel is surrounded by 16 toroidal magnetic field coils, which induce a toroidal magnetic field, confining the plasma. Their magnetic field is stronger towards the center of the tokamak. The main parameters of the STOR-M tokamak are listed in Table 1.1.

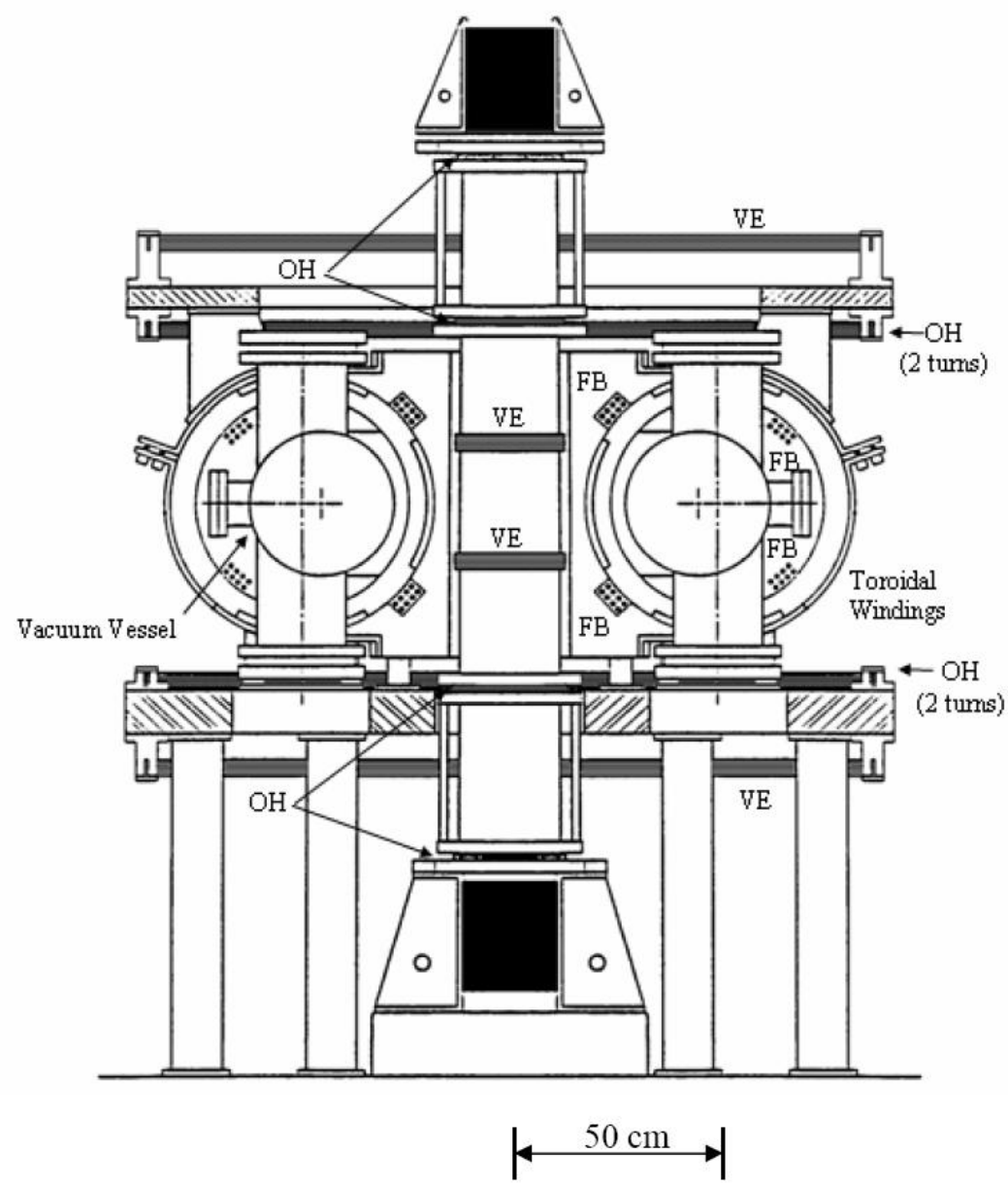

Figure 1.4: Schematic of the STOR-M tokamak, showing Ohmic coils $\mathrm{OH}$, vertical equilibrium coils VE, toroidal coils and feedback coils FB. 
Table 1.1: Parameters of the STOR-M tokamak.

\begin{tabular}{|c|c|}
\hline Parameter & Value \\
\hline Major Radius $(R)$ & $46 \mathrm{~cm}$ \\
Minor Radius $(a)$ & $12 \mathrm{~cm}$ \\
Density $(n)$ & $10^{18}-10^{19} \mathrm{~m}^{-3}$ \\
Electron Temperature $\left(T_{e}\right)$ & $\geq 100 \mathrm{eV}$ \\
Toroidal Magnetic Field $\left(B_{t}\right)$ & $\sim 1 \mathrm{~T}$ \\
Plasma Current $\left(I_{p}\right)$ & $30-50 \mathrm{kA}$ \\
Discharge Duration & $\leq 50 \mathrm{~ms}$ \\
\hline
\end{tabular}

\subsection{Tokamak Fueling and the Compact Toroid In- jector}

Unlike traditional fission reactors, where a large quantity of uranium fuel is placed in the reactor at once and power output is regulated by neutron flux, adjusted via control rods, tokamak fusion reactors use only a comparatively small amount of fuel at any given moment. It is therefore necessary to use some method of fuel replenishment to keep the reactor running. This gives the added bonus that in the unlikely event of an accident, fuel input can be stopped and a catastrophe can be easily avoided. Current fueling methods include gas injection and mechanical injection of frozen hydrogen pellets.

The earliest and simplest method of plasma fueling is gas injection. Gas valves are attached to the exterior wall of a plasma reactor (such as a tokamak) and are used to inject neutral gas into the outer portion of the pre-existing plasma. In a fusion producing reactor, a neutral D-T mixture would be injected. Energy from the pre-existing plasma is used to breakdown the newly introduced gas. Once the gas is ionized, it becomes difficult for it to move across the magnetic field and reach the core region of the reactor. Only a small fraction of the injected gas reaches the core and the rest will remain in the outer region and be pumped out for recylcing. 
For small scale tokamaks, this is often an adequate method for fuel replenishment, the simplicity and 'off-the-shelf' nature of gas valves make it ideal. The larger the tokamak, however, the harder it becomes for the fuel to reach the core of the tokamak plasma. The core of a tokamak plasma has the highest temperature and density. For fusion grade reactors, therefore, it is the central core region where most fusion processes will take place. It is imperative that the fuel reaches this location.

A relatively recent, commonly used method which marginally solves the issue of central core fueling is cryogenic pellet injection. In this case, a small, accelerated, frozen D-T pellet is mechanically fired into the tokamak plasma at high velocity. In essence, because the frozen $\mathrm{D}-\mathrm{T}$ is solid, evaporation and breakdown take longer to occur, and a significant fraction of the newly introduced fuel becomes fully ionized only once it reaches the core of the plasma.

To reach the central core of newer, very large scale tokamaks, such as the International Thermonuclear Experimental Reactor (ITER), such cryogenic pellets would require very high velocities $(\geq 3 \mathrm{~km} / \mathrm{s})$; their current speeds are limited to $\sim 0.3$ $\mathrm{km} / \mathrm{s}$ [13]. Also, because a large amount of fuel is injected in a small area, pellet injection in tokamaks has also shown to create unacceptably large density perturbations, approaching 50\%. In order to fire solid hydrogen, the pellets must be created using ultra low temperature devices, which can be inefficient and impractical.

Compact toroid injection technology is being developed to address these issues of tokamak fueling. The compact toroid injector, a coaxial plasma accelerator, shown in Figure 1.5, has the potential to efficiently fuel a tokamak by injecting plasma directly into the reactor center $[12,14,15]$ and is showing promise. Active research has been carried out in Canada, both at the University of Saskatchewan $[16,17]$ and at the former Tokamak de Varrennes (TdeV) [18,19] as well as at UC Davis [20,21], and the University of Hyogo [22, 23].

The CT, originally proposed as a fueling source for tokamaks by Parks [24] and Perkins et al. [25], is a compact, high density plasma with embedded magnetic fields. It is formed and accelerated using a coaxial magnetized plasma gun. Benefits of CT injection are that it is able to penetrate into the core region of the tokamak 
plasma as it is an excellent conductor with a high velocity and is able to overcome the tokamak's toroidal field gradient. Also, via tangential injection, CT injection provides long interaction time with tokamak plasma, and transfers momentum to the tokamak plasma, causing smaller disturbances in the discharge and inducing and sustaining toroidal rotation $[26,27]$.

The first successful demonstration of tokamak fueling through CT injection was performed on TdeV $[18,28]$ and showed that the tokamaks' particle inventory was increased without changes in the tokamak loop voltage or plasma current. As well, and possibly most important, it was shown that the tokamak was fueled with little to no increase in magnetohydrodynamic instabilities as well as an increased confinement time.

Furthermore, because the CT is electrically formed and accelerated with only a few moving mechanical parts, the process is highly repeatable, and it is able to be fired repetitively, which is important for steady state operation of the next generation tokamaks, such as the ITER tokamak [14,20,29].

Figure 1.5, [26] shows a schematic diagram of the University of Saskatchewan Compact Toroid Injector (USCTI). Table 1.2 lists the main parameters of the CT of USCTI as it travels along the acceleration region. USCTI was originally installed on the STOR-M tokamak in 1995 to study the feasibility of using CT injection to fuel tokamak reactors.

Early work involved the study of tangential injection [17], improving the fueling parameters by allowing for a larger CT-tokamak plasma interaction volume. Since then, it has been used for multiple experiments, including the study of vertical injection $[12,30]$, meant to sidestep the CT from being repelled from the tokamak toroidal field gradient, as well as improved confinement modes [26]. 


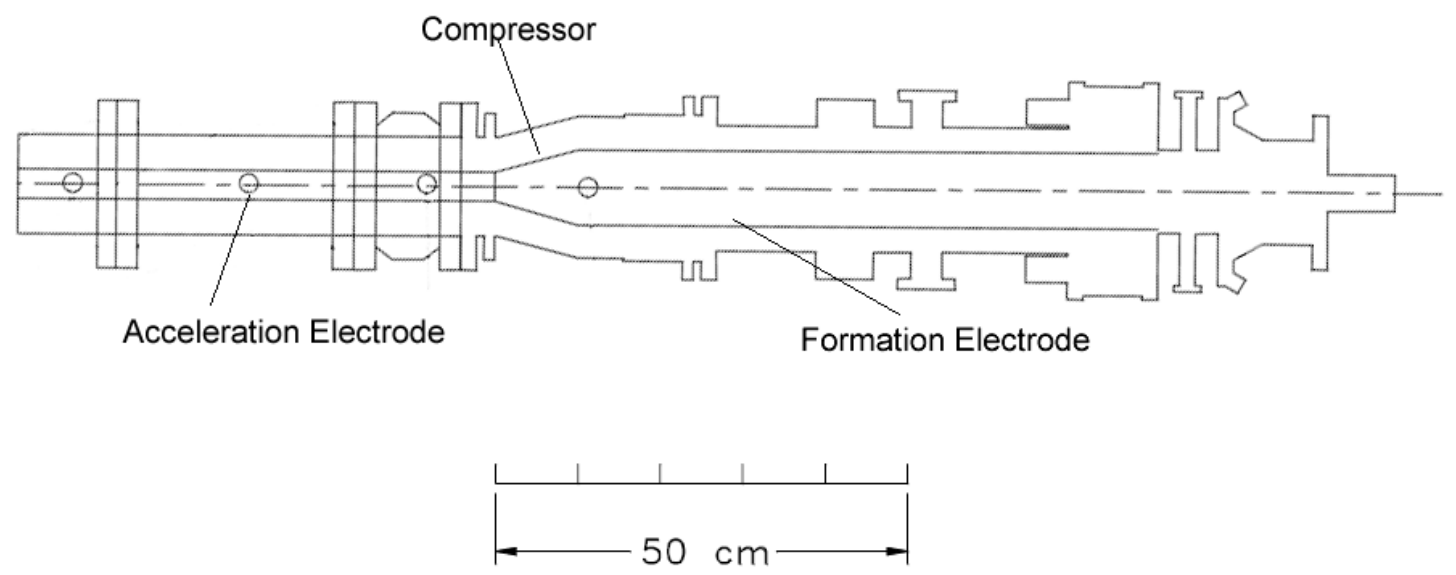

Figure 1.5: General schematic of a CT injector, showing formation, compression and acceleration regions.

Table 1.2: Parameters of CT at exit of accelerator.

\begin{tabular}{|c|c|}
\hline Parameter & Value \\
\hline Radius & $5 \mathrm{~cm}$ \\
Length & $\sim 15 \mathrm{~cm}$ \\
Density & $1-4 \times 10^{21} \mathrm{~m}^{-3}$ \\
Electron temperature & $\leq 10 \mathrm{eV}$ \\
Magnetic Field & $\sim 0.2 \mathrm{~T}$ \\
Velocity & $\geq 200 \mathrm{~km} / \mathrm{s}$ \\
Mass & $\leq 1 \mu \mathrm{g}$ \\
\hline
\end{tabular}




\subsection{Motivation for Repetitive Compact Torus Op- eration}

For fusion devices to be feasible for providing energy, they must be run in a steady state, or long pulse, mode. The targeted duration for ITER plasma is on the order of 400 seconds. For a commercial application, a tokamak would need to be run for much longer durations. Because tokamaks contain only a small amount of fuel at any given moment, as described in Section 1.3, it is clear that whatever fueling method is used, it must be able to continuously fuel the reactor.

Because a CT injector fires quantized amounts of plasma, and cannot be fired in a continuous jet, for application in modern, large scale tokamaks, it must be implemented in a repetitive mode. The rate of fueling is determined by the amount of fuel needed per unit time, the density perturbation allowable in order to maintain the tokamak plasma and the size and density of CT that can be created. Optimal frequency for CT injection in conjunction with ITER experiments has been estimated to be $f \sim 50 \mathrm{~Hz}$, calculated based on a desired fueling rate for ITER of $90 \mathrm{~Pa} \cdot \mathrm{m}^{3} \cdot \mathrm{s}^{-1}$ using a $1.29 \mathrm{mg}$ CT [31], although slightly lower estimations, $f \sim 20 \mathrm{~Hz}$, have been made [29].

Experiments, primarily at Lawrence Livermore National Laboratory's Ring Accelerator Experiment (RACE), have been performed with CTs of large masses (500 $\mu \mathrm{g}$ ) as well as high velocities (up to $2000 \mathrm{~km} / \mathrm{s}$ [32]) but despite the obvious advantages to $\mathrm{CT}$ fueling, and the necessity of repetitive implementation, little work has been done to further the technology. The only previous work on repetitive CT firing for tokamak fueling has been performed on the Compact Torus Injection Experiment (CTIX) at UC Davis $[20,33]$. The device uses the passive breakdown of the CT plasma as the switch for formation and acceleration bank firing and the necessary delay between the formation and acceleration discharges is realized by using saturable cores [34]. With this setup, it is possible to fire 1000 successive shots at a maximum frequency of $0.2 \mathrm{~Hz}$. Their main limitation on frequency and length 
of firing was heating of the solenoid in the inner formation electrode and a limited pumping rate [33].

At Nihon University, Shimamura et al. [35,36] use the current waveform of their discharging capacitors to create multiple CTs in high repetition. This takes advantage of the decaying waveform generated by a discharging capacitor to create a short pulse train of CTs. Their primary experiment is to study the effects of accumulation in CT-CT collisions [37]. Although high repetition $(100 \mathrm{kHz})$ is achieved, their experimental setup is not sustainable, as the pulse train only lasts for a short amount of time ( 3-4 shots) at which point their capacitors must be recharged.

No previous work has been performed on repetitive firing of USCTI. The purpose of this research was to study the feasibility and reproducibility of implementing USCTI in a repetitive mode. This improves upon experiments on CTIX as USCTI was tested at higher frequencies. Furthermore, it improves upon experiments at Nihon University, as USCTI was tested in a higher repeatability mode (i.e. with larger numbers of sequential shots). The primary goal of this thesis was to upgrade the charging power supply and to study the feasibility of firing USCTI at a frequency greater than $0.2 \mathrm{~Hz}$.

Showing feasibility of this increased repetitiveness is the next step of CT injection. It brings the realm of $\mathrm{CT}$ injection to the next step, bringing it closer to integration with large scale tokamaks on a practical level.

\subsection{Thesis Outline}

This thesis has been divided into six Chapters. This first Chapter dealt with a general introduction to fusion power, the fusion reactions of interest, fusion reactors and more specifically the tokamak reactor. Also discussed were, currently used fueling methods, the compact torus injector, and the need for repetitive fueling. The second Chapter delves into the physics behind the CT, including descriptions of its formation, acceleration and integration with tokamak plasmas. An explanation and description of USCTI, including modifications made to facilitate repetitive operation, 
is given in Chapter three. Chapter four discusses the diagnostics, data acquisition and post processing techniques used. In the fifth Chapter, the experimental results are presented, demonstrating the feasibility of repetitive operation. The sixth and final Chapter presents a summary of the results, as well as suggestions for future work. 


\section{CHAPTER 2 \\ COMPact Torus Physics}

This chapter discusses the physics behind compact toroids, the basic concepts, structure and implementation in acceleration experiments. Firstly, a description of the concept behind CTs is given, followed by a discussion of their magnetic structure. Thirdly, a description of their formation and acceleration is provided. Finally, their integration with tokamaks is discussed.

\subsection{Basic Concept}

The term 'spheromak' is used synonymously with 'compact torus' in literature as even though their respective shapes are slightly different, their other properties are alike. Spheromaks without central holes are sometimes referred to as spherical spheromaks, whereas CTs (which have a central hole) are sometimes referred to as cylindrical spheromaks [34].

Spheromaks are often discussed in astrophysics, as they are seen as a natural form of plasma evolution. Simply put, they are a toroidal, self-sustained plasma. That is, other than initial formation, no external currents or magnetic fields are needed; the magnetic fields are formed via currents from within the plasma itself. Three criterion for a spheromak configuration are [34]:

1. a simply connected bounding surface, i.e. contained in a spheroidal chamber,

2. both toroidal and poloidal magnetic fields,

3. closed poloidal flux surfaces. 
Because the spheromak is not linked by any external magnetic fields, the toroidal magnetic field vanishes at the bounding surface. This is a key aspect, separating spheromaks from other toroidal confinement schemes, such as tokamaks.

CTs are typically described as a low $\beta$ plasma, where $\beta$ is defined as

$$
\beta=\frac{2 \mu_{0} P}{B^{2}}
$$

with $B$, the magnetic field, $P$ the thermal pressure, and $\mu_{0}$ the permeability of free space. Typically, $\beta \sim 0.01-0.2$ for spheromak plasmas [34]. This implies that the forces involved in spheromaks are predominantly magnetic, and to a first order approximation, thermal pressures can be ignored. They are also typically a low aspect ratio plasma, $a \simeq R$, meaning that the minor radius is about equal to the major radius and the plasma is essentially a sphere; in an ideal spheromak $a=R$. Because CTs are formed in a coaxial gun (propelled by the $\vec{J} \times \vec{B}$ force) they typically have $a<R$ while in the injector portion. As they are compressed and injected into the tokamak, their major radius is decreased, becoming much more similar in shape to a spheromak.

\subsection{Magnetic Structure of the Compact Torus}

The CT relaxes into a force-free Taylor state after formation. In this configuration $\mathbf{J} \| \mathbf{B}$ and $\mathbf{J} \times \mathbf{B}=0$, so Ampere's law reduces to

$$
\nabla \times \mathbf{B}=\mu_{0} \mathbf{J}=\lambda \mathbf{B}
$$

with $\lambda$ in units of inverse length and constant in a force free state. Figure 2.1, from Geddes [38] shows an axially symmetric CT plasma surrounded by a cylindrical flux conserver. With boundary conditions of $B_{r}, B_{\phi}=0$ and $r=R_{f c}$ solutions for the poloidal $\left(B_{r}, B_{z}\right)$ and toroidal $\left(B_{\phi}\right)$ magnetic fields can be found by first expanding Equation 2.2 in cylindrical coordinates as 


$$
\begin{aligned}
\frac{1}{r} \frac{\partial B_{z}}{\partial \phi}-\frac{\partial B_{\phi}}{\partial z} & =\lambda B_{r} \\
\frac{\partial B_{r}}{\partial z}-\frac{\partial B_{z}}{\partial r} & =\lambda B_{\phi}, \\
\frac{1}{r}\left[\frac{\partial\left(r B_{\phi}\right)}{\partial r}-\frac{\partial B_{r}}{\partial \phi}\right] & =\lambda B_{z} .
\end{aligned}
$$

The magnetic field has an azimuthal, $\hat{\phi}$, periodic variation, and so

$$
\mathbf{B}(r, \phi, z)=\mathbf{B}(r, z) e^{-i m \phi},
$$

where $m$ is an integer. This leads to, by letting $\partial / \partial z \rightarrow i k_{z}$ through a $z$ coordinate Fourier transform and $\partial / \partial \phi \rightarrow-i m$ from the periodic nature of $\hat{\phi}$,

$$
\begin{aligned}
B_{r} & =\frac{i m \lambda B_{z}}{r k_{r}{ }^{2}}-\frac{i k_{z}}{k_{r}^{2}} \frac{\partial B_{z}}{\partial r} \\
B_{\phi} & =\frac{m k_{z} B_{r}}{k_{r}^{2} r}-\frac{\lambda}{k_{r}^{2}} \frac{\partial B_{z}}{\partial r} \\
0 & =\frac{\partial^{2} B_{z}}{\partial r^{2}}+\frac{\partial B_{z}}{r \partial r}+\left(k_{r}^{2}-\frac{m^{2}}{r^{2}}\right) B_{z}
\end{aligned}
$$

where $k_{r}^{2}=\lambda^{2}-k_{z}^{2}$ and Equation 2.9 is Bessel's equation, with the solution $B_{z}=$ $B_{0} J_{m}\left(k_{r} r\right)$ (assuming $k_{r}^{2} \neq 0$ ). This can then be rewritten, in the case of $m=0$, as

$$
\begin{aligned}
B_{r} & =-B_{0} \frac{k_{z}}{k_{r}} J_{1}\left(k_{r} r\right) \cos \left(k_{z} z\right) \\
B_{z} & =B_{0} J_{0}\left(k_{r} r\right) \sin \left(k_{z} z\right) \\
B_{\phi} & =B_{0} \frac{\lambda}{k_{r}} J_{1}\left(k_{r} r\right) \sin \left(k_{z} z\right) .
\end{aligned}
$$

$J_{0}$ and $J_{1}$ are Bessel functions of the first kind [38,39]. In the case of Figure 2.1, $k_{z}=\pi / L_{f c}$ and $k_{r}=3.83 / R_{f c}$ are the axial and radial wavenumbers where $L_{f c}$ and $R_{f c}$ are the length and radius, respectively, of the flux conserver.

The magnetic axis can be found via Equation 2.10, by letting $B_{z}=0$ and solving the bessel function, as $r=0.63 R_{f c}$. This becomes functionally important for diagnostics, further discussed in Section 4.1.4. 


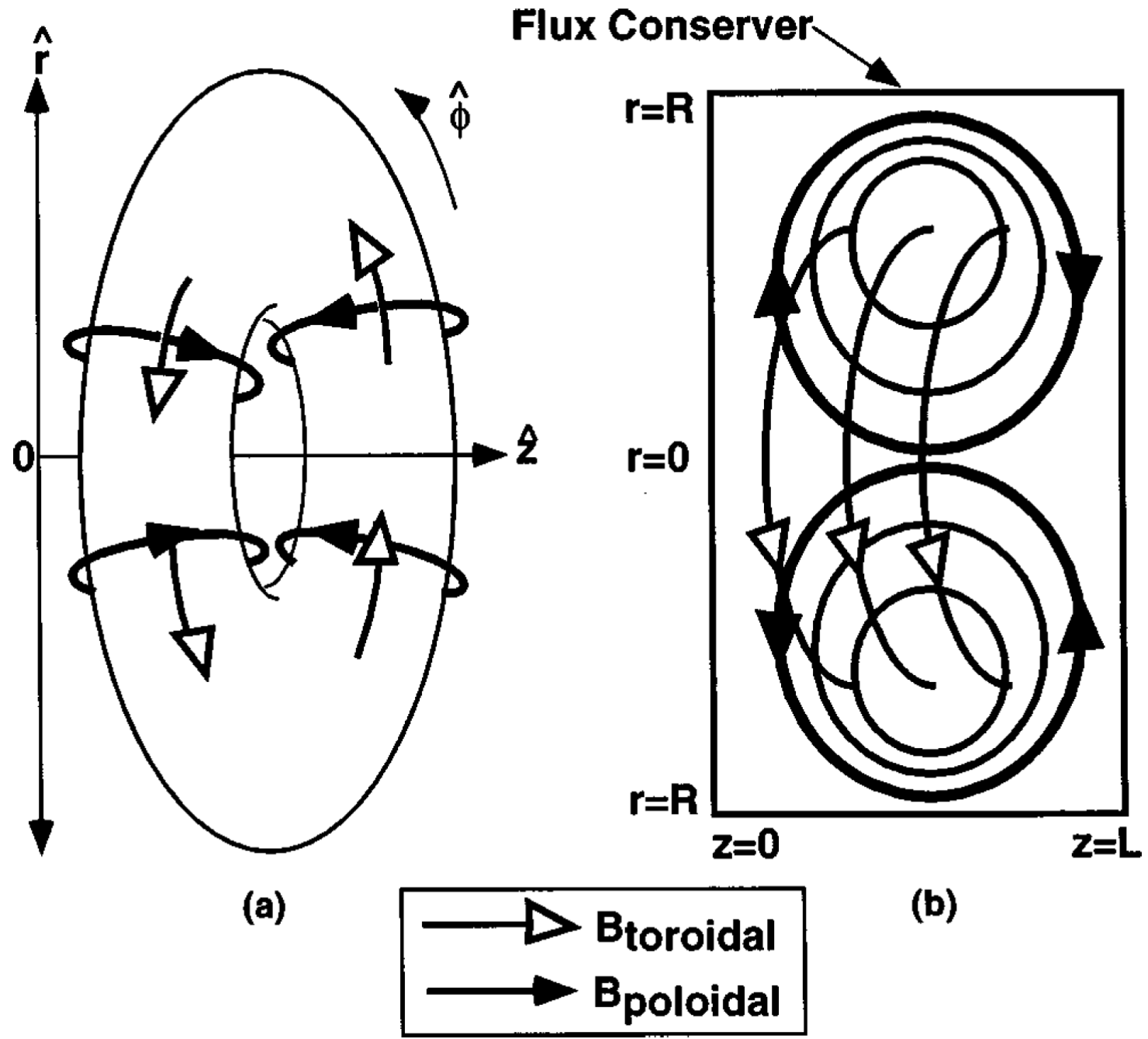

Figure 2.1: Diagram of CT magnetic fields. In the case of USCTI, the inner acceleration electrode is located along the $\hat{z}$ axis. 


\subsection{Formation and Acceleration}

The bulk of the energy in a USCTI discharge is provided through discharging two $20 \mu F, 25 \mathrm{kV}$ capacitor banks, one for CT formation, and another for acceleration. Most CT accelerators operate in a similar fashion. The formation bank's energy is harnessed to breakdown the gas and create a plasma. The acceleration bank

discharge is used to create a Lorentz force $(\vec{J} \times \vec{B})$ which constantly accelerates the plasma through the accelerator.

\subsubsection{Formation}

The three main components of the formation section are the solenoid, the gas valves and the formation electrodes. As shown in Figure 2.2, the first process is the firing of the solenoid capacitor bank, which creates a quasi steady-state magnetic flux. The solenoid bank is fired well in advance to allow time for the magnetic field of the solenoid to penetrate the inner formation electrode. Immediately afterwards, the fast acting gas valves are pulsed using a separate capacitor bank, sending pure hydrogen gas into the formation region. The formation capacitor bank, with a voltage exceeding the breakdown threshold for the hydrogen contained between the electrodes, is then discharged, sending a current across the formation electrodes, breaking down the gas and creating a toroidal field in the CT. The CT is pushed across the magnetic flux region of the solenoid, which turns the solenoid field into the poloidal magnetic field of the CT after the field lines reconnect at the trailing edge of the CT.

\subsubsection{Acceleration}

The CT is in a minimum magnetic energy state, a result of its self-organization. The poloidal and toroidal magnetic field structure makes the CT very robust, allowing it to remain intact even if the entire system is accelerated. Furthermore, due to the high conductivity of the copper electrodes, the CT is not physically linked to any external structure and the image current on the electrode preserves the magnetic 


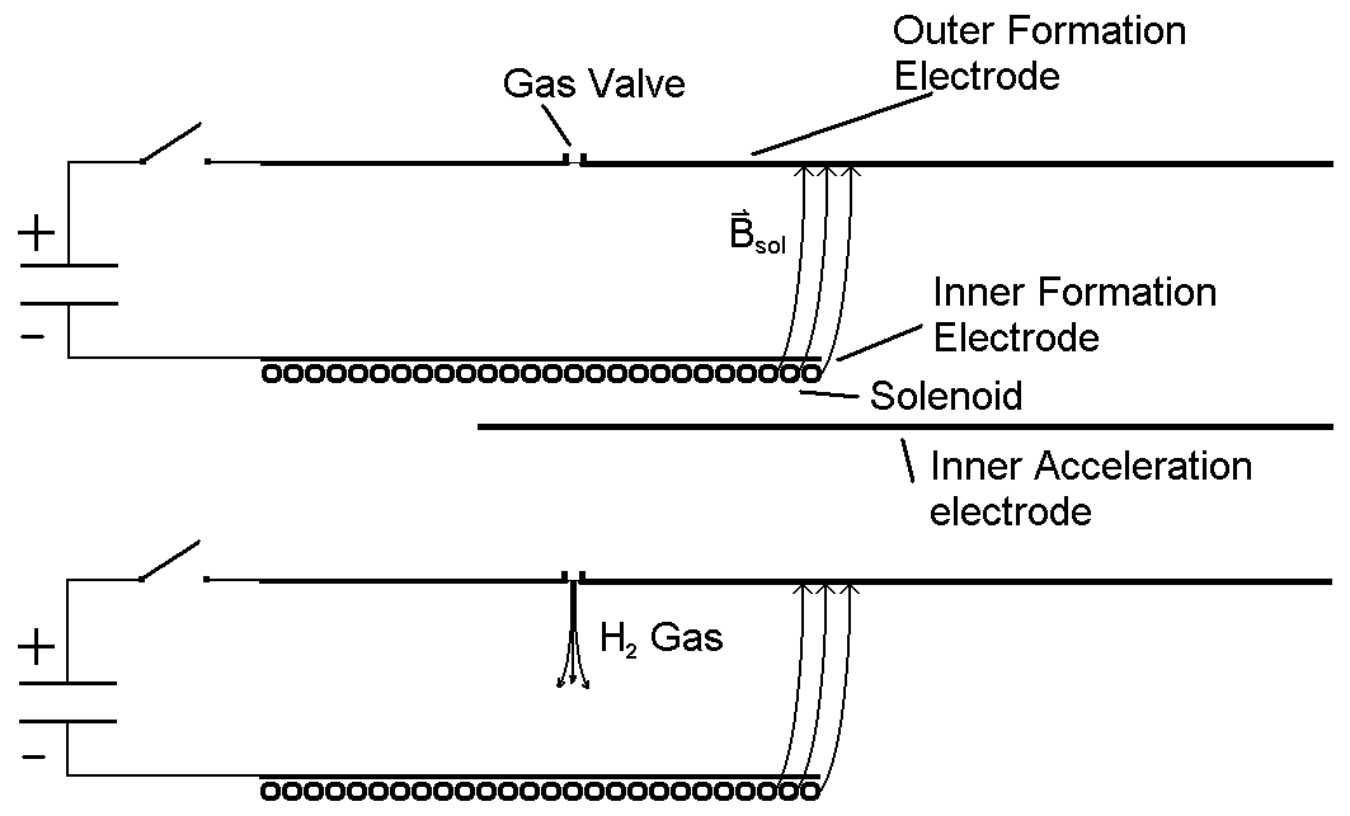

a)

b)

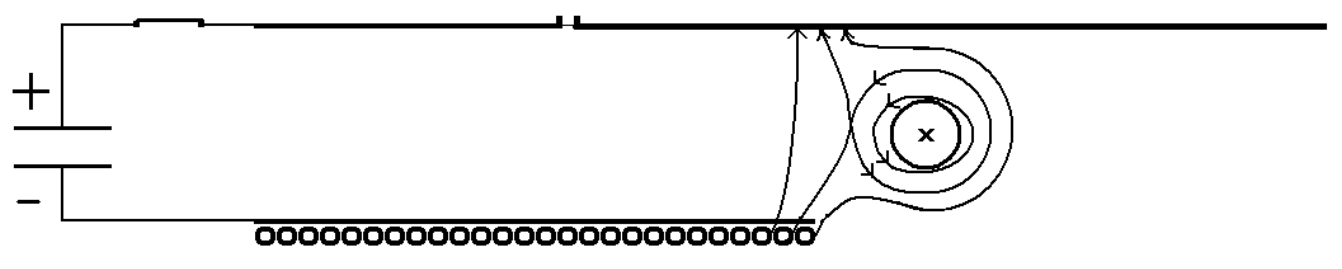

c)

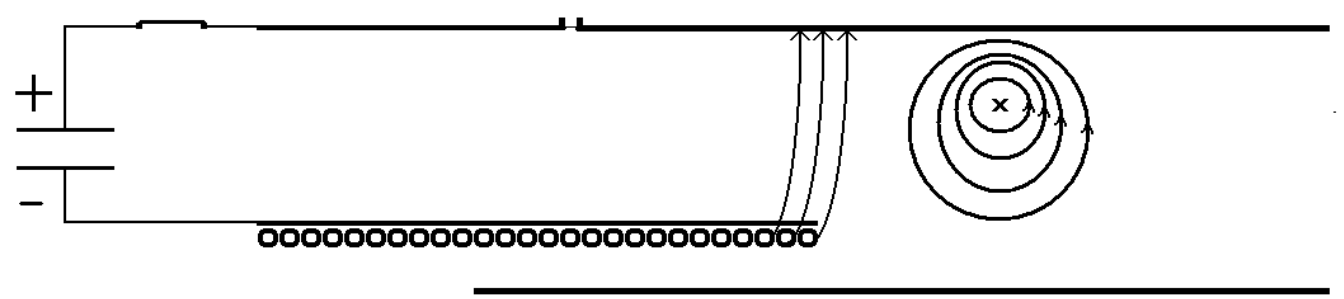

d)

Figure 2.2: A typical formation section for a CT accelerator, a) the solenoid is energized, b) gas is injected in the formation section, c) the formation bank is discharged, breaking down the gas, d) after the CT is pushed through the solenoid field, a poloidal field remains in the CT. 
field in the CT. When traveling through the acceleration region, the CT merely acts as a sliding short. When the acceleration capacitor bank is fired, current flows from the bank and through the inner acceleration electrode. Just as in the formation process, it then passes through the CT plasma and out to ground through the outer acceleration electrode. The radial current component, $\vec{J}$, passing through the $\mathrm{CT}$, interacts with the toroidal field, $\vec{B}_{\theta}$, present in the CT plasma to produce the Lorentz force

$$
\vec{F}=\vec{J} \times \vec{B}_{\theta}
$$

which leads to an acceleration of the CT, as shown in Figure 2.3.

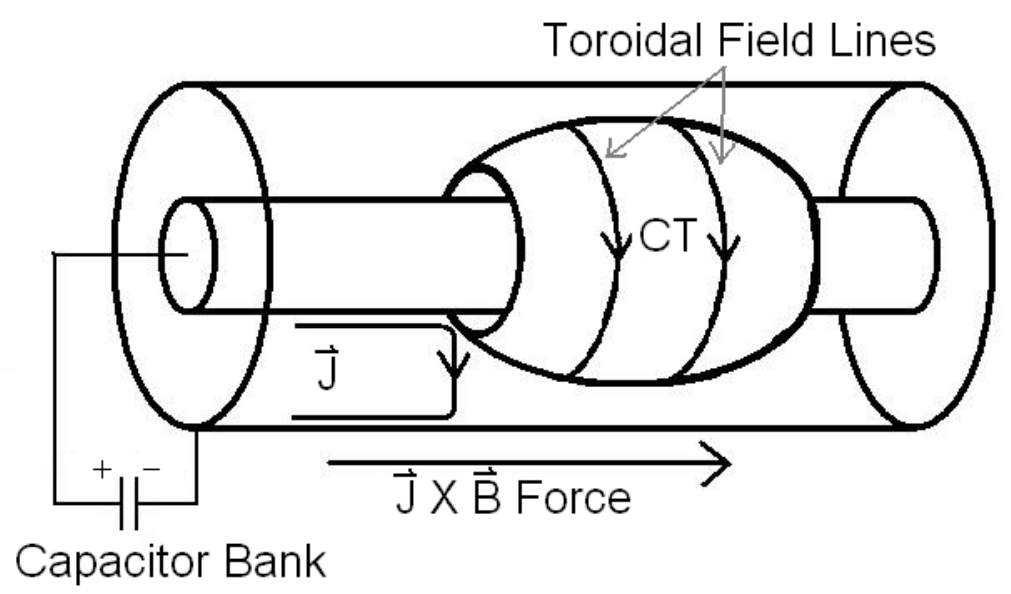

Figure 2.3: Motion of a CT along an accelerator. $\vec{B}$ is azimuthal, $\vec{J}$ is radial and $\vec{J} \times \vec{B}$ is to the right.

\subsection{Compact Torus Injection}

A CT is typically injected into a tokamak radially, from the outboard, low magnetic field side. The tokamak toroidal field is inversely proportional to the tokamak major radius, 


$$
B_{T}=\frac{\mu_{0} N I}{2 \pi R}
$$

where $I$ is the current through a toroidal magnetic field coil winding, $N$ the total number of windings, and $R$ the distance from the center of the tokamak. There exists a magnetic field gradient increasing towards the center of the tokamak. The spatially increasing magnetic field must be displaced by the CT (modeled as a hard conducting sphere), causing the CT's kinetic energy to decrease. If the tokamak's toroidal field is large enough, the CT will be stopped at the center of the tokamak, if it is too large, the CT will not penetrate into the core-region of the tokamak plasma. Therefore the basic requirement for core fueling is that the translational kinetic energy density of the CT must be larger than the magnetic field energy density of the tokamak [30],

$$
\frac{1}{2} \rho_{C T} v_{C T}^{2}>\frac{B_{T}^{2}}{2 \mu_{0}}
$$

where $\rho_{C T}$ is the mass density of the CT plasma, $v_{C T}$ is the CT velocity as it enters the tokamak and $B_{T}$ is the tokamak's magnetic field strength at its center. Also, the CT plasma density is normally much greater than the tokamak plasma density and $v_{C T}$ is lower than the Alfvén velocity in the tokamak discharge.

The Alfvén velocity is the characteristic velocity of the Alfvén wave. This is a hydromagnetic wave of low-frequency ion oscillations traveling along the principal magnetic field of a plasma. The Alfvén velocity, $v_{A}$, for a plasma is given by the magnetic field, $B$, and mass density, $\rho$, as [40]

$$
v_{A}=\frac{B}{\sqrt{\mu_{0} \rho}} .
$$

For STOR-M, $v_{A} \sim 7000 \mathrm{~km} / \mathrm{s}$ and $v_{C T} \sim 200 \mathrm{~km} / \mathrm{s}$ for the USCTI injector.

\subsubsection{Compact Torus Lifetime}

Ideally, the CT would last forever, but due to a finite, yet minuscule, resistivity in the plasma, the CT lifetime is limited by the decay of the internal magnetic fields. The time for a magnetic field to diffuse through a distance $l$ in a plasma is [12] 


$$
\tau_{C T}=\mu_{0} \frac{l^{2}}{\eta},
$$

where $\eta$ is the Spitzer resistivity of the plasma, given in $\Omega \cdot \mathrm{m}$ by

$$
\eta=1.65 \times 10^{-9} Z_{e f f} \frac{\ln \Lambda}{T_{e}^{\frac{3}{2}}},
$$

with $T_{e}$ in $\mathrm{keV}, Z_{\text {eff }}$ the effective charge and $\ln \Lambda$ the Coulomb logarithm. Replacing $l$ by assuming $l \sim r_{C T} / 5$, with $r_{C T}$ the $\mathrm{CT}$ radius, we have [25],

$$
\tau_{\text {life }}=30.5 r_{C T}^{2} \frac{T_{e}^{\frac{3}{2}}}{Z_{\text {eff }} \ln \Lambda}
$$

where

$$
\ln \Lambda \approx 31-\ln \frac{\sqrt{n_{C T}}}{T_{e}} .
$$

Therefore the larger the CT and the higher the temperature, the longer its lifetime. For USCTI, with $n_{e} \sim 10^{21} \mathrm{~m}^{3}$ and $T_{e} \sim 10 \mathrm{eV}$ giving an approximate value for the CT lifetime of $\tau_{\text {life }}=20 \mu \mathrm{s}$.

\subsubsection{Tokamak Penetration}

To ensure that the CT structure remains intact during penetration, the CT lifetime must be longer than the penetration time, $\tau_{p}$,

$$
\tau_{\text {life }} \gg \tau_{p}
$$

$\tau_{p}$ can be described as the time it takes for the CT to propagate to its stopping point,

$$
\tau_{p} \approx \frac{L_{p}}{v_{C T}}
$$

where $L_{p}$ is the distance between the CT muzzle and the CT stopping point in the tokamak core plasma.

Penetration location, $R$, into the tokamak, can be determined by substituting

$$
B(R)=B_{0} \frac{R_{0}}{R}
$$


into Equation 2.15, where $B_{0}$ is the magnetic field at the radius, $R_{0}$, of the magnetic axis, to obtain

$$
R=B_{0} \frac{R_{0}}{v_{C T} \sqrt{\mu_{0} \rho_{C T}}} .
$$

So the penetration depth increases (i.e., decrease in radius $R$ ) with increasing CT density, and more effectively with increasing velocity.

\subsubsection{Magnetic Reconnection and Fuel Deposition}

Originally, CTs were devised to be injected simply normal to the tokamak plasma. Presently, however, CTs are injected tangentially into tokamaks. USCTI, for example, is arranged such that it is injected at an angle of $27^{\circ}$ to STOR-M.

This tangential injection provides several benefits to the deposition of fuel into the tokamak. First, the volume of interaction is maximized. This gives the CT more room (and time) with which it can deposit itself into the tokamak plasma. Secondly, the interaction is much smoother. The abrupt interaction of a normal injection may cause perturbations in the tokamak plasma. Lastly, it allows for the CT momentum to contribute to the desired tokamak plasma rotation.

As previously discussed, CT injectors provide the smoothest fuel deposition when compared to other presently used fueling techniques (gas injection and pellet injection). Partially, this is due to the fact that the CT carries more energy when entering the tokamak plasma compared to gas injection or, especially, pellet injection (as it is already a plasma). Because of this, it does cause drastic cooling of the tokamak plasma during fueling.

The main reason, however, for a CT's fuel deposition capabilities is due to its very magnetic nature. The process of magnetic connection of a CT with a tokamak plasma is demonstrated in Figure 2.4, from Perkins [25].

With a first order approximation, a CT (or spheromak) plasma acts essentially like a magnetic dipole due to its toroidal current. This fact is a central point to reactor fueling, as when the $\mathrm{CT}$ enters a tokamak, it will rotate its dipole to align 


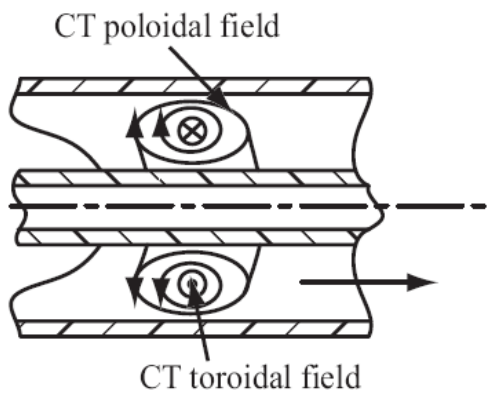

a)

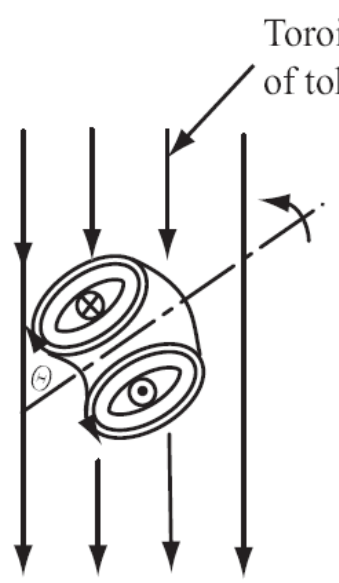

b)
Toroidal field

tokamak

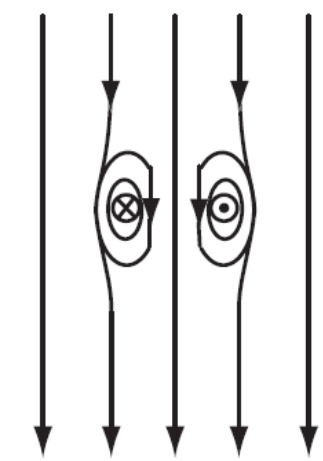

c)

Figure 2.4: The CT as it a) is accelerated through the injector b) tilts in the tokamak magnetic field and c) reconnects at the fuel deposition point

with the tokamak toroidal field. This process is not simply an act of fuel deposition, but one of magnetic connection. Once the CT has aligned its magnetic dipole with the tokamak toroidal field, the poloidal field lines in the CT plasma will connect with the toroidal field lines within the tokamak plasma. The charged particles in the CT will enter the tokamak plasma along the reconnected magnetic field lines. This physical linking, through the connected magnetic field lines, of the CT plasma to the tokamak plasma drastically aids in a smooth, (i.e. minimal perturbation) fuel deposition.

\subsection{Summary}

This chapter presented details of the physics behind compact toroids. Their basic concept was explained, as well as their magnetic structure. The process of the formation and acceleration of CTs was discussed. Finally, their implementation in tokamak fusion reactors was also described, including tokamak penetration, magnetic reconnection and fuel deposition. 


\section{Chapter 3 \\ Repetitive Operation of the USCTI IN- JECTOR}

As previously discussed, the primary objective of this project was to explore the feasibility of running a CT injector, namely USCTI, in a repetitive mode. In order to design and construct the type of repetitive CT injectors needed for future generation tokamaks, an understanding of the limitations of present devices is needed. This chapter gives a more detailed look at USCTI and provides a description of the modifications made to USCTI to facilitate rep-rated operation.

\section{$3.1 \quad$ USCTI}

USCTI is a combination of a Marshall gun [41] and a coaxial rail gun. Its main sections, shown in Figure 3.1, from Liu [12] are the formation, the compression, acceleration and the focussing cone.

\subsubsection{Basic Injector Design}

The formation region of USCTI consists of two coaxial stainless steel electrodes. The outer electrode serves as the vacuum chamber and the inner electrode surrounds the solenoid. The stainless steel, with high resistivity, is used to ensure the penetration of the solenoid magnetic field into the formation region. To prevent arcing and aid in cooling, the solenoid is physically separated from the inner electrode and surrounded by $\mathrm{SF}_{6}$ gas, pressurized to 2 bar. $\mathrm{SF}_{6}$ is commonly used both as an electrical insulator and a heat conducting medium. The solenoid induces, at its end, a radial magnetic 


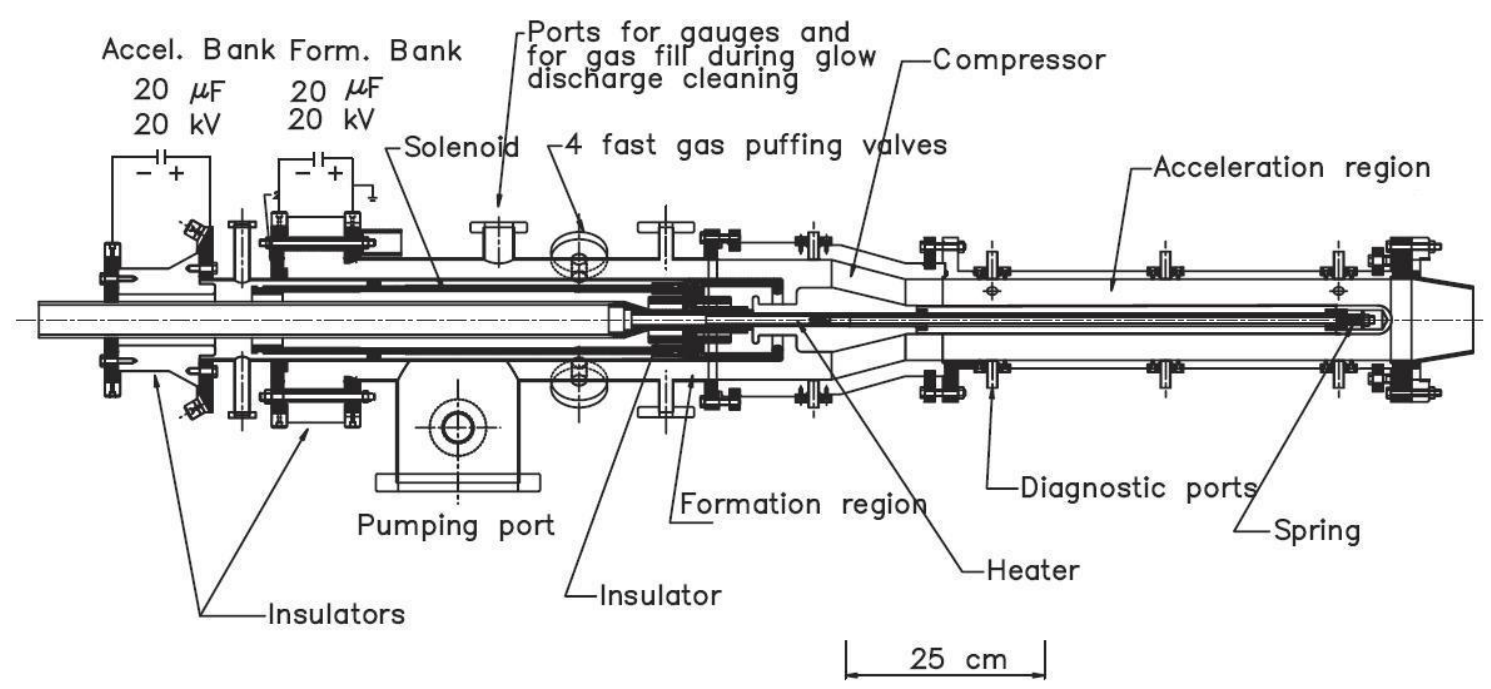

Figure 3.1: USCTI cross section, depicting locations of the gas valves, diagnostic ports as well as formation and acceleration regions.

field in the formation region, which eventually becomes the poloidal magnetic field of the CT (see Section 2.3.1).

The compression region, which follows the formation section, is made from OxygenFree High Conductive (OFHC) copper. The copper serves as a flux conserver, conserving the magnetic field of the CT during its lifetime. The CT's lifetime $(\sim 20 \mu \mathrm{s})$ is much shorter than the time it would take for the magnetic fields from the $\mathrm{CT}$ to diffuse through the copper walls $(\geq 1 \mathrm{~ms})$. This region also serves to compress the CT as well as disconnect it from the solenoid magnetic field before it passes through to the acceleration region. The compression serves to create a more compact, relaxed, spheromak-like toroid (seen in the $B_{z}$ measurements of Chapter 5). Furthermore, the sudden increase in the gap between the inner and outer electrodes before the compression region helps the $\mathrm{CT}$ balloon out and disconnect itself from the solenoid's magnetic field.

The acceleration region is a long $(64 \mathrm{~cm})$, straight section of OFHC copper with constant inner and outer radii, 1.8 and $5 \mathrm{~cm}$, respectively. Diagnostic ports are placed along the acceleration region to measure the CT parameters as it is accelerated. At 
the end of the acceleration region lies a focussing cone, which further compresses the CT in order to match the CT size with the STOR-M plasma before being injected into the tokamak plasma.

\subsubsection{Vacuum Systems and Gas Injection}

Although USCTI was operated while attached to the tokamak, it also has the benefit of being able to operate separately. Near the formation region, as shown in Figure 3.1, there is a pumping port, to which a 450 liter/second $(\mathrm{L} / \mathrm{s})$ Leybold TURBOVAC Turbo Molecular Pump (TMP) is attached. In combination with the $1000 \mathrm{~L} / \mathrm{s}$ TMP of the tokamak, the ultimate base pressure achievable is $(1 \sim 3) \times 10^{-7}$ torr. A gatevalve and a manual throttle bypass valve are used. The manual valve is meant to facilitate glow discharging between the electrodes at high pressures, but it also serves to evacuate the chamber during gas valve calibration, so that the main gate-valve is not overused.

Because USCTI is attached to the STOR-M tokamak, its operation is benefitted by the extra equipment. STOR-M uses an additional 1000 L/s Leybold TMP system. This system is highly beneficial to repetitive operation as STOR-M has a much larger volume than USCTI, the extra pump provides a remarkable increase in the ability to evacuate the chamber after each shot, which was a problem observed at CTIX [20]. In addition, the vacuum vessel of STOR-M itself acts as a large test chamber as it allows for the unused gas, at the end of the CT lifetime, to diffuse through a large volume.

Furthermore, it was found that the connection to the tokamak was, in fact, needed, as problems developed with the USCTI vacuum pump. When USCTI was fired, it was discovered that its vacuum pump inadvertently switched to its 'acceleration' mode, meaning it was as if the power on the pump was cycled, and it re-entered its warm up stage. It was originally believed to be a problem with grounding, however this was found not to be the case, as any alterations to the grounding for the vacuum pump was found to have no effect on the problem. The problem seems to be coming from noise from the electrical system, although its mitigation has not been 
achieved yet.

There are four gas valves placed azimuthally at $90^{\circ}$ intervals to provide even distribution of pure hydrogen into the USCTI formation region. These gas valves were specifically designed to have a fast reaction time. The gas valves, shown in Figure 3.2 [12], are operated by passing current through a planar coil, which then pushes open a small piston. The Full Width at Half Maximum (FWHM) discharge current for the gas valves' opening is $\sim 90 \mu \mathrm{s}$. The coils are connected in parallel to a common capacitor bank. The bank voltage controls the gas throughput. In addition, gas throughput is tunable via a screw and spring system in the valves. Turning the screw alters the force against the spring which presses against the piston, adjusting its ability to open. The valves are calibrated such that when fired without breakdown into the tokamak vessel, they deliver an increased pressure of $2.5 \times 10^{-4}$ torr in the STOR-M/USCTI chamber. This corresponds to a particle inventory of $\sim 10^{19}$ particles.

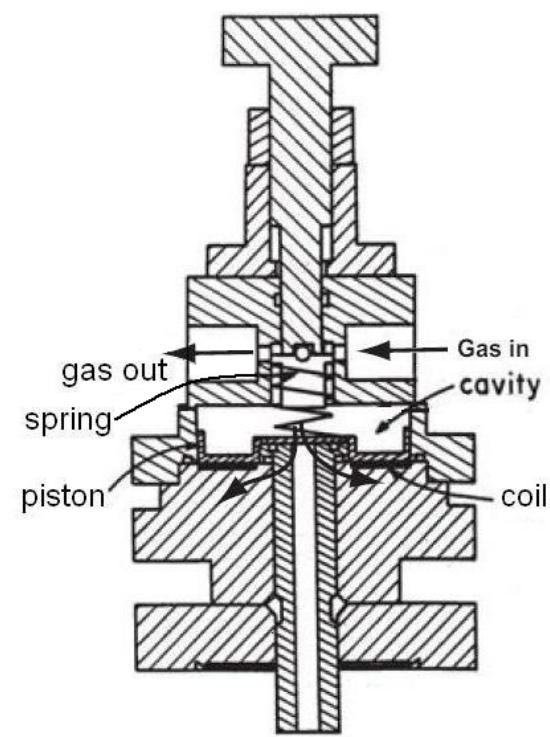

(a)

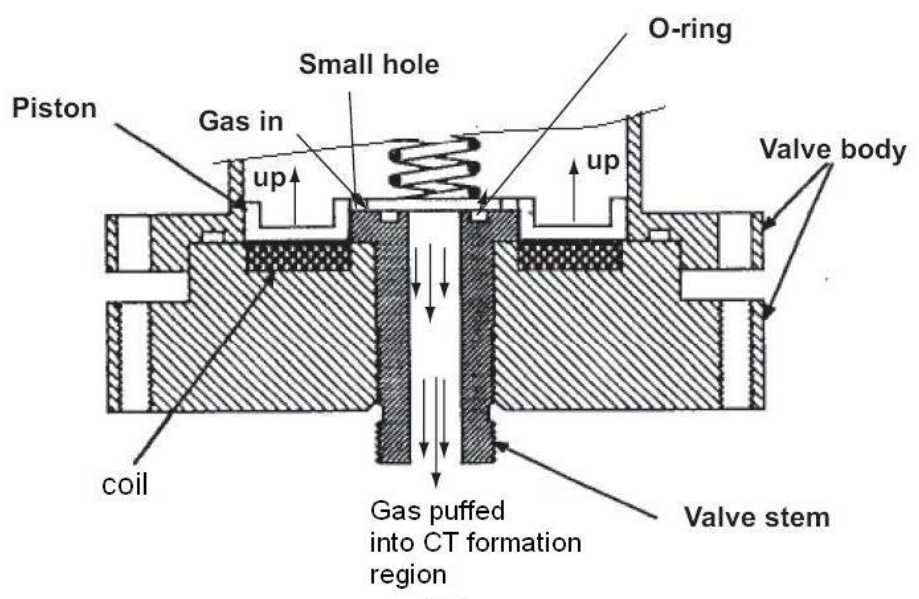

(b)

Figure 3.2: Diagrams of the gas valves showing a) the general valve schematic and b) an expanded schematic of the spring/piston system.

Calibration is performed one gas valve at a time. Three dummy planar coils are 
used in conjunction with the gas valve being calibrated. Calibration ensures that the gas is distributed evenly in the formation region. To facilitate this, each gas valve is connected to the capacitor bank via banana plugs, which can easily be unplugged and moved from the gas valves and the dummy coils.

It was found, throughout the duration of the project, that the valves' calibration did not remain constant. During full, continuous operation, the valves required recalibrating every $\sim 2$ days. The reason for this phenomenon is not known as the deviation for each valve was never consistent. Sometimes an increased gas throughput was observed, and sometimes a decrease was observed.

\subsubsection{Electrical System}

Two identical, $20 \mu \mathrm{F}$, capacitor banks (four, $5 \mu \mathrm{F}$ capacitors connected in parallel) are used for the formation and acceleration discharges in USCTI. Each bank can be charged up to a maximum of $25 \mathrm{kV}$, although they have never been operated at such high voltage. There is a set time delay of several microseconds between the two discharges during operation, to ensure breakdown of the hydrogen gas and formation of a free CT before it is accelerated along the gun. A $1 \mathrm{kV}, 600 \mu \mathrm{F}$ bank is used to fire the four gas valves in parallel, and the solenoid is fired using a $500 \mathrm{~V}, 5000 \mu \mathrm{F}$ bank.

The formation capacitor bank contains the energy used to breakdown the hydrogen gas as well as heat the plasma and form part of the magnetic structure of the CT. The acceleration bank provides the CT with the kinetic energy required to penetrate the tokamak magnetic field, as discussed in Section 2.4.2. Both of these banks must provide a large current, and so are each switched by several 'A' sized ignitron (NL-7703EHV) in parallel and deliver their current via several large RG217/U coaxial cables. Each ignitron is associated with one of the capacitors on the bank. It is arranged in this way to deliver a large current to the injector and to reduce the overall inductance of the transmission line.

The solenoid's magnetic fields must penetrate the stainless steel inner formation electrode. To do this, its capacitor bank must be fired well in advance of the forma- 
tion's to allow sufficient time for its magnetic fields the time to penetrate through the stainless steel electrode. This necessitates its large capacitance $(5 \mathrm{mF})$ for a long discharge duration. The capacitor bank is switched using a Silicon Controlled Rectifier (SCR).

The gas valve capacitor bank was designed to deliver a short current pulse to briefly open the four valves. It is also switched via an SCR.

The formation and acceleration banks are triggered by transformers, whose primary current is provided through the discharge of capacitor banks which are triggered by krytrons, cold-cathode gas filled tubes that use an arc discharge to switch high currents and voltages. Table 3.1 provides a list of the parameters for the USCTI capacitor banks. After upgrading the power supplies, the charge times for all the capacitor banks were decreased.

Table 3.1: Typical capacitor bank settings and charging times before and after modifications.

\begin{tabular}{|c|c|c|c|c|c|}
\hline \multirow{2}{*}{ Capacitor Bank } & $V_{\max }(\mathbf{k V})$ & $V_{\text {set }}(\mathbf{k V})$ & $\mathbf{C}(\mu \mathbf{F})$ & \multicolumn{2}{|c|}{ Charge Time (s) } \\
\cline { 4 - 6 } & & & & Initial & Modified \\
\hline Formation & 25 & 18 & 20 & 120 & 0.7 \\
Acceleration & 25 & 15 & 20 & 120 & 0.7 \\
Solenoid & 1 & 0.34 & 5000 & 180 & 0.6 \\
Gas Valves & 1.5 & 0.875 & 600 & 180 & 0.85 \\
Krytron & 8 & 4 & 1 & 10 & 1 \\
\hline
\end{tabular}

\subsection{Modifications to USCTI}

Many modifications to USCTI were performed in order to make repetitive operation a reality. Some of these changes were planned for, such as getting high-current charging power supplies, others were not foreseen, and were changed throughout the project, such as the grounding scheme. 


\subsubsection{Main Power Supplies}

The first stepping stone for repetitive operations was to overcome the long charging time of the capacitor banks. This was achieved by replacing the existing power supplies on USCTI. For the formation and acceleration banks, Lambda 802 power supplies were purchased, which have a maximum charging voltage of $25 \mathrm{kV}$ and a peak power rating of $8000 \mathrm{~W}$ each. The solenoid and gas valve supplies were replaced with Lambda 402 units, which have a peak power rating of $500 \mathrm{~W}$ each. The solenoid supply has a maximum charging voltage of $500 \mathrm{~V}$ and the gas valve supply $1000 \mathrm{~V}$.

Charging times for the power supplies are calculated from [42]

$$
T_{\text {charge }}=\frac{0.5 C_{\text {load }} V_{\text {charge }} V_{\text {rated }}}{P_{\text {peak }}},
$$

where $T_{\text {charge }}$ is the charge time in seconds, $P_{\text {peak }}$ is the peak power rating in watts, $C_{\text {load }}$ is the capacitance in Farads, $V_{\text {charge }}$ is the voltage in volts and $V_{\text {rated }}$ is the power supply rating in volts. From Equation 3.1, therefore, the new supplies have the charging times shown below.

The Lambda 802 supplies, for the formation and acceleration banks, have a charging time of

$$
T_{\text {charge }}=0.5 \times 20 \mu \mathrm{F} \times \frac{25000 \mathrm{~V} \times 25000 \mathrm{~V}}{9000 \mathrm{~W}}=0.7 \mathrm{~s},
$$

which is a worst case scenario, as for operation, charging voltage is less than $18 \mathrm{kV}$. The Lambda 402 supplies have a charging time of

$$
T_{\text {charge }}=0.5 \times 600 \mu \mathrm{F} \times \frac{1000 \mathrm{~V} \times 1000 \mathrm{~V}}{500 \mathrm{~W}}=0.6 \mathrm{~s}
$$

for the gas valve bank and

$$
T_{\text {charge }}=0.5 \times 5000 \mu \mathrm{F} \times \frac{340 \mathrm{~V} \times 500 \mathrm{~V}}{500 \mathrm{~W}}=0.85 \mathrm{~s}
$$

for the solenoid bank.

Furthermore, because the solenoid is positioned close to the inner formation electrode within USCTI, in order to mitigate arcing between the two, the entire solenoid 
system was made to electrically float. The solenoid's operating voltage is $340 \mathrm{~V}$ and the formation bank sits at upwards of $18 \mathrm{kV}$. With the solenoid floating, when the entire system is charged, the solenoid would sit at a voltage relative to the formation electrode, which would not have sufficient potential difference to cause an arc.

The original electrical system design implemented an RC filter to decouple the formation and acceleration power supplies from the discharging circuits (where $\mathrm{R}$ $=100 \Omega$ and $\mathrm{C}=250 \mathrm{nF}$ ). Since a $100 \Omega$ resistor would limit the charging current, to protect the Lambda 802 power supplies, it was replaced with a $5.5 \mathrm{mH}$ inductor, shown in Figure 3.3. Combined with the $250 \mathrm{nF}$ capacitor, the LC circuit blocks any high frequency ringing current from damaging the supplies, but allows the comparatively low frequency charging current to pass unheeded.

The cut-off frequency for the new LC filter is $f_{0}=27 \mathrm{kHz}$, calculated from

$$
f_{0}=\frac{1}{2 \pi \sqrt{L C}} .
$$

The frequency of the CT discharging system is on the order of $\sim 60 \mathrm{kHz}$ for the acceleration bank and $\sim 90 \mathrm{kHz}$ for the formation bank, which is found from their respective current waveforms, shown in Figure 3.4. This filter, then, allows for the charging current, which is on the order of $1 \mathrm{~Hz}$, to pass unheeded to the capacitor banks, and prevents the ringing effect coming from the discharging banks from damaging the power supplies.

\subsubsection{Grounding and Relays}

It was initially intended that the new Lamdba 801 power supplies be grounded through the pre-existing grounding scheme. Upon installing them, it was discovered that safety regulations at the university require that their ground be connected directly through their power cord during operation. It was found through trial that the power supply ground (through the power cord) is connected at an unknown location to the lab ground that was being used for the other USCTI components. The grounding for all USCTI equipment, therefore, was re-routed to the Lambda 


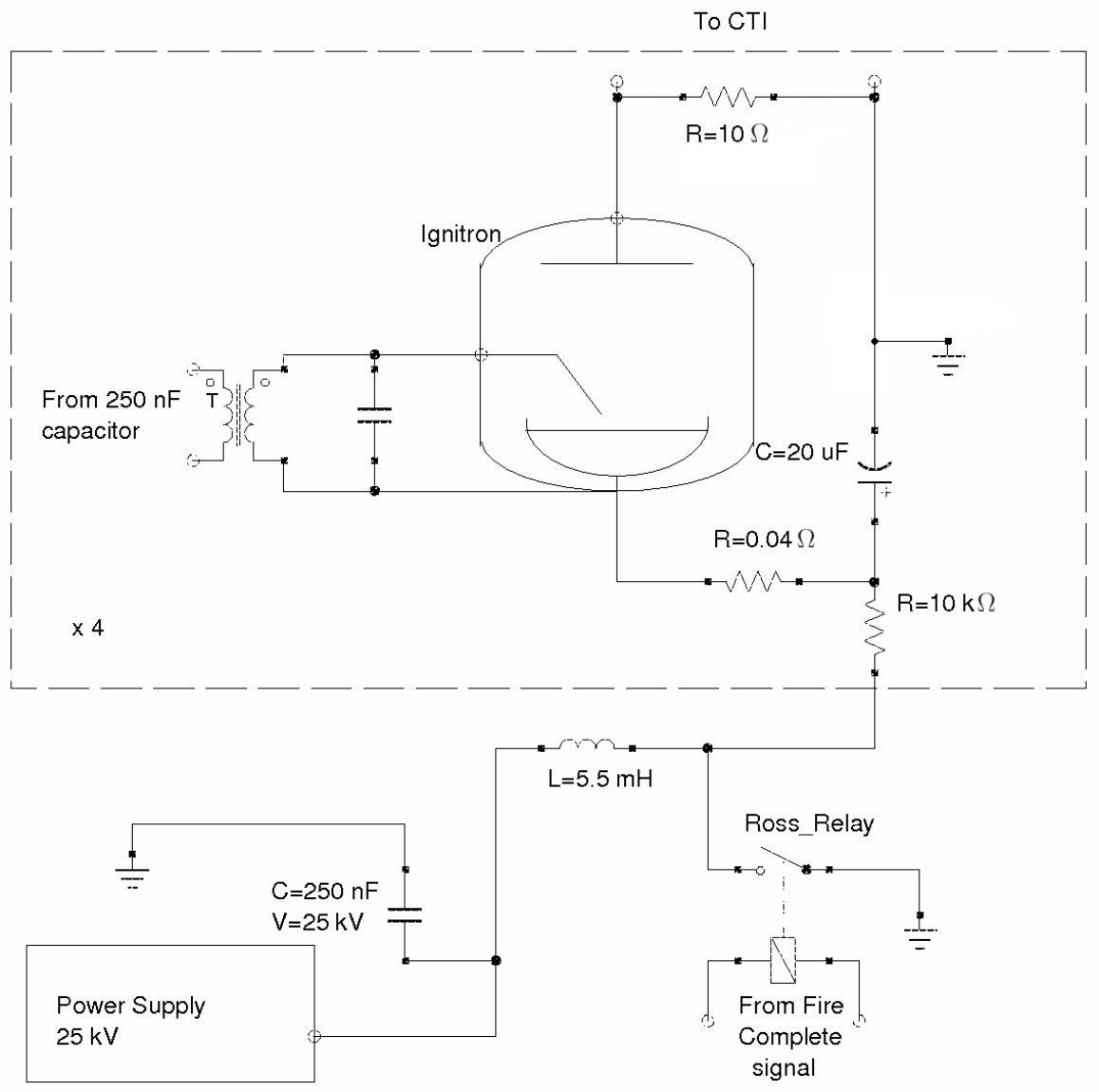

Figure 3.3: Schematic of the charging and discharging system for the formation and acceleration capacitor banks. 

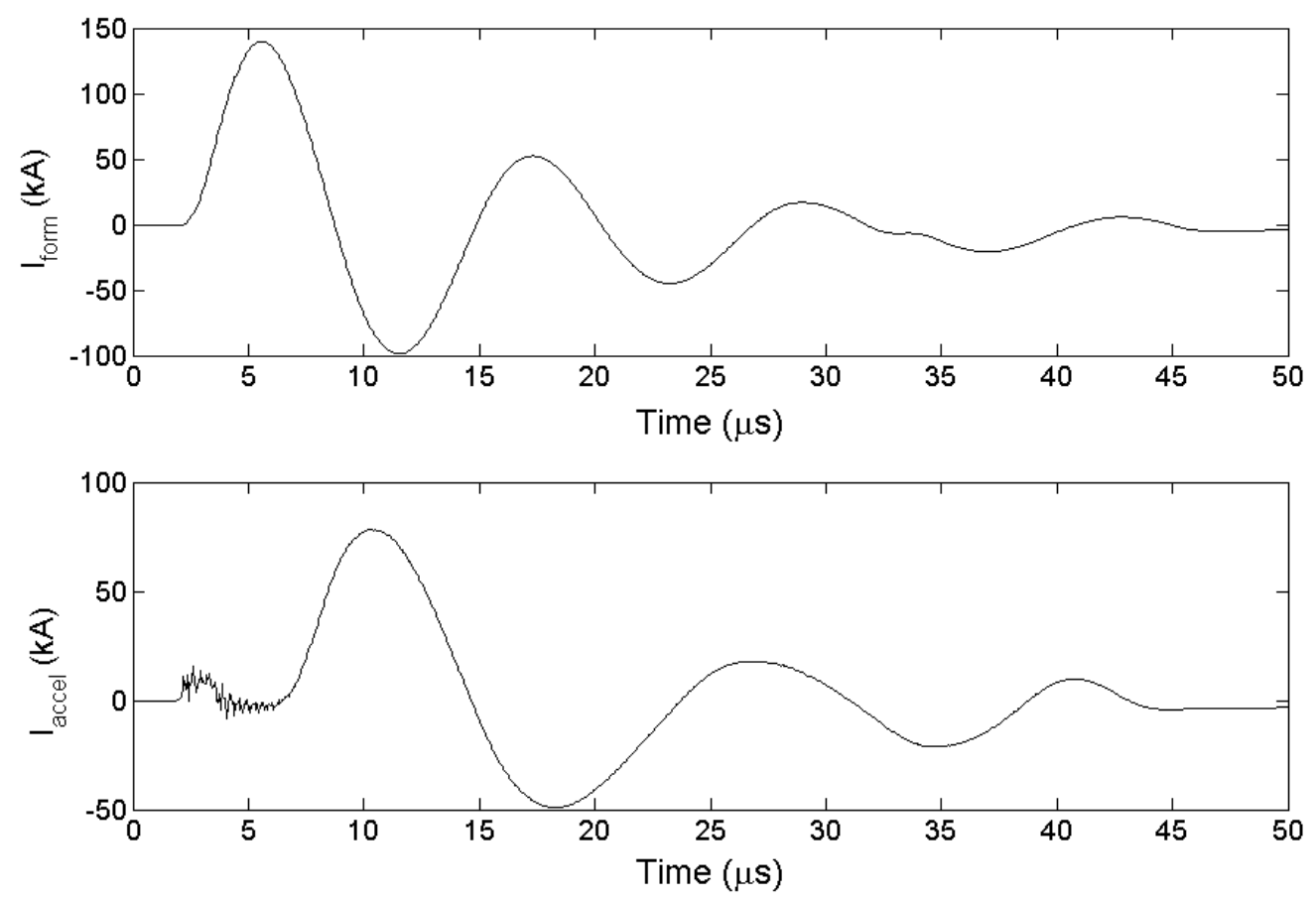

Figure 3.4: Diagram of the current waveforms for the formation and acceleration discharges. 
802 supplies' ground to avoid a ground loop.

Modifications were also made to the capacitor banks grounding. Ross relays were used to lift the grounding of the capacitor banks during discharges in previous experiments. A set of Ross relays that ordinarily switched open during firing to float the charged solenoid and gas valve capacitor banks were removed. Because repetitive firing required fast switching, they were seen as redundant. As well, override switches were installed in order to switch the formation and acceleration capacitor banks from single shot to repetitive firing. This essentially stopped a grounding relay from opening each time USCTI was fired, facilitating fast firing.

It was noticed that arcing had developed across the Ross relays on the formation and acceleration capacitor banks when the relays were switched open to disconnect the banks from ground. The arcing was occurring on the grounding relays because they were switched out of synch with the the power supplies. This was not observed in the original configuration, as the original supplies limited the charge to $\leq 10 \mathrm{~mA}$, which was not high enough to cause such an arc. To mitigate this, a $33 \mu \mathrm{F}$ capacitor and a $100 \Omega$ resistor were placed on the inhibit signal of the power supplies as well as a $470 \mu \mathrm{F}$ capacitor and $100 \Omega$ resistor on the control signal for the grounding relays. These are shown in Figure 3.5.

When the charging signal is issued, the Ross relay power is activated, causing the relay to open, simultaneously, the inhibit signal is deactivated, allowing the power supply to begin charging. The charged $33 \mu \mathrm{F}$ capacitor on the inhibit circuit discharges through the $(0.1 \mathrm{k} \Omega+1.2 \mathrm{k} \Omega)$ resistor, with a time constant $\tau=43$ $\mathrm{ms}$, forcing the power supplies to remain inhibited for slightly longer $(\sim 10 \mathrm{~ms}$, as measured) than the time taken for the Ross relays to open. When the fire complete signal is issued the inhibit signal is reactivated, inhibiting the power supplies from charging. The Ross relay power is also deactivated, causing the relays to ground the capacitor banks. The charged $470 \mu \mathrm{F}$ capacitor discharges through the $50 \Omega$ resistor, with a time constant of $\tau=23 \mathrm{~ms}$, causing the Ross relays' power to keep them open slightly longer $(\sim 100 \mathrm{~ms}$, as measured $)$ than it takes for the power supplies to become inhibited after firing. 


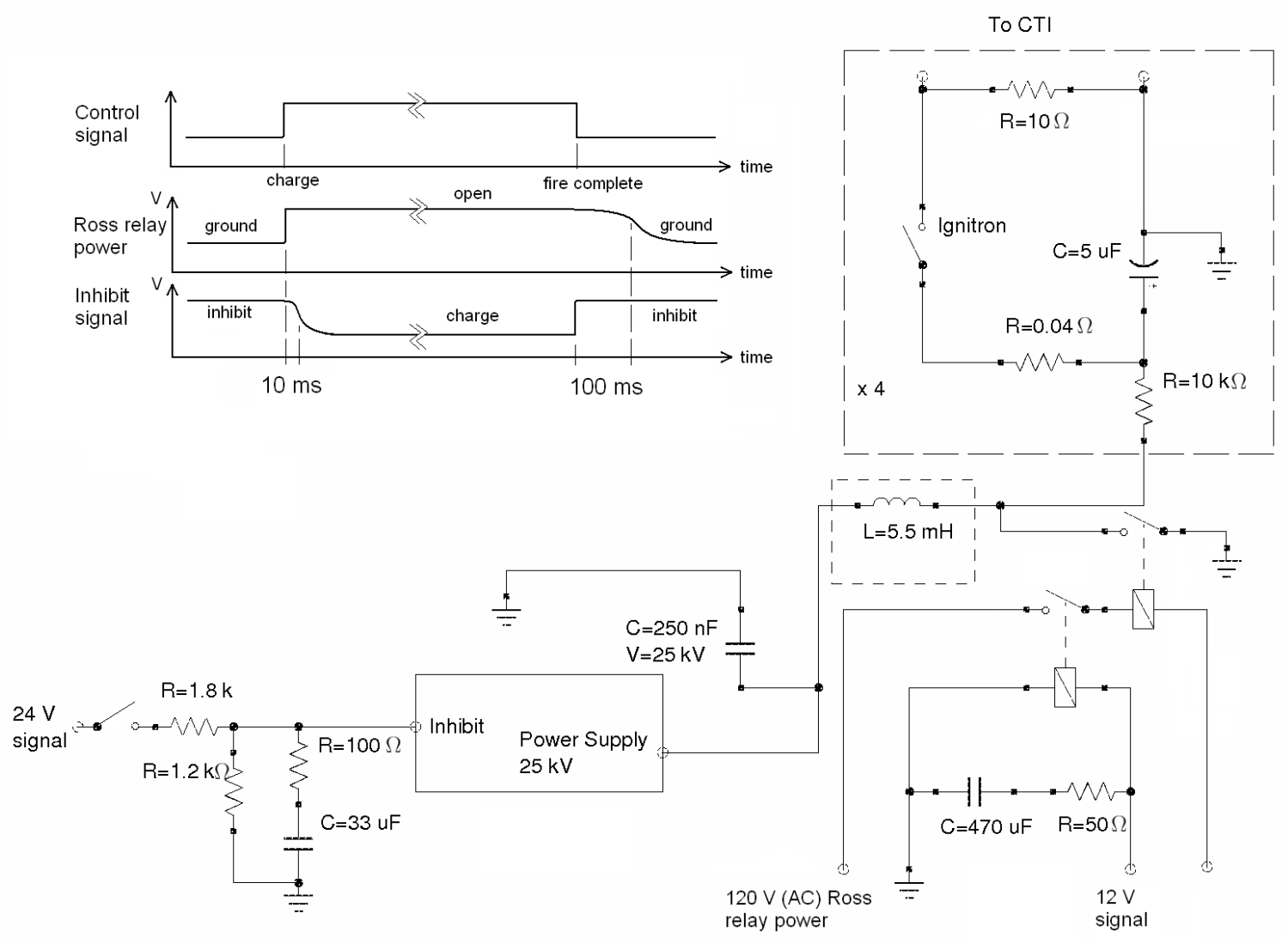

Figure 3.5: Schematic of the grounding relays on the formation and acceleration capacitor banks (both banks are similar). The capacitors which enable prolongation of the power supply inhibit signal as well as open ground are shown. The figure in the upper left corner shows the scheme for the Ross relay and inhibit signal overlapping. 


\subsubsection{Krytrons}

The formation and acceleration banks are activated through ignitrons, so that a fast discharge is achieved. The ignitrons themselves are switched via two krytrons, one for the formation and one for the acceleration supply. The krytrons are each fired using a $1 \mu F, 8 \mathrm{kV}$ capacitor bank. As shown in Table 3.1, the krytron capacitor banks' charging time of about $10 \mathrm{~s}$ was not sufficient for the scope of this project. The original intention was to use the original, Glassman, power supplies from the formation and acceleration banks (that were no longer being used) to charge the krytron's capacitors. Upon testing, however, they were found to present pre-triggering issues.

When the Glassman power supplies were turned on to charge the krytron capacitor banks, they would charge up to a point (approximately full voltage) and then the krytrons would immediately fire, without being triggered. The cause of this phenomenon was not able to be determined, although it is believed to be a problem with the fact that the Glassman power supplies are operated in a constant current mode, and that this somehow affected the krytrons' circuitry.

To circumvent this hurdle, two small EMCO F50 power supplies were purchased to replace the existing krytron supplies. The new supplies have an output current of $2.8 \mathrm{~mA}$, three times higher than the original supplies. In addition, to provide faster charging, a $5 \mathrm{M} \Omega$ charging resistor was replaced with a $500 \mathrm{k} \Omega$ resistor, shown in Figure 3.6.

The krytrons themselves are an issue which must be contended with. The specific devices used on USCTI have been in use for some time and have an operational lifetime of $\simeq 50000$ firings. Before the commencement of this project, the krytron on the formation bank had already been replaced. Evidence of the disparity between formation and acceleration krytrons was rife throughout the experiment. The acceleration bank's krytron had both a longer charging time as well as a lower reliability (when operated at an equal voltage to the formation bank's krytron). To counter the reliability issues, the acceleration bank's krytron was set to charge at a slightly 


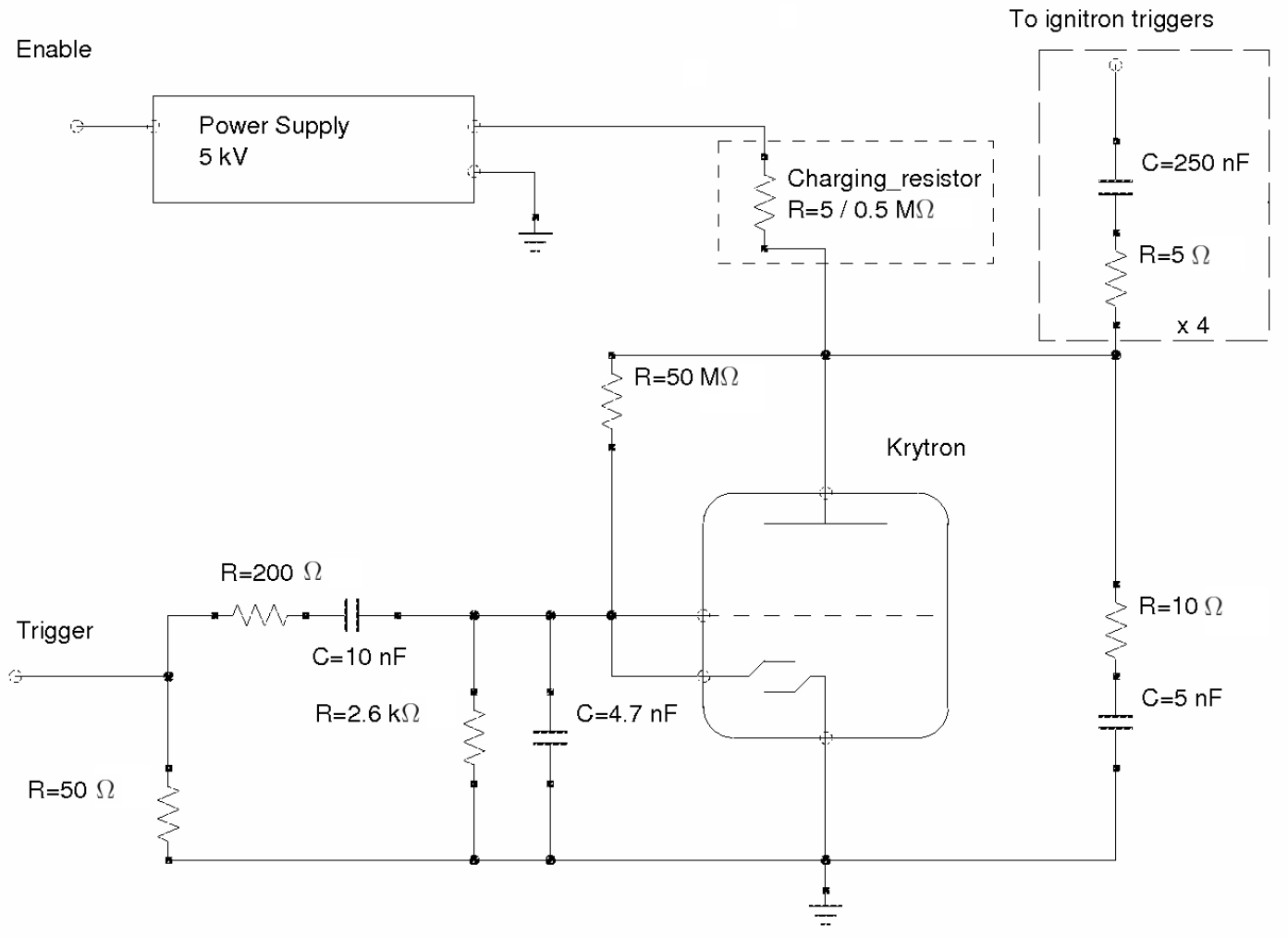

Figure 3.6: The krytron charging and discharging system. The charging resistor, which was changed to allow faster charging, is shown surrounded by a dotted line. 
higher voltage than that of the formation bank $(4.2 \mathrm{kV}$ as opposed to $4 \mathrm{kV})$.

In the past, when USCTI was only fired once every three minutes, this might not have been an issue. In a repetitive mode, it is easy to see that their lifetime is running short. On top of this, krytrons are not available on the market and they would therefore need to be replaced in the future.

\subsubsection{Gas Valves}

During repetitive operation experiments on USCTI, one of the major setbacks was related to the gas valves. After an inconsistent number of firings (between 50 to 200) the gas valves would arc, leaking hydrogen to atmosphere. Through analysis, it was found that the arc was being created across the gas valve's coil and the gas valve's body. When the arc occurred, the process would not only cause the arc but would puncture a hole in the diaphragm.

The diaphragm acts as a wall in the gas valve. It holds the planar coil in place and seals hydrogen in the valve. When the diaphragm is punctured, it creates a leak to atmosphere. It is only fortunate that such a puncture does not affect the vacuum chamber.

The most likely cause of this arc is due to the configuration of the gas valves' charging supply. Previously, the gas valves were made to electrically float during the discharge. This means that the voltage on the gas valve coil would range from $\sim 400$ $\mathrm{V}$ on the capacitor bank's positive terminal and $-400 \mathrm{~V}$ on the negative terminal. As explained in Section 3.2.2, the floating relay was removed to facilitate fast firing of the injector. Because of this, the gas valve solenoids would have a voltage of $0 \mathrm{~V}$ on the grounded side, and $+815 \mathrm{~V}$ on the other, from the capacitor bank's positive terminal. This increased voltage between the grounded gas valve body and the elevated coil was too much for the insulation to handle.

When a gas valve would break, more insulation was placed around its (newly replaced) coil. High voltage grease, heat shrink and kapton tape were used on the feedthrough section of the gas valves. The feedthrough was found to be the area where arcing would occur as the coil wire was needed to be bent at this location. 
This would create a weak point for the arc to occur.

Originally, the gas valve coils were made with 22 turns of magnet wire. In order to provide further insulation gaps, all new solenoids are made with only 21 turns. This created a greater air gap along the circumference of the solenoid, and facilitated the addition of further insulation materials to prevent arcing.

\subsection{Summary}

This chapter discussed the USCTI system. The various components of USCTI, it's formation, compression and acceleration sections, as well as its electrical and gas puffing systems, were described. A description of the modifications made to facilitate repetitive operation as well as problems encountered during repetitive CT operation and the steps taken for mitigation was presented. 


\section{CHAPTER 4}

\section{Diagnostics And Data Analysis}

The scope of this project explored the feasibility of firing USCTI in a repetitive mode. In order to determine that a repetitive mode had been properly achieved, diagnostics were needed. These were used in order to test the quality of the CT as it was accelerated along the injector. If the CT had not been of good quality, it could not be properly injected into a tokamak plasma.

The primary needs of diagnostics, then, were to analyze the properties of the CT as it was formed and accelerated. It was necessary to show that the CTs made were in fact spheromak-like. If the proper magnetic fields were not being created, or were degrading over time, the CTs would not be effective at fueling a tokamak plasma. For this purpose, magnetic probes were used to measure the $B_{z}$ poloidal field. For integration with tokamak plasmas, it is the poloidal field which is characteristic of CT formation.

In order for an accelerated CT to penetrate through the tokamak's toroidal magnetic field, as described in Section 2.4, it must have a sufficient velocity. The CTs' time-of-flight across the probes was used to infer that they obtained a sufficient velocity $(\sim 200 \mathrm{~km} / \mathrm{s})$.

Density measurements of the $\mathrm{CT}$ was also needed. If the formation was not consistent, it would cause a CT to be created without the proper density to provide adequate fueling upon integration with a tokamak plasma or without the ability to penetrate into the tokamak core region. By measuring the density of the CT, it could be determined whether or not, over time, the injector's ability to form a proper CT was degrading. Two possible methods for density measurement, which are described in this chapter are Langmuir probes and interferometry. Due to the nature of the 
attachment of USCTI to STOR-M, Langmuir probes were used.

A new signal digitizer was purchased in order to facilitate multiple channel acquisition and a data acquisition program was created that was able to store data fast enough as USCTI was fired. Data was then analyzed using software developed using MATLAB. Data was examined to determine quality of the CT plasma as well as reproducibility; each acquired signal was correlated with its mean in order to observe trends in multi-shot tests.

This chapter begins by discussing the diagnostics of CT injectors and those used in the experiment, as well as a description of their corresponding theory. An explanation of the acquisition methods used is also presented. Finally, a description of the postprocessing methods used for analysis is presented.

\subsection{Diagnostic Theory}

Magnetic probes are crucial to determine the CT formation. This enabled the determination of velocity and length of the CT during each firing. Data can then be analyzed to determine reproducibility. Time-of-flight analysis of the CT magnetic field waveform recorded by magnetic probes positioned along the acceleration region was used to infer velocity. Density was measured using a triple Langmuir probe. Figure 4.1 [26] shows a schematic of the probe positions on the CT injector.

The Figure shows a downward view of the injector. The magnetic probes, at positions $\mathrm{z}=0,22,43$ and $65 \mathrm{~cm}$ are located along the top of the injector. The triple Langmuir probe, located at $\mathrm{z}=85 \mathrm{~cm}$, is positioned $105^{\circ}$ azimuthally from the top of the injector. Also shown is the location of the bellows, which are necessary in order to attach USCTI to STOR-M. The ceramic break is used to insulate the tokamak ground and the USCTI ground, preventing any ground loops which may induce electromagnetic noise during tokamak and/or CT discharges. 


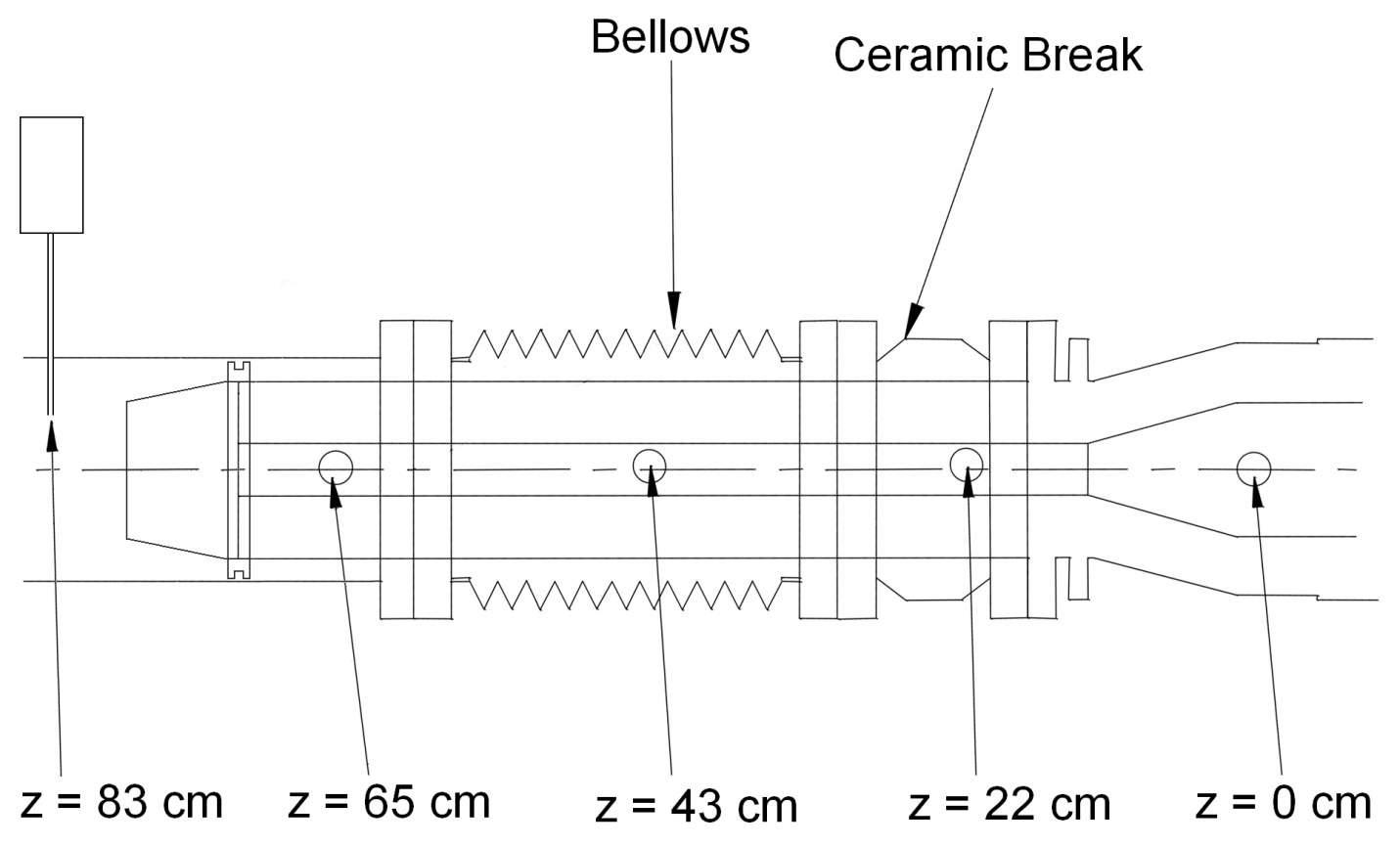

Figure 4.1: A schematic of the USCTI acceleration region, showing the locations of the probes used. 


\subsubsection{Magnetic and Current Probes}

Pick up coils are used as magnetic probes, converting a magnetic signal from the CT into a voltage output through Faraday's Law of induction based on the formula $V=-N d \phi / d t$, where $\phi=\int \vec{B} \cdot d \vec{s}$ is the magnetic field flux enclosed by the coil, and $N$ is the number of windings in the coil. At each probe location, two coils (6 turns of AWG32 wire of $8 \mathrm{~mm} \times 3 \mathrm{~mm}$ cross section) are placed perpendicularly to measure $B_{z}$ and $B_{\phi}$.

The magnetic field is found from the probes by using

$$
V=N A \dot{B}
$$

where $V$ is the measured voltage signal, $N$ is the number of turns, $A$ is the cross sectional area of the loop and $\dot{B}$ is the time variation of the magnetic field, $B$, given by $B=B_{0} e^{-t / R C}$. Because passive $\mathrm{RC}$ integrators were used, the equation can be rewritten as

$$
V_{\text {int }}=\frac{-N A B_{0}}{R C}
$$

where $\mathrm{RC}$ is the time constant of the integrator [43] and is chosen to be much larger than the time scale of observation. Figure 4.2 shows a schematic of the RC integrators used to acquire the magnetic probe signals on USCTI.

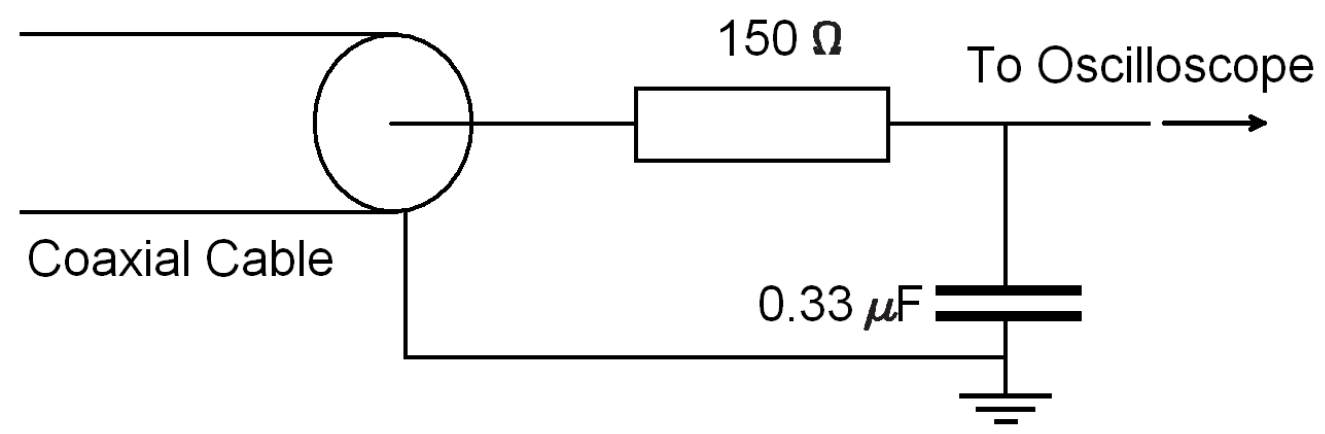

Figure 4.2: Schematic of the integrators used on USCTI.

Probes are positioned along the injector on the inner surface of the outer elec- 
trode, one at the formation region $(z=0 \mathrm{~cm})$, and three along the acceleration region $(z=22,43$ and $65 \mathrm{~cm})$. An average velocity was calculated using basic kinematics by dividing the distance between two of the three acceleration probe positions $\left(d_{22}\right.$, $d_{43}$ and $\left.d_{65}\right)$ by their corresponding time of flight $\left(t_{22}, t_{43}\right.$ and $\left.t_{65}\right)$. Normally, the last two probes are used as

$$
v_{\text {avg }}=\frac{d_{65}-d_{43}}{t_{65}-t_{43}},
$$

yielding the final muzzle velocity.

Rogowski coils are used to measure the discharging current of the capacitor banks. A Rogowski probe takes advantage of the magnetic field generated by a current. It is built by using a toroidal magnetic probe which can be wrapped around a wire (or other current carrier). The flux passing through the center of the solenoid can be described as

$$
\Phi=n \oint_{l} \int_{A} d A \mathbf{B} \cdot \mathbf{d} \mathbf{l}
$$

where $n$ is the number or turns of wire per unit length in the coil, $d A$ is an infinitesimal portion of the cross sectional area, $A$, of the coil, $I$ is the current encircled by toroid, $\mathbf{d l}$ is an infinitesimal portion of the length, $l$ of the solenoid, $\mu$ is the magnetic permeability of the medium in the solenoid and $\mathbf{B}$ is the magnetic field.

From Ampères law

$$
\oint_{l} \mathbf{B} \cdot \mathbf{d l}=\mu I
$$

and so the flux reduces to

$$
\Phi=n A \mu I
$$

and the voltage measured by the Rogowski coil is then given by [43]

$$
V=\dot{\Phi}=n A \mu \dot{I}
$$

Figure 4.3, from Ramboz [44], provides a diagram of the basics of a Rogowski coil.

Because the frequency of the current is so high, $(\sim 100 \mathrm{kHz}$, see Section 3.2.1), self-integrators are used on the Rogowski coils, and the equation can then be rewrit- 


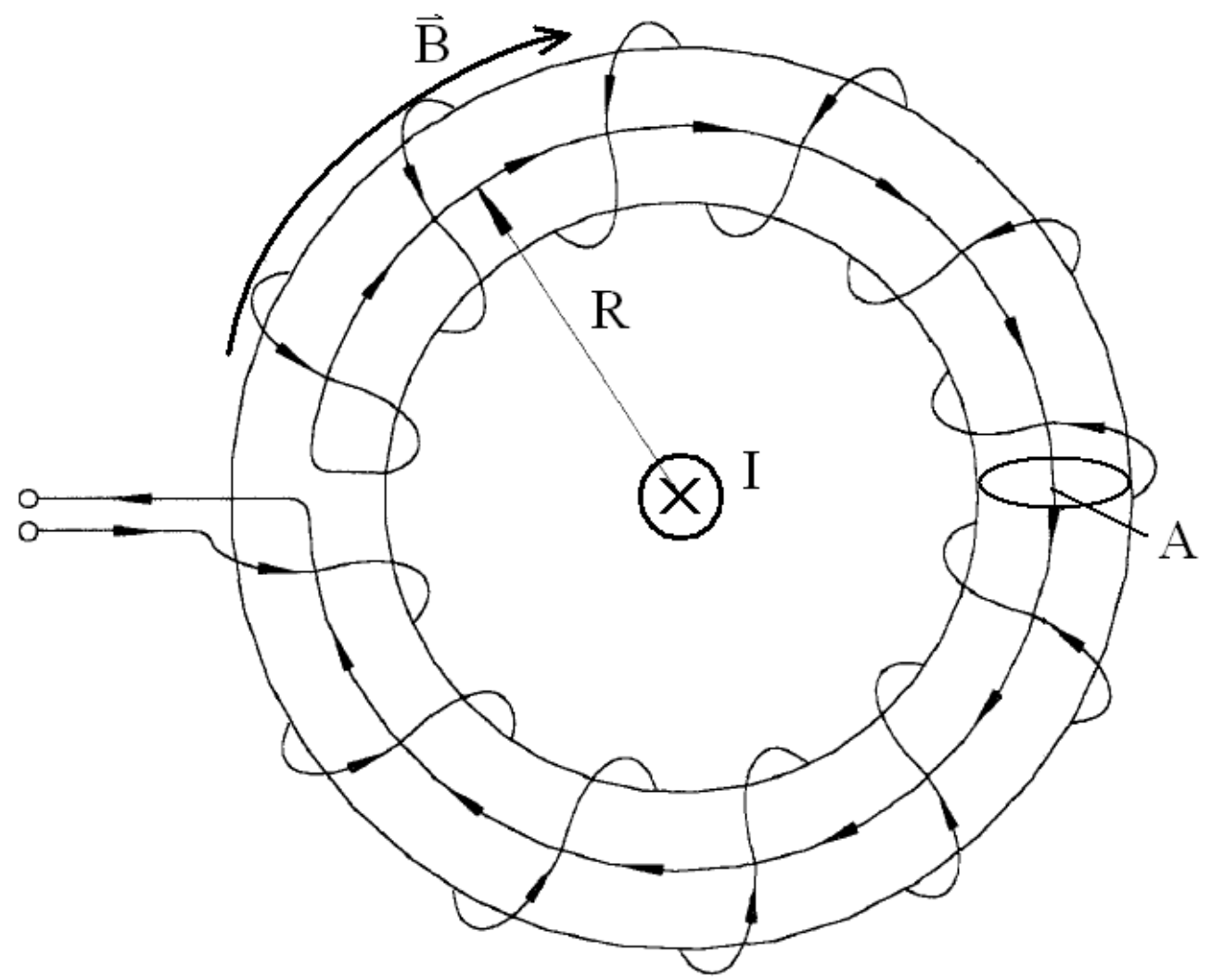

Figure 4.3: Diagram of a rogowski coil showing the current $I$, going into the page and causing the magnetic field $\mathbf{B}$ at the Rogowski coil of radius $R$ and cross sectional area, $A$. 
ten as

$$
V=\frac{N \mu_{0} \pi a^{2}}{2 \pi R L} R_{0}
$$

where $R$ and $a$ are the major and minor radii of the coil, $L$ is its inductance, and $R_{0}$ is the resistance of the resistor used. Figure 4.4 shows a schematic of the type of self-integrators used to acquire the Rogowski coil signals.

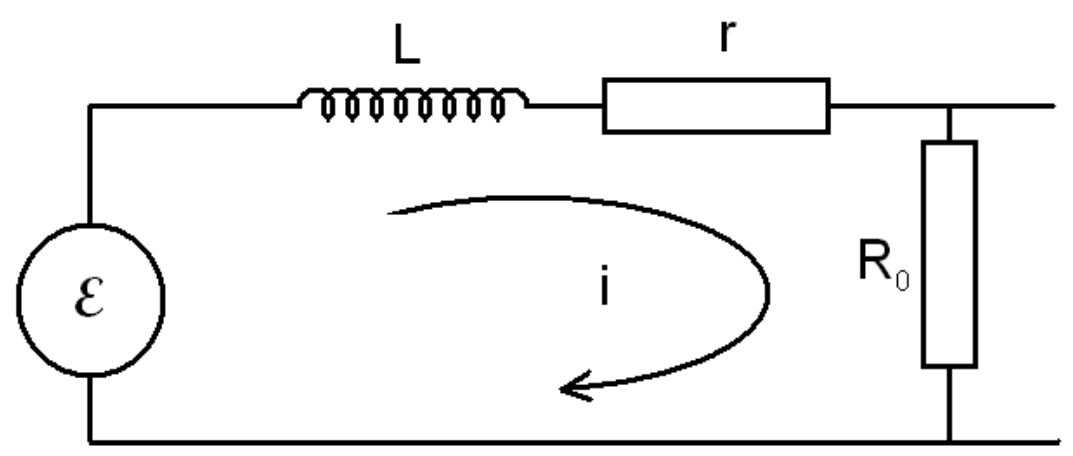

Figure 4.4: Schematic of a self-integrator used on the Rogowski coils. The resistor $r$ is the internal resistance of the probe.

An added benefit of using a Rogowski coil to measure a current is that no physical linkages must be made to interrupt the current path the system. Unwanted grounding of a system can therefore be avoided.

\subsubsection{Density Measurements}

A Langmuir probe is simply a conductor biased with respect to the chamber wall or another nearby conducting probe and inserted into the plasma. It can be used to measure plasma temperature, density and other parameters.

By ignoring the magnetic fields in the plasma, as well as assuming a Maxwellian distribution for the ions and electrons, the current density flowing into the probe is

$$
J=-J_{i 0}+J_{e 0}(1-\Gamma) e^{\frac{e\left(V-V_{s}\right)}{T_{e}}}
$$


where $J_{i}$ is the ion current density, $J_{e 0}$ is the electron saturation current density, $\Gamma$ is the secondary emission rate of the probe conductor (typically, tungsten is chosen, making $\Gamma$ negligible) $T_{e}$ the electron temperature (in $\mathrm{eV}$ ), $V$ is the probe potential, $V_{s}$ is the plasma space potential and $V<V_{s}$ before entering the electron saturation current region. An ideal I-V curve for a Langmuir probe is presented in Figure 4.5.

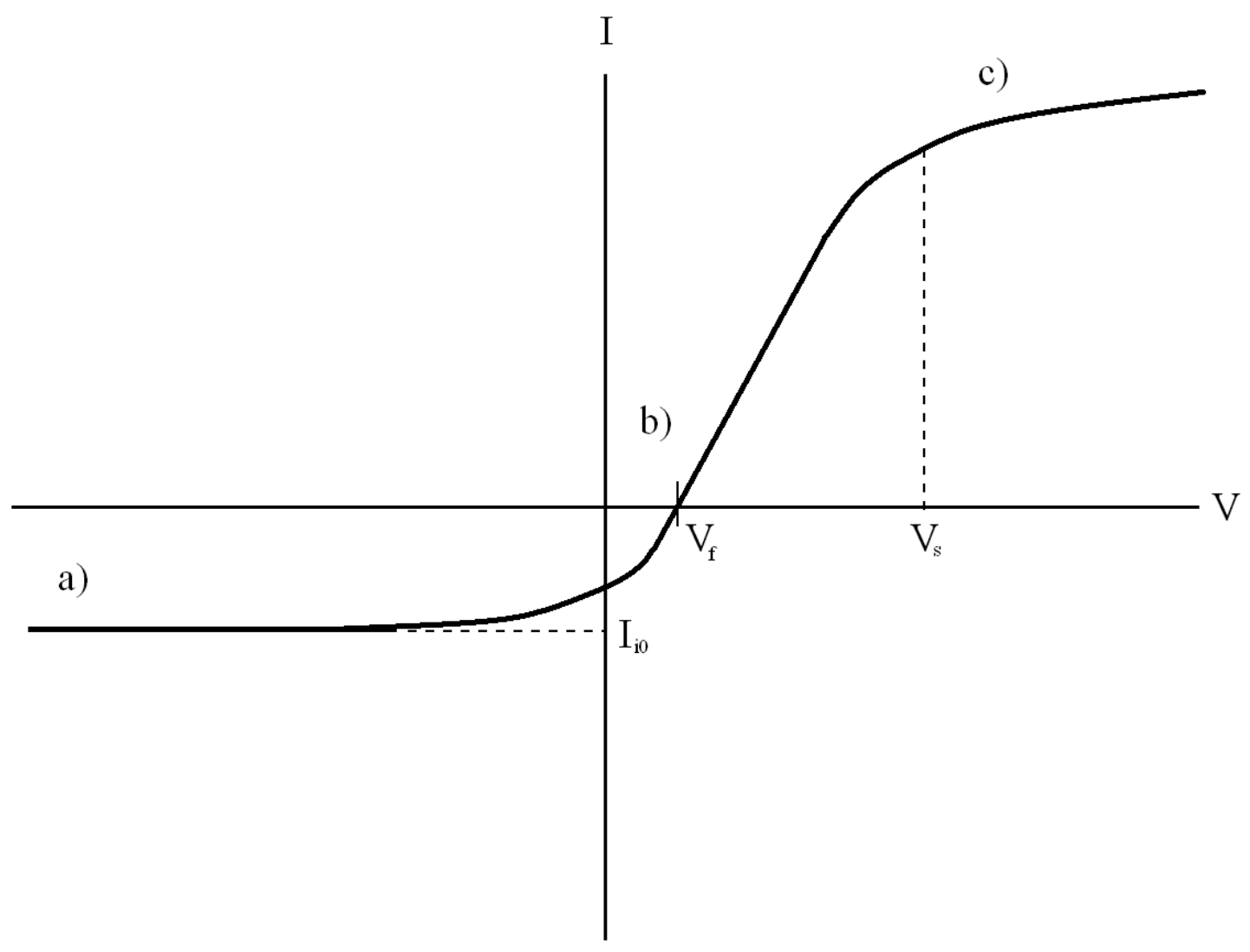

Figure 4.5: Typical I-V curve showing a) ion saturation current region, b) floating potential $V_{f}$ and transition region and c) electron saturation current region.

If $V-V_{s} \gg k T_{e}$, then the probe current will be limited by the electron saturation current, given by

$$
I_{e 0}=\frac{1}{4} e n_{e} A \sqrt{\frac{8 T_{e}}{\pi m_{e}}},
$$

with $n_{e}$, the electron density, $A$, the probe collection area and $m_{e}$ the electron mass. At this point, due to the probe's positively biased voltage, it will repulse ions and 
collect the available electrons entering the plasma sheath, and so the collected current becomes unaffected by changes in the probe's voltage (hence the plateau effect seen in Figure 4.5).

The floating potential, $V_{f}$ of a probe is, relatively, self explanatory. It is the potential at which the total electron current collected is equal to the ion current, and therefore the total current collected is zero. It is the potential that a probe inserted into a plasma would acquire if the probe was electrically isolated (i.e. floating). This is a consequence of electrons having higher mobility than ions. They will be first to be collected by the probe, quickly giving it a negative voltage with respect to the plasma potential, $V_{s}$. Subsequently, it will attract ions and repulse electrons.

When the potential of the probe is brought below the floating potential, $\left(V<V_{f}\right)$, then electrons will be repelled from the probe's surface. A net negative current will result. When the probe is biased sufficiently negative, then no electrons will be collected. This, naturally, is called the ion saturation current, $I_{\text {sat }}$. It is also referred to as the Bohm current, and is visually represented by the plateau on the left side of Figure 4.5.

Given a probe with collection area, $A$, and where ions entering the sheath region have a drift velocity $u_{0} \geq\left(k T_{e} / m_{i}\right)^{1 / 2}$, the ion current collected by a negatively biased probe is

$$
I_{i 0}=n_{s} e A\left(\frac{k T_{e}}{m_{i}}\right)^{1 / 2}
$$

where $n_{s}$ is the density of the plasma at the edge of the sheath, $m_{i}$ is the ion mass and $T_{e}$ the electron temperature. If $u_{0}=\left(k T_{e} / m_{i}\right)^{1 / 2}$ exactly at the sheath edge, then there must be a presheath potential of $|\phi| \geq \frac{1}{2}\left(k T_{e} / e\right)$. The potential at the edge is then $\phi_{s} \simeq-k T_{e} / e$ relative to the plasma. Therefore

$$
n_{s}=n_{0} e^{\frac{e \phi}{k_{e}}}=n_{0} e^{-1 / 2}
$$

where $n_{0}$ is the unperturbed plasma density at infinity. The measured ion saturation current, or Bohm current, is then given by [40]

$$
I_{\text {sat }}=n_{0} \exp (-1 / 2) e A\left(\frac{k T_{e}}{m_{i}}\right)^{1 / 2} .
$$


Knowing electron temperature, which can also be determined using a Langmuir probe, density can then easily be calculated.

\subsubsection{Assumptions to Langmuir Probe Theory}

The Langmuir probe current derivations assume that the plasma in question is collisionless and unmagnetized. When collisions between particles occur, the probe's collected current will be affected. Specifically, if collisions occur on a scale smaller than the plasma's sheath length, on the order of the Debye length, particles could be accelerated toward the probe, but, due to collisions, be scattered away before they are actually collected by the probe. As well, when a plasma is magnetized, particles will form orbits in the magnetic field, further increasing the possibility of altering a probe's measurements.

In order for a plasma to be considered collisionless, it must be shown that the mean free path, $l$, is much larger than the plasma's Debye length, $\lambda_{D}$. A plasma's Debye length is a measure of the distance over which a charge carrier can screen out electric fields. The Debye length is given by

$$
\lambda_{D}=\sqrt{\frac{\epsilon T_{e}}{e^{2} n}}
$$

and the mean free path can be estimated by taking the ratio of the electron thermal speed,

$$
v_{T e}=\sqrt{\frac{2 e T_{e V}}{m_{e}}}
$$

and the electron-ion collision frequency

$$
\nu_{e i} \simeq 2 \times 10^{-6} \frac{Z n_{e} \ln \Lambda}{T_{e V}^{3 / 2}} .
$$

as simply

$$
l \simeq \frac{v_{T e}}{\nu_{e i}} .
$$

On top of this, the poloidal and toroidal magnetic fields present in the plasma create orbits in the electron and ion motions. In order for the theory to apply, the 
probe used must have a size such that the rotating plasma particles can be treated as having a relative linear motion when they strike the probe tip.

The particle's orbit inside a magnetic field is described using the Larmor radius,

$$
r_{l}=\frac{m v_{\perp}}{q B}
$$

where $m$ is the mass of the particle (in a hydrogen plasma case, a proton or electron), $v_{\perp}$ is its velocity perpendicular to $B$, the magnetic field and $q$ is the particle's charge. For the theory to hold true, the probe area, or probe's diameter $a$, in the case of a cylindrical probe such as is used on USCTI, must be chosen such that $r_{l} \gg a$.

For USCTI, with a magnetic field of $\sim 0.2 T$, density $n_{e} \sim 10^{20} \mathrm{~m}^{-3}$ and electron temperature $T_{e} \sim 10 \mathrm{eV}$ as shown in chapter 5 it can be found that $\lambda_{D} \simeq 2.5 \mu \mathrm{m}$ and $l \simeq 2 \mathrm{~cm}$, making the plasma essentially collisionless. The ion larmor radius $r_{i} \simeq 2 \mathrm{~mm}$ and the electron larmor radius $r_{e} \simeq 0.1 \mathrm{~mm}$. The probe used on USCTI has a diameter of $0.5 \mathrm{~mm}$, and so, because the probe is measuring the ion saturation current (eq 4.13), the plasma can be said to have relative linear motion when striking the probe.

\subsubsection{Triple Langmuir Probe}

When measuring long duration plasmas, a single Langmuir probe will normally suffice. The probe's bias voltage can be swept across a range of voltages (typically $-100 \mathrm{~V} \rightarrow+100 \mathrm{~V})$. In this way, $T_{e}$ can be inferred from the slope of the resulting $I-V$ curve $[40,45,46]$. On the STOR-M tokamak, for example, plasma duration is $\sim 30 \mathrm{~ms}$. This is more than enough time for a Langmuir probe to be swept across a varying bias voltage many times.

In an accelerated CT, however, the plasma does not remain stable long enough to sweep the bias voltage across any appreciable range. The triple Langmuir probe, although less accurate than a single probe configuration [34], has the benefit that it can instantaneously measure $I_{s a t}$ and $T_{e}$ [47], enabling a post processing analysis of an instantaneous density. Figure 4.6 [12] shows a basic schematic of a triple probe.

Three probe tips are arranged adjacent to each other, such that they are close 


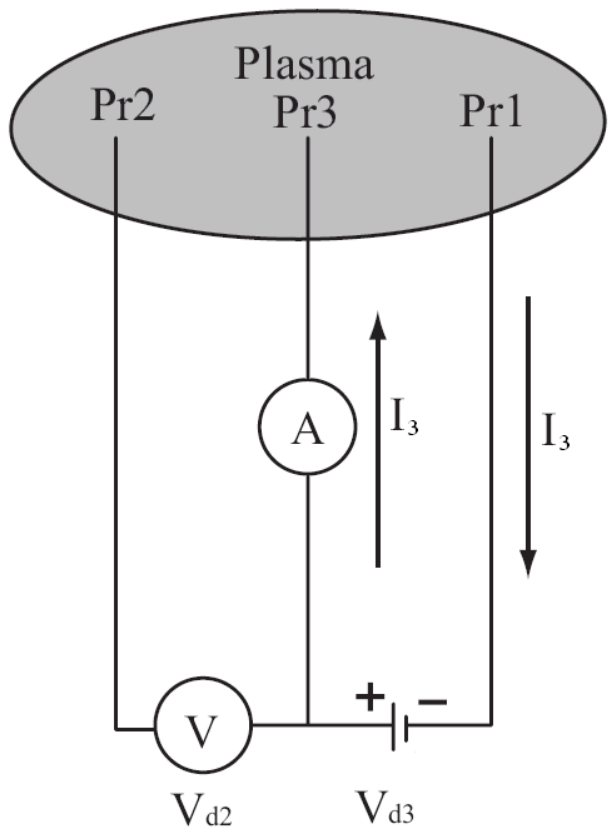

Figure 4.6: A basic triple probe circuit.

enough to measure the same region of plasma, but separated by 5 - 10 Debye lengths, so that their sheaths do not overlap. A constant voltage, $V_{d 3}$, is applied between two of the three adjacent probes, $\operatorname{Pr} 1$ and $\operatorname{Pr} 3$ in this case. Pr2 is left floating and the potential difference between $\operatorname{Pr} 2$ and $\operatorname{Pr} 3$ is $V_{d 2}$. If all the probes have the same size, the current measured through each of these probes can be described as

$$
\begin{aligned}
I_{3} & =-I_{e 0} e^{\frac{e V_{1}}{T_{e}}}+I_{s a t}, \\
0 & =-I_{e 0} e^{\frac{e V_{2}}{T_{e}}}+I_{s a t}, \\
-I_{3} & =-I_{e 0} e^{\frac{e V_{3}}{T_{e}}}+I_{s a t},
\end{aligned}
$$

where $V_{3}-V_{1}=V_{d 3}$ and $V_{d 2}=V_{3}-V_{2}$.

Solving with respect to $I_{\text {sat }}$ and $T_{e}$ it is found that

$$
\frac{1}{2}=\frac{1-e^{-e V_{d 2} / T_{e}}}{1-e^{-e V_{d 3} / T_{e}}}
$$




$$
I_{s a t}=I_{3} \frac{e^{-e V_{d 2} / T_{e}}}{1-e^{-e V_{d 2} / T_{e}}}
$$

The bias voltage, $V_{d 3}$ must be chosen such that $T_{e} \ll V_{d 3}$, normally by a few multiples. In such a situation, equations 4.22 and 4.23 reduce to

$$
\begin{aligned}
T_{e} & =\frac{e V_{d 2}}{\ln 2}, \\
I_{\text {sat }} & =I_{3} .
\end{aligned}
$$

When $V_{d 2}$ and $I_{1}$ are continuously monitored, a time evolution of electron density can then be calculated from equation 4.13 as

$$
n_{0}=\frac{I_{1} e^{\frac{1}{2}}}{q_{e} A \sqrt{\frac{k T_{e}}{m_{i}}}} .
$$

Figure 4.7, modified from [12] gives a schematic of the actual triple probe circuitry used on USCTI.

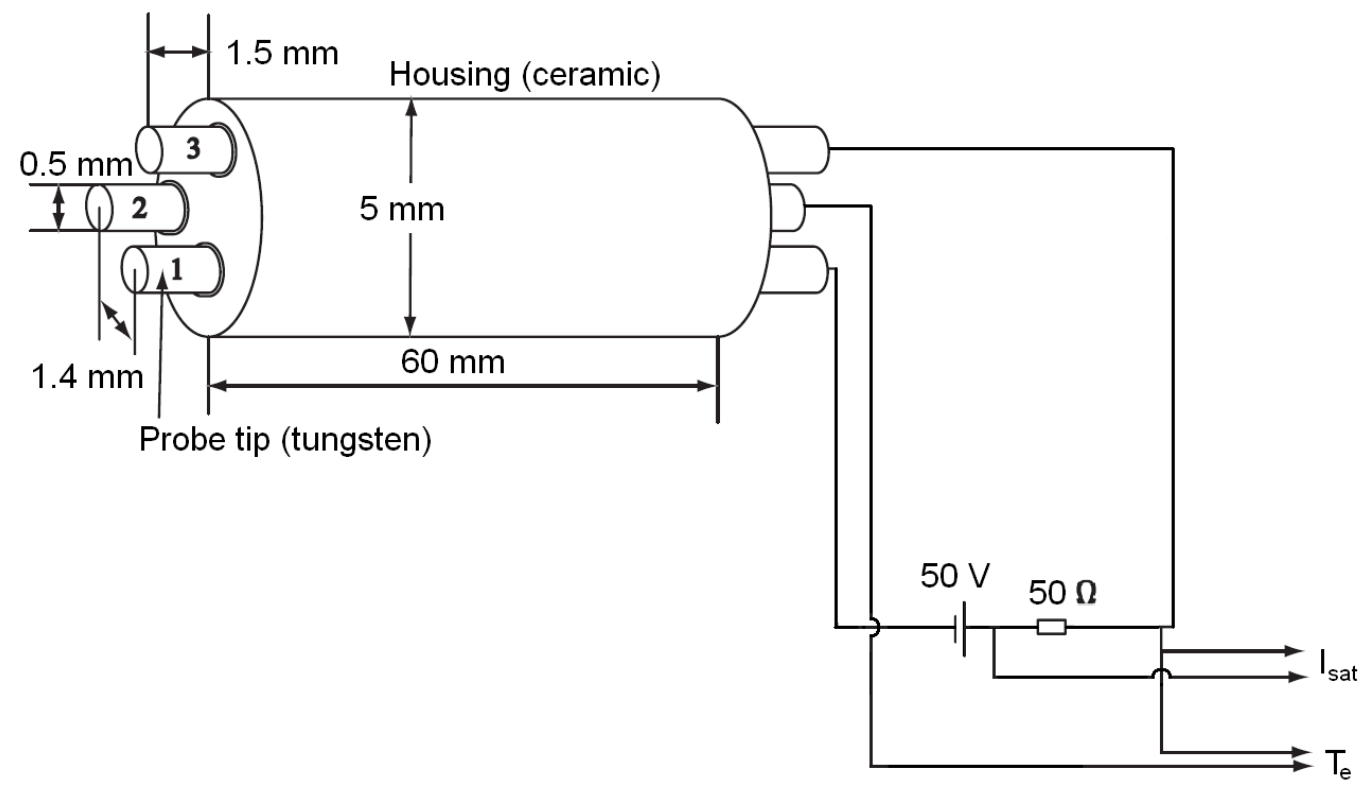

Figure 4.7: Diagram showing the arrangement of the triple probe used on USCTI. 
Instead of measuring the current directly, it was indirectly measured through $I=V / R$, where $V=V_{d 2}$ is the measured quantity, and $R=50 \Omega$.

In order to acquire the best signals, the probe was inserted at the magnetic axis, $r=0.63 R_{f c}$, where $R_{f c}$ is, in this case, the outer radius of the $\mathrm{CT}$. This is the region of the plasma with greatest density (similar to the tokamak core-region).

\subsubsection{Interferometry}

Another commonly used method for determining density in plasmas is interferometry. Interferometry measurements differ from Langmuir probe measurements in that instead of measuring the density at a single point, a line averaged density is calculated.

In the general sense, interferometry works by measuring the phase shift of an electromagnetic wave traveling through a plasma. The wave's frequency, $\omega$, must be larger than the plasma frequency,

$$
\omega_{p}=\sqrt{\frac{n_{e} e^{2}}{m_{e} \epsilon_{0}}},
$$

where $\omega$ has a dispersion relation $\omega^{2}=\omega_{p}^{2}+k^{2} c^{2}$. A typical value of $\omega_{p}$ for the USCTI plasma is $1.78 \times 10^{12} \mathrm{rad} / \mathrm{s}$.

The phase shift is measured by comparing the phases of two arms of the laser beam (see Figure 4.8). One arm travels though the plasma, and becomes shifted, the other is a reference beam, which travels an equivalent distance, but not through the plasma.

$$
\begin{aligned}
\Delta \phi_{p} & =\int_{0}^{L}\left(k_{0}-k\right) d x, \\
\Delta \phi_{p} & =\frac{\omega}{c} \frac{\omega_{p}^{2}}{2 \omega^{2}} L,
\end{aligned}
$$

where $\mathrm{L}$ is the distance traveled through the CT by the laser. By inserting Equation 4.27 into 4.28 and assuming $\omega \gg \omega_{p}$, we can easily find the line averaged electron density as

$$
n_{e}=\frac{2 \Delta \phi_{p} \omega c m_{e} \epsilon_{0}}{L e^{2}}
$$


or more specifically, for a He-Ne laser with a wavelength $\lambda_{0}=632 \mathrm{~nm}$,

$$
n_{e} \simeq 5.7 \times 10^{20} \frac{\Delta \phi}{L}
$$

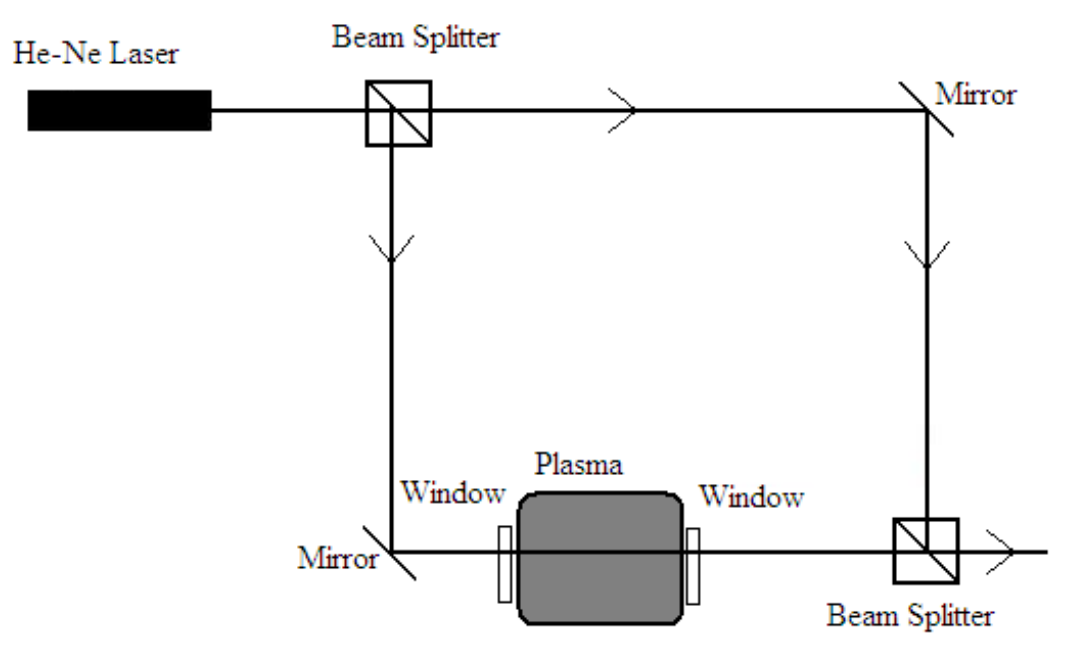

Figure 4.8: Basic diagram of interferometer used on USCTI.

Interferometry was used previously on USCTI, $[12,26,30]$ however was unable to be used in this project due to the arrangement of USCTI and STOR-M. When USCTI is connected to STOR-M, the interferometry port, located at the probe position z $=65 \mathrm{~cm}$, becomes covered by the bellows section of the vacuum chamber. Because USCTI relied on the vacuum pump of STOR-M, the devices were unable to be disconnected. USCTI's own pump was having problems (see Section 3.1.2) during operation, and could not be used.

\subsection{Data Acquisition and Analysis}

The triple Langmuir probe, used in the project, must be made to electrically float while in the plasma. Because the probe's measurements are taken with respect to itself, it can not be referenced to ground. The connection on the digitizer, however, is connected to ground. In order to acquire signals from the triple probe, the signals 
were passed through an isolation amplifier. A readily available, Tektronix A6902B, isolation amplifier was used in order to monitor the two Langmuir probe outputs $\left(I_{\text {sat }}\right.$ and $\left.V_{d 2}\right)$.

Data from the probes used was stored real-time using National Instruments (NI) software as well as hardware. An NI PXI-5105 digitizer was used to digitize the analog signals from the probes and transfer them to a computer. The PXI-5105 was chosen because of its fast sampling rate, $60 \mathrm{MS} / \mathrm{s}$, which was necessary since the CT moved through the observation location within $10^{-5} \mathrm{~s}$. It also has 8 input channels, which were needed for simultaneous data acquisition of all the magnetic and Langmuir probes. Data from the digitizer was saved to the computer using NI Labview, due to its ease of integration with NI hardware. Each shot was saved to a unique file, arranged in a matrix containing one row for each channel used.

Although the digitizer was capable of higher, the sampling rate used was 20 $\mathrm{MS} / \mathrm{s}$. This rate was found to give an adequate number of samples per shot without making post processing inefficient with regards to computation time. The maximum possible rate of firing (determined by the electrical system) was $1 \mathrm{~Hz}$. In order to assure that the computer could save data fast enough the digitizer was tested at its maximum sampling rate with all 8 channels set to acquire 2000 data points. The computer was found to be able to save data at upwards of $100 \mathrm{~Hz}$, far greater than what was needed for the experiments. This meant that each shot could be saved realtime, without the need for complicated acquisition procedures.

The files stored using Labview were then analyzed using the MATLAB software package, which is known for its signal processing capabilities. In order to determine repeatability of repetitive firing, correlation coefficients were calculated for each channel, for each shot, comparing them to a calculated mean of each channel. The mean calculated was

$$
\bar{x}(t)=\frac{1}{n} \sum_{i=1}^{n} x_{i}(t),
$$

the arithmetic mean of $n$ shots.

The correlation of the $i$ th shot, $x_{i}(t)$, to the signal mean, $\bar{x}(t)$, is calculated using MATLAB's built-in function, 


$$
\operatorname{corr}\left(x_{i}(t), \bar{x}(t)\right)=\frac{\sum x_{i}\left(t_{j}\right) \bar{x}\left(t_{j}\right)-\frac{\sum x_{i}\left(t_{j}\right) \sum \bar{x}\left(t_{j}\right)}{N}}{\sqrt{\left(\sum x_{i}\left(t_{j}\right)^{2}-\frac{\left(\sum x_{i}\left(t_{j}\right)\right)^{2}}{N}\right)\left(\sum \bar{x}\left(t_{j}\right)^{2}-\frac{\left(\sum \bar{x}\left(t_{j}\right)\right)^{2}}{N}\right)}},
$$

where the summation is performed over all data points at discreet times, $t_{j}$.

This provides a single value, the correlation coefficient, which is a measure of the linear relationship between the mean shot and the sample shot. As can be seen from the above equation, in the case where the two signals are exactly similar (meaning a complete linear relationship), the correlation coefficient would be exactly 1 .

By studying any time variations in the correlation coefficients of the data signals, any cumulative errors are able to be detected. This, in turn, demonstrates the repeatability of the $\mathrm{CT}$ production. In order to provide the most accurate correlation data, any inconsistent or failed shot's data is removed from the mean used in the correlation. If poor quality CT data was used to calculate the correlation, then the resulting coefficients would not be an accurate representation of the repeatability of the experiments. For example, if the CT failed to fire for one shot, it would be improper to use the respective data acquired from that shot to calculate the mean. This means that the correlation coefficients given in Chapter 5 are relative to the best case mean. An example of such a situation is provided in 4.9, which presents the mean shot from a series of data shots (shots 992 - 1091) as well as an example of a good shot (shot 994, with correlation coefficient $\sim 0.98$ ) and a bad shot which was excluded for the mean calculations (shot 1008, with correlation coefficient $\sim 0.58$ ).

Due to the nature of the probe signals, much of the data contained noise. RC integrators were used on the acquisition input line so as to filter out this noise. The signals from the Langmuir probes, however, were found to contain exceptionally high noise, and so for ease of operation, digital averaging was performed during post-processing. Although ideally it is best to perform such averaging real time, in MATLAB, a moving average can easily be preformed to any specified number of data points for fine tuning of the raw data.

In order to calculate the CT velocity, as shown in Equation 4.3, the time-of-flight for the $\mathrm{CT}$ at each probe was found by finding the peak magnitude of the probe 


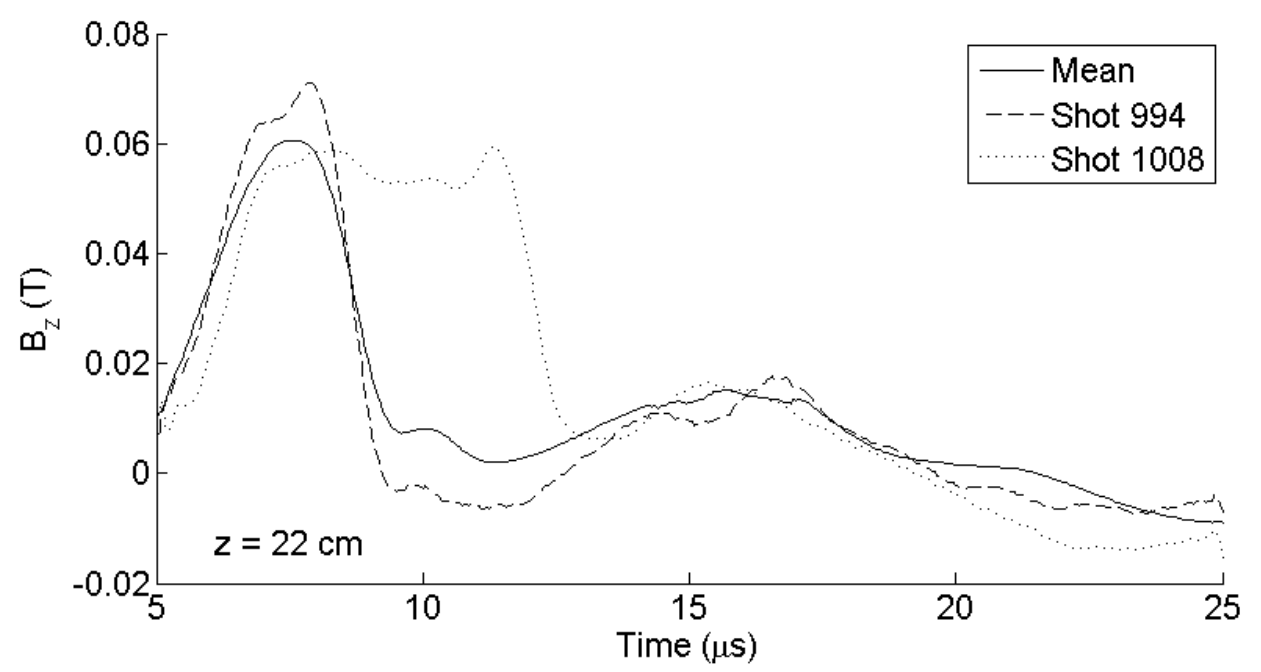

Figure 4.9: An example of a mean shot from poloidal magnetic field, $B_{z}$, measurements as well as a well correlated sample shot, 994 (with a correlation coefficient of $\sim 0.98$ ) and a poorly correlated shot, 1008 (with a correlation coefficient of $\sim 0.58$ ) which have been excluded in calculations of the best case mean.

signal using built in MATLAB functions. This proved difficult, however, to obtain accurate results, as even the slightest change in any smoothing function used on the signal would result in drastic changes of the velocity measurements. More on the data acquired is discussed in Chapter 5.

\subsection{Summary}

This chapter presented the diagnostics of CT plasmas as well as those used on the USCTI injector. An overview of magnetic probes, Langmuir probes and interferometry was given. A discussion of the methods and tools used for data analysis was also presented. 


\section{Chapter 5}

\section{EXPERIMENTAL RESULTS}

This chapter presents details of the results from experiments of repetitive operation of the USCTI accelerator. Examples of typical data collected are presented from all diagnostics used. Graphs of data correlation taken from successive shots are also given.

Experimentation for repetitive firing of USCTI was performed incrementally. Data presented consists of firing rates of once every 20 seconds, 15, 5, 4 and finally 3 seconds. The primary reason for this incremental experimentation was to avoid putting excessive stress on the system. USCTI was not originally intended for high repetition, and it was not known what components would be negatively affected. Simply firing the injector at the maximum limit for repetition would possibly bring to light too many unknown problems in the system at once. Limitation in repetition rate would not be discernable from other, more general problems, such as the grounding issues discussed in Section 3.2.2.

The diagnostics consist of four magnetic probes which were used to measure the $B_{z}$ magnetic field as the $\mathrm{CT}$ traveled through the injector as well as a triple Langmuir probe at the exit of the injector, to measure the final density of the CT (more on the diagnostics is presented in 4.1). The time of $t=0$ on the graphs corresponds to the time when the formation bank was fired. Since it took more than $5 \mu$ s for the $\mathrm{CT}$ to form and to be pushed to the first probe location, the first $5 \mu \mathrm{s}$ of data does not contain any useful data. The first $5 \mu$ s of data was thus omitted due to the noise appearing from the discharging formation and acceleration banks. The omission of this region mitigates discrepancies that would arise in the correlation between shots. 


\subsection{Preliminary Results}

This section contains acquired data on firing performed at 20 second intervals. This provides a base for which to compare higher repetition rates, as well as sort out any major issues with the injector.

Figure 5.1 shows sample data, shot numbers 576 and 581, from magnetic probes in a series of 11 repetitive firings. The voltage for the formation and acceleration capacitor banks used was $15 \mathrm{kV}$ and $10 \mathrm{kV}$, respectively. The magnitude of the poloidal $B_{z}$ field is shown at the 4 probe locations and is clearly pronounced. It is also clear to see how velocity data can be extracted from the figure. From the $\mathrm{z}=$ 43 and $65 \mathrm{~cm}$ probes, CTs produced in these shots have an approximate velocity of $v \sim 220 \mathrm{~km} / \mathrm{s}$, traveling $22 \mathrm{~cm}$ in $\sim 1 \mu \mathrm{s}$.
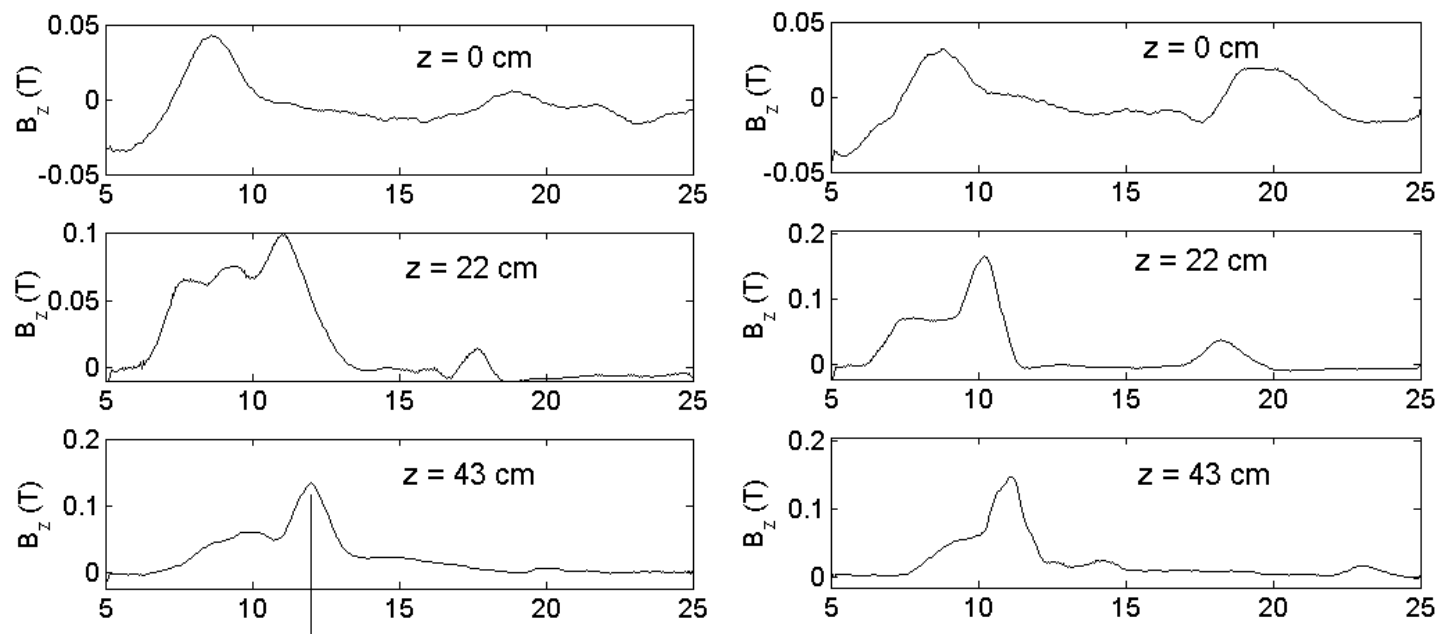

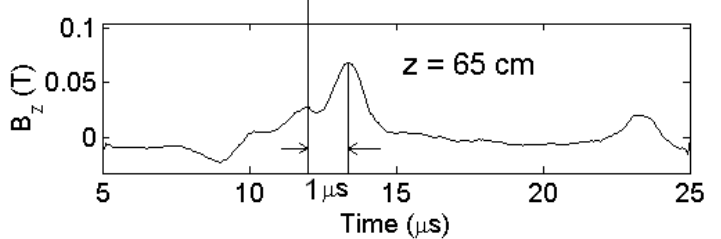

a)

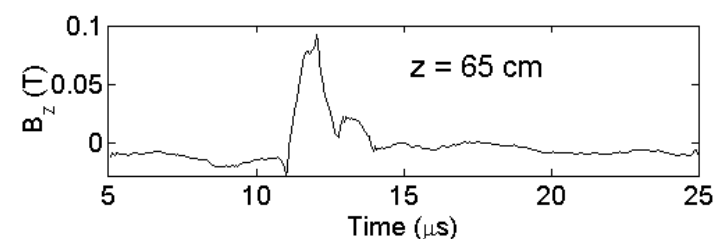

b)

Figure 5.1: $B_{z}$ probe data from shot numbers a) 576 and b) 581, in a 20 second interval firing. The time-of-flight of the CT in shot number 576 as it passes the $\mathrm{z}=43 \mathrm{~cm}$ and $\mathrm{z}=65 \mathrm{~cm}$ probes is shown.

Figure 5.2 gives an example of the mean signal from each probe in the same 20 
second interval firing set as well as the correlation coefficients (corr) of each firing with respect to its mean. Although not constant, the data signals are found to be well correlated with their mean. Shot number 581 gives the least consistent results, but it is clear from viewing Figure 5.1 that even a seemingly low correlation coefficient (corr), such as that from shot 581 produces a good quality CT. The sharp peak visible on the graph of magnetic probe data indicates a well formed CT. This is indicative that the injection on a 20 second interval is repeatable over 11 shots.
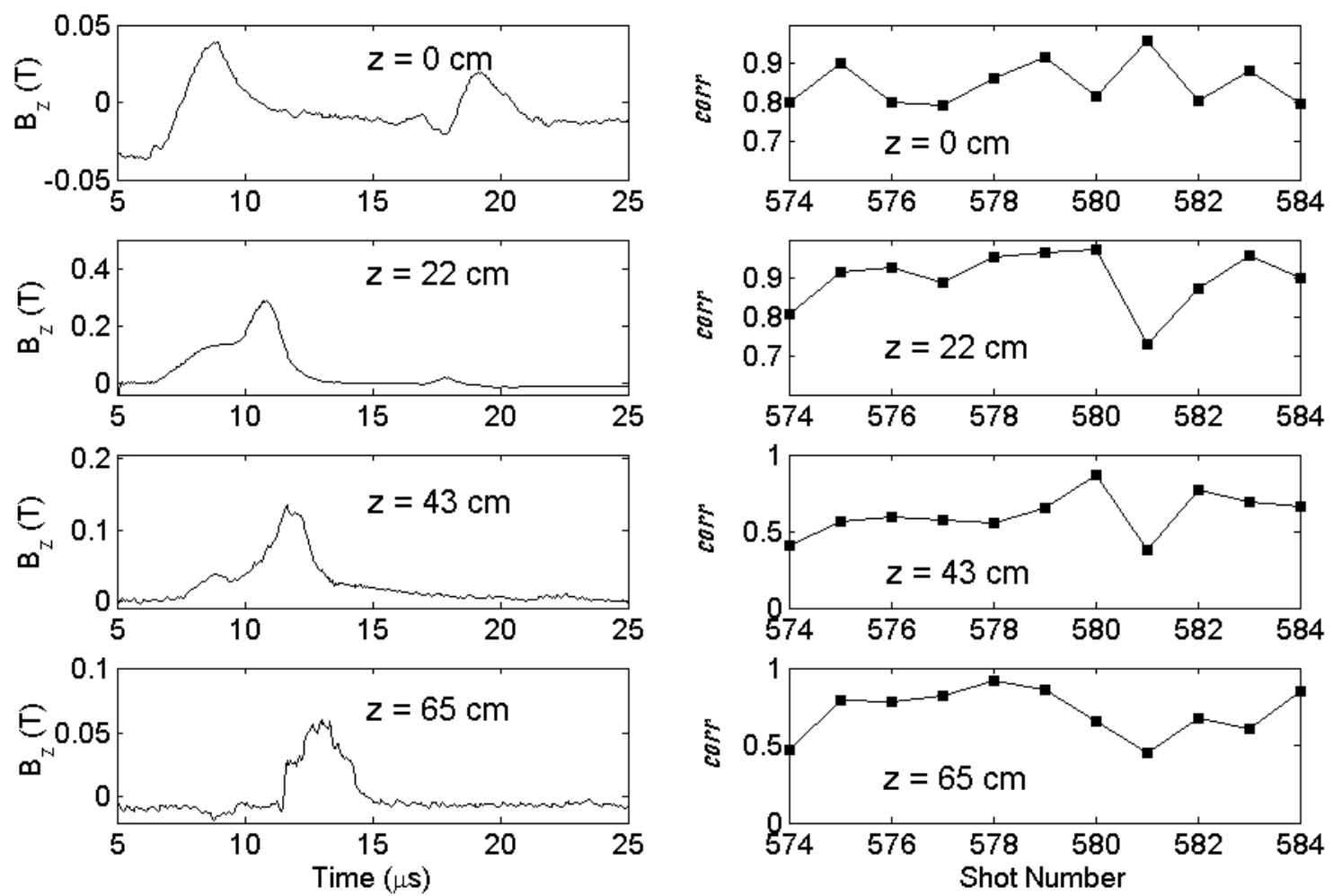

a)

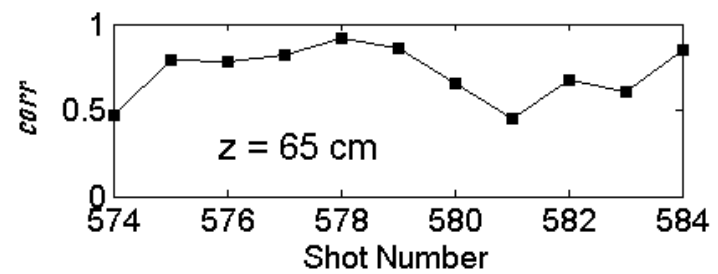

b)

Figure 5.2: a) mean $B_{z}$ probe data from shot numbers 574 - 584 as well as b) correlation coefficients of each shot with respect to their mean.

Tests were also conducted to determine any heating effects in the solenoid. Because the solenoid was completely enclosed within the injector, in order to determine its temperature, its resistance, $R_{\text {sol }}=0.4 \Omega$, was measured using an ohmeter. Knowing the dimensions of the solenoid's copper wire (cross-sectional area, $A=0.01 \mathrm{~cm}^{2}$, 
and length, $L=26.2 \mathrm{~m}$ ) its resistivity was calculated from

$$
\rho=R_{\text {sol }} \frac{A}{L}
$$

and compared to temperature-resistivity graphs for copper from Handbook of Chemistry and Physics [48]. The resistivity of the solenoid before firing was found to be $\rho$ $\simeq 1.7 \times 10^{-8} \Omega \mathrm{m}$ which correlated to a temperature of $\simeq 22^{\circ} \mathrm{C}$. Because the device had been sitting in room temperature for an appreciable amount of time (three days), a $22{ }^{\circ} \mathrm{C}$ initial temperature was expected. No change in resistance was observed after firing.

The minimum observable increase in temperature can be found by combining Equation 5.1 with the temperature and resistivity relation,

$$
\rho=\rho_{0}\left(1+\alpha\left(T-T_{0}\right)\right)
$$

where $\rho_{0}$ is the resistivity at initial temperature $T_{0}, \rho$ is the resistivity at final temperature $T$ and $\alpha$ is the temperature coefficient of resistivity, which for copper in the

temperature range considered in the experiment (i.e. $0-100{ }^{\circ} \mathrm{C}$ ) is $4.3 \times 10^{-3} \mathrm{~K}^{-1}$, and solving for $\Delta T$ as

$$
\Delta T=T-T_{0}=\frac{R_{\text {sol }} A / L-\rho_{0}}{\alpha \rho_{0}} .
$$

The minimum resistance change observable on the ohmeter used was $\Delta \mathrm{R}=0.1 \Omega$. This would correspond to an $R_{\text {sol }}$ value for Equation 5.3 of $0.5 \Omega$, corresponding to a $\sim 30^{\circ} \mathrm{C}$ increase in the solenoid's temperature. Furthermore, a temperature increase of this magnitude (though not observed) would not be expected to have an effect on the CT injector. Although baking was not being performed during the experiment, USCTI is typically baked (to mitigate outgassing effects) to a temperature of $60{ }^{\circ} \mathrm{C}$ - $100{ }^{\circ} \mathrm{C}$, without affecting the solenoid.

\subsection{Long Term Repetition}

An experiment set of 15 second intervals fired for 100 shots was conducted to determine longer term repeatability of the repetitive CT. The 15 second interval was 
chosen because the krytrons' power supplies being used were not able to reliably charge the krytron capacitors fast enough at shorter repetition rates. As well, this repetition rate avoids overloading of the vacuum system from by CT gas puffing. Until the krytron power supplies were replaced, the 15 second interval was the fastest firing frequency attainable with good results. The experiment was performed with a formation voltage of $V_{\text {form }}=18 \mathrm{kV}$ and an acceleration voltage of $V_{\text {accel }}=15 \mathrm{kV}$. These voltages for USCTI are the maximum values typically used during injection into STOR-M. For that reason, the results of this long term repetition provide a realistic case of CT injection. The shot numbers for the series were 992 - 1091. Figure 5.3 shows a sample shot from the series, as well as the mean and correlation of all shots with the mean.

Unfortunately, the magnetic probe at the position $\mathrm{z}=43 \mathrm{~cm}$ was not performing well for this experiment, and collected only noise. The data is still presented here as the other signals gave good results, indicating a problem with the probe, and not the CT itself. Subsequently it was found that the integrator on the acquisition line had malfunctioned; it was later repaired.

There were several shots in this series where the acceleration capacitor bank failed to fire. In the $\mathrm{z}=65 \mathrm{~cm}$ probe data, these correspond to all correlation coefficients less than 0.5 , which is confirmed by the absence of the acceleration discharge current, most likely caused by the krytrons failing to fire. The most important feature in the correlation data is the fact that the correlation coefficients do not decrease systematically with the shot number. If repetition rate and duration were affecting the quality of the CT, then a gradual worsening of correlation (i.e. CT quality) would be expected. It is clear from the collected data that the CT remained stable throughout the experiment.

As an example of the quality of the formation and acceleration current, Figure 5.4 gives the mean results of the formation and acceleration current waveforms of the 992 - 1091 series and the respective correlation coefficients. It shows that the formation bank fired very consistently, explaining generally high coefficients of the magnetic probe signal at $\mathrm{z}=0 \mathrm{~cm}$ in the formation region (see Figure 5.3. Occasionally, 

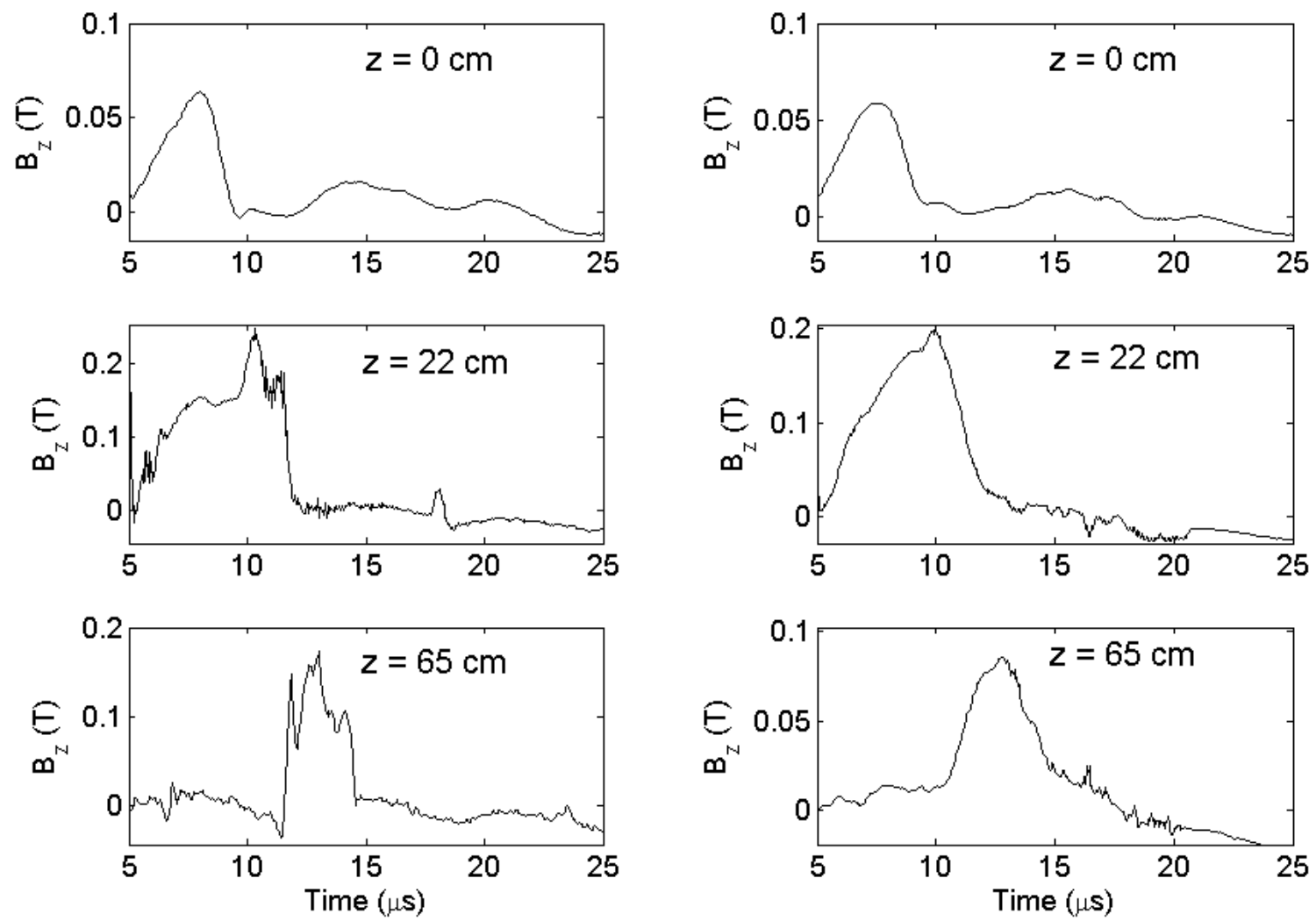

a)

b)
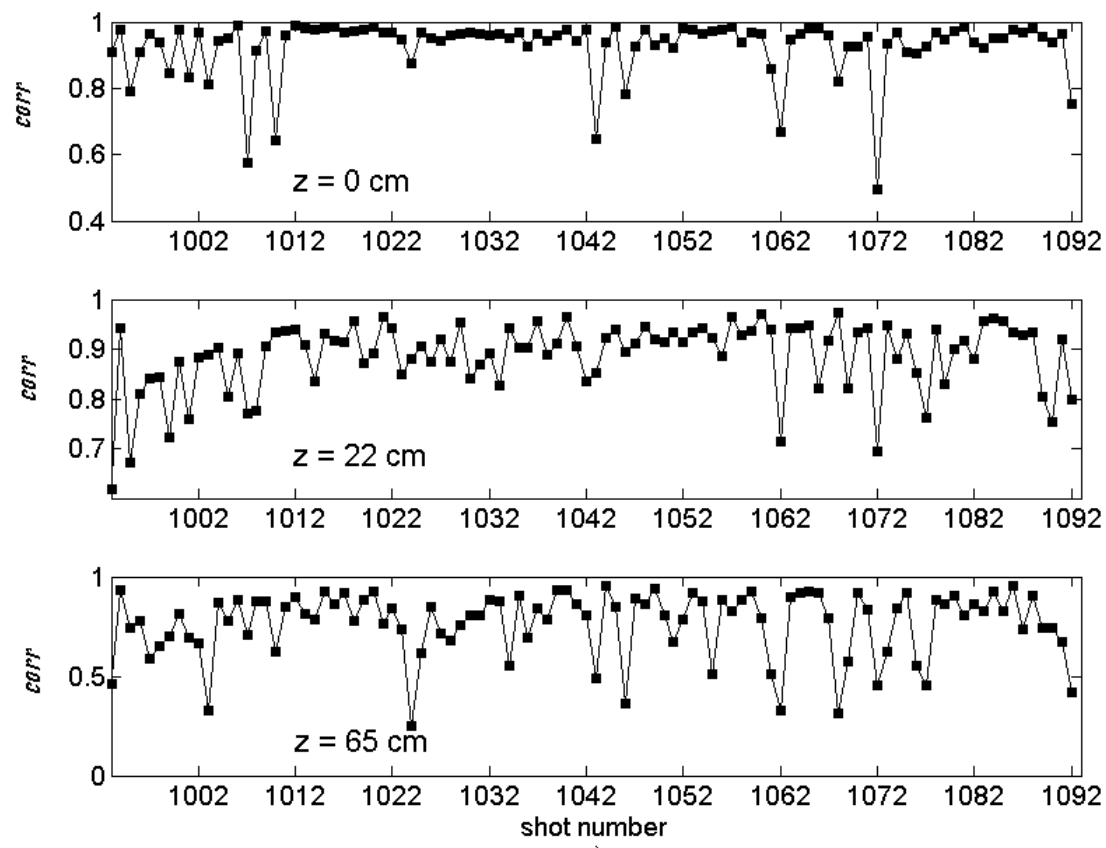

c)

Figure 5.3: Shot numbers 992 - 1091, a) shot 1021 and B) mean $B_{z}$ probe data as well as c) correlation coefficients of each shot with respect to their mean. 
low correlation coefficients appear for the magnetic probe data at $\mathrm{z}=0 \mathrm{~cm}$ (see Figure 5.3. These inconsistencies most likely correspond to the gas valve performance during these shots. Although there is a trend in the formation current's correlation coefficients, it occurs on such a small scale $(<1 \%)$ that it can not be taken as evidence that the formation bank is loosing its effectiveness
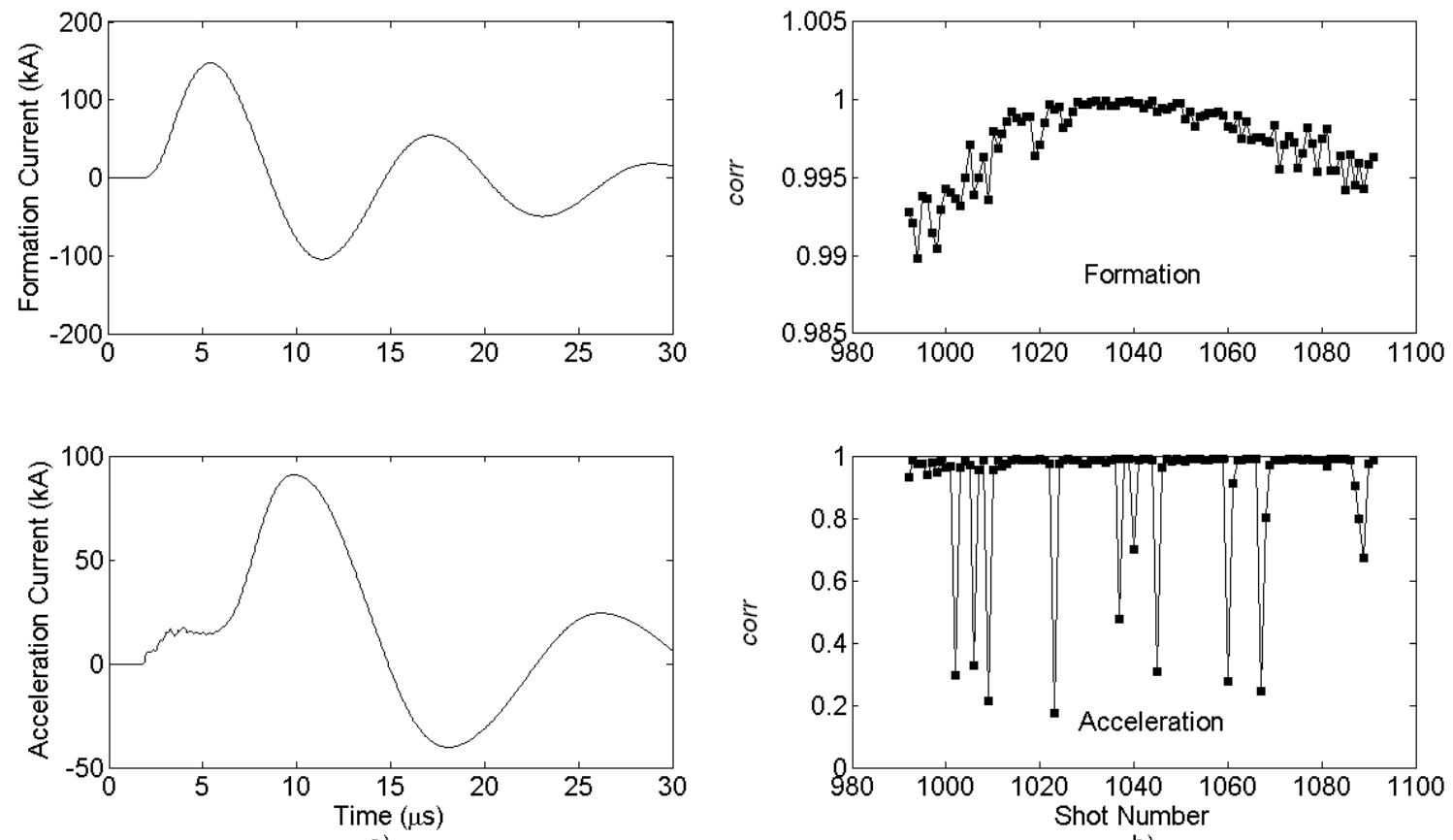

a)

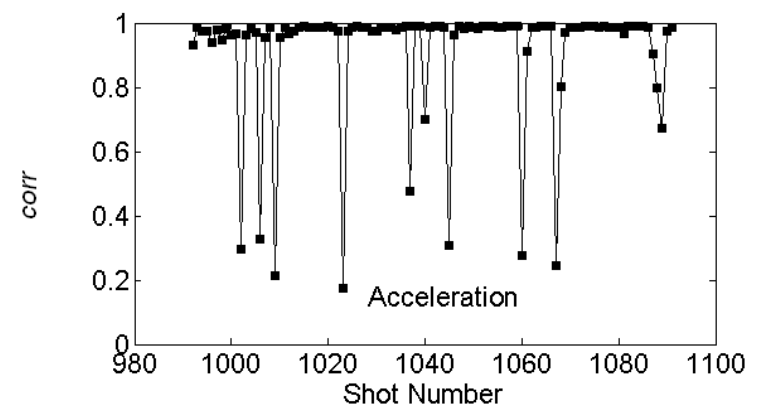

b)

Figure 5.4: Graphs of the a) mean and b) correlation coefficients of the formation and acceleration current waveforms for the 992-1091 shot series.

Shots in which the acceleration bank failed to fire are obvious from the data points with very low correlation coefficients in Figure 5.4. The most likely reason for this was because the krytron failed to trigger or mistriggered due to noise from the formation discharge. Since the acceleration krytron was older than the one used on the formation bank, it was found to have inconsistencies in performance.

Pressure in the vacuum chamber during the discharges was monitored using a Veeco pressure controller. It was found that the pressure stabilized between each of the 100 shots. No pressure buildup was observed. 
The temperature of USCTI's components was measured with an Infra Red temperature probe before and after the long term repetition, and no appreciable difference was observed. The gas valve solenoids, main gun assembly, and the gas valve and solenoid SCRs were all measured and found to have only a $2-3{ }^{\circ} \mathrm{C}$ increase in temperature. The formation solenoid also had no noticeable change in temperature. Because the repetition rate was relatively slow (once every 15 seconds) this result was expected.

\subsection{Density Measurements}

A triple Langmuir probe was used at the exit of the acceleration region, the position is best described as $\mathrm{z}=83 \mathrm{~cm}$, to calculate the CT final density. The triple Langmuir probe data proved to contain a large amount of noise, and although post processing filters were used to minimize the noise's effect, it is still evident in the data. Figure 5.5 shows a sample shot (1253) and the mean of a 19 shot series $(1237$ - 1255) in a 20 second repetitive mode. The formation voltage for the series was $V_{\text {form }}=17 \mathrm{kV}$ and the acceleration voltage was $V_{\text {accel }}=13.5 \mathrm{kV}$.

The early part of the data from Figure 5.5 has been removed because it does not contain useful data, but merely noise. The signals presented in $T_{e}$ and $I_{\text {sat }}$ are the two output signals from the probe, with Equation $4.24\left(T_{e}=e V_{d 2} / \ln 2\right)$ being applied to the output signal, such that $T_{e}$ is given in $\mathrm{eV}$. Density is calculated from these two signals as post processing.

To further show how the density data is related to the magnetic probe data from previous sections, Figure 5.6 shows the CT magnetic probe data along with the density from the shot series 1582 - 1591. The data presented is from a 10 shot series with an interval in firing of 5 seconds. The formation voltage for the series was $V_{\text {form }}$ $=17 \mathrm{kV}$ and the acceleration voltage was $V_{\text {accel }}=14 \mathrm{kV}$. These voltages were chosen because they are typical settings used for CT injection into STOR-M. They are less than the maximum typically used to avoid unnecessarily over-stressing the device's components. 

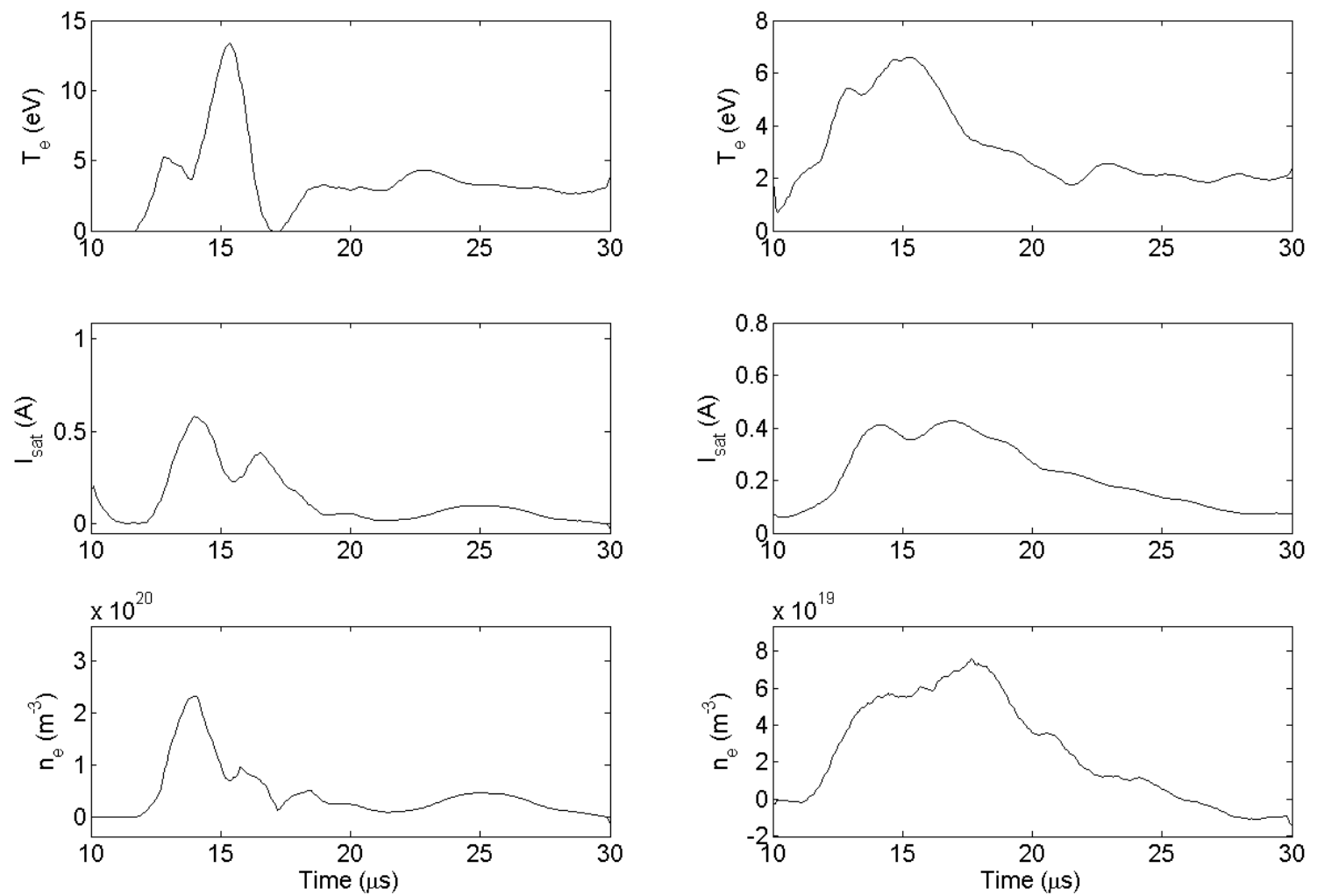

a)

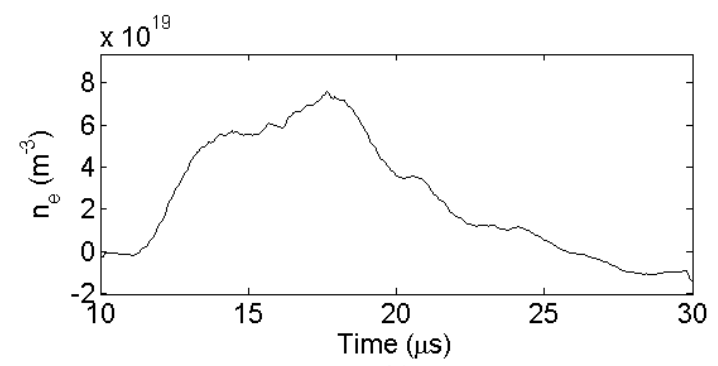

b)

Figure 5.5: Triple Langmuir probe data from preliminary density measurements. $T_{e}, I_{s a t}$ and $n_{e}$ are presented from a) shot 1253 and b) the mean from shots 1237 to 1255 . 

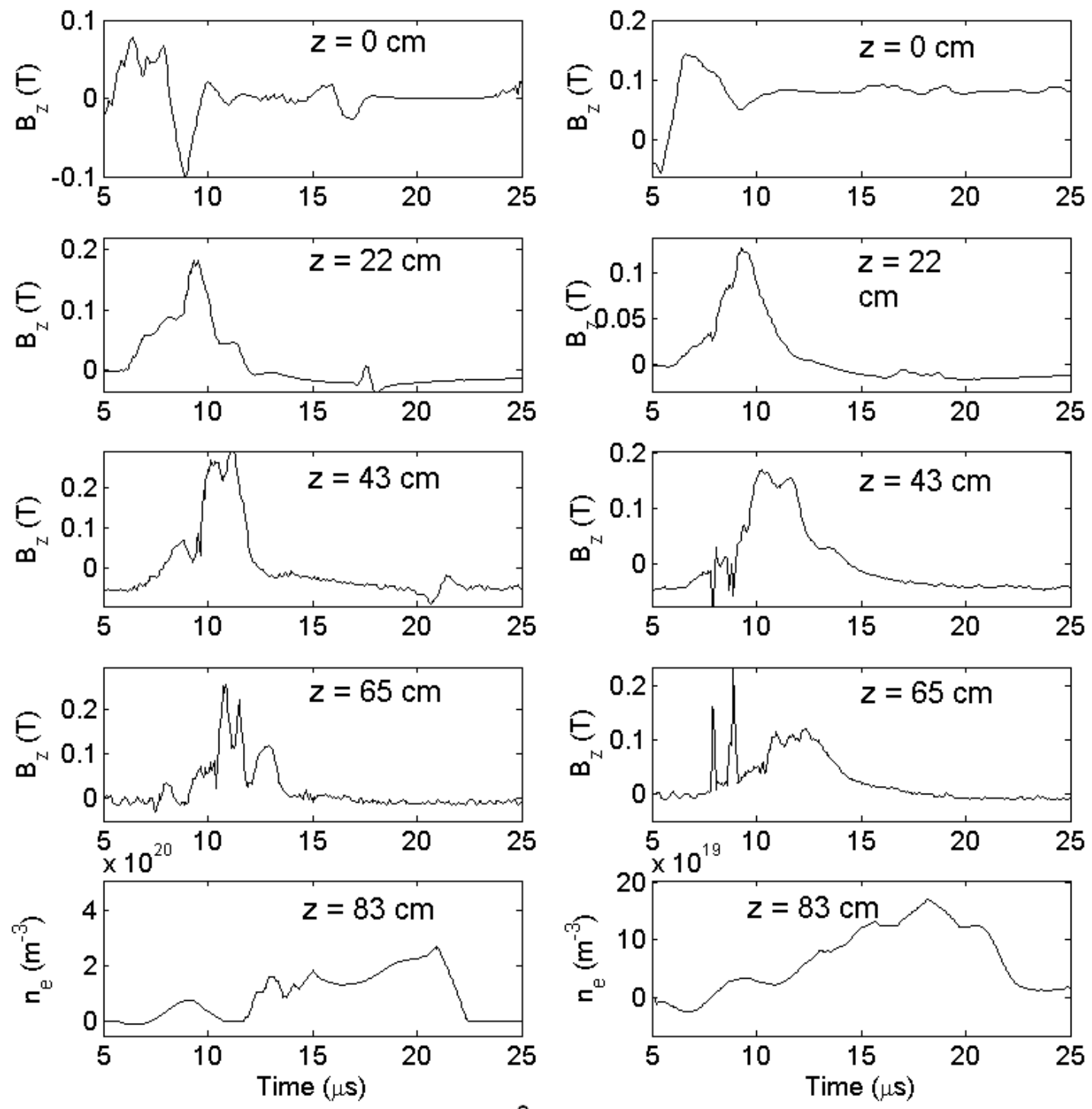

a)

b)

Figure 5.6: a) shot 1585 and b) mean $B_{z}$ and $n_{e}$ probe data from the 1582 - 1591 series. 
The noise present in the signal is evident, however the results are still acceptable. The CT reaches the Langmuir probe at a time consistent with the magnetic probe time-of-flight. Because the density data from both Figures 5.5 and 5.6 was consistent with a good quality CT, this relation is not unexpected, however it adds to the evidence of repeatable repetitive firings.

The peak density measured is on the order of $10^{20} \mathrm{~m}^{-3}$, lower than those previously measured using an interferometer $[12,26,30]$. This is likely due to the positioning of the density probe. The $n_{e}$ of $\sim 10^{21} \mathrm{~m}^{-3}$ was measured using an interferometer placed at the $\mathrm{z}=65 \mathrm{~cm}$ port, the triple probe used in this experiment was placed approximately $13 \mathrm{~cm}$ after the end of the acceleration electrodes.

When the CT leaves the acceleration region, it will begin to both decay and expand. From Equation (2.19) it can be found that the CT lifetime is on the order of $20 \mu \mathrm{s}$, so the decay time should not drastically affect the density measurements, although some decay would occur.

When the CT is no longer contained in the acceleration region, and passes through the focussing cone, it will be both compressed and elongated. This will create two factors that would have an effect on the density. The first is that the elongation will make the CT have a lower density overall. The fact that the CT is compressed means that the magnetic axis is not confined within the acceleration electrodes, and is more difficult to locate. This means that the magnetic axis would have moved radially inward. The probe was positioned such that this would be compensated for, however it is not exactly known by how much the CT's magnetic axis would be compressed inward, so the Langmuir probe's position relative to the magnetic axis is not exact. If the probe is not at the magnetic axis, the measured density would be lower than those at the magnetic axis.

What is most important, however, is the repeatability of the density measurements. The density measurements are consistent and show that CT production is repeatable. The slow decay of density as the CT passes the probe may be indicative of trailing plasma following the CT. This phenomenon is not revealed by the magnetic probes because they are measuring $B_{z}$, and the trailing plasma may not 
contain any $B_{z}$ component. Trailing plasma is a common phenomenon in CTs and other accelerated plasmas $[32,49]$.

\subsection{Higher Repetition Rate}

In the above experiments, the limiting factor was the krytron capacitor banks' charging time. When new power supplies were acquired, and the charging resistors were reduced in resistance, higher repetition rates became feasible. Figure 5.7 shows the results from a 4 second interval shot series. The series presented had a formation voltage of $V_{\text {form }}=17 \mathrm{kV}$ and an acceleration voltage of $V_{\text {accel }}=14 \mathrm{kV}$. The shot number ranges from 1615 - 1622 .

The highest achievable repetition rate for USCTI was $0.33 \mathrm{~Hz}$, or one shot every 3 seconds. Figure 5.8 depicts the results of one of the experiments performed with a 3 second interval. The bank voltages for the 3 second interval experiment were also $V_{\text {form }}=17 \mathrm{kV}$ and $V_{\text {accel }}=14 \mathrm{kV}$. The series is from $1640-1645$.

The correlation coefficients of both the 4 second and 3 second interval experiments are presented in Figure 5.9. As is evident from the data, the shots correlate well, and the correlation coefficients are comparable to lower frequency repetition experiments.

The observed repetitive firings for these high frequency experiments are shown to agree well with other data collected during the experiments with lower repetition rates. This shows that the CT firing is also repeatable for higher repetition rates. Comparing the graphs from the 3 second interval data and the 20 second interval data, it is clear that the CT structure remains constant. This comparability is key in recognizing good quality firings in higher frequency repetitions due to the nature of the experiments.

The high frequency $(0.33 \mathrm{~Hz})$ repetitive shots are rather undocumented for $\mathrm{CT}$ injectors. It was not known, at the commencement of the project, whether any of the shots would produce good quality or consistent CTs. The fact that the mean data as well as sample shots from the magnetic field and density from $0.33 \mathrm{~Hz}$ firing agree with lower frequency firings indicates that the CT is unaffected by the frequency of 

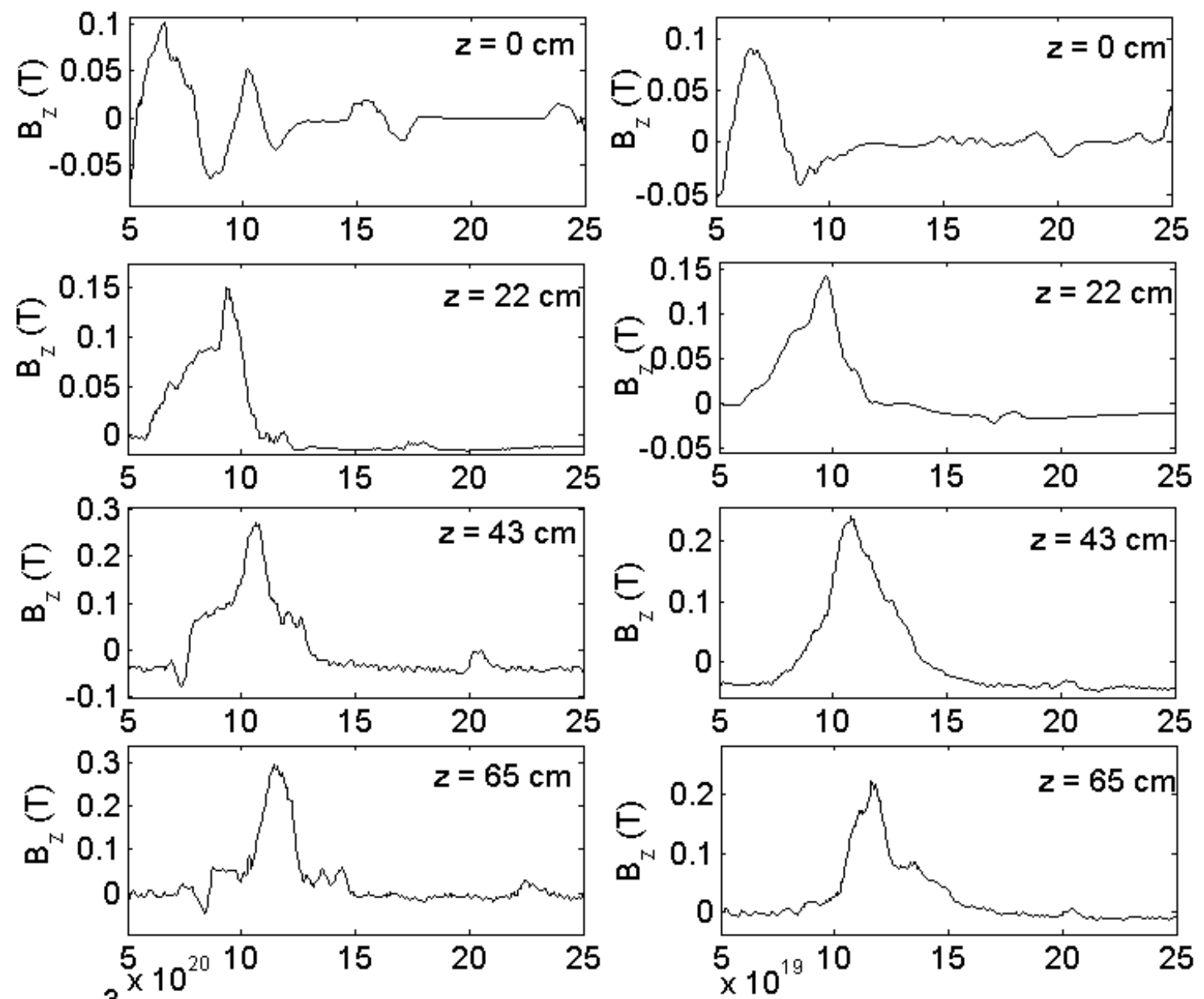

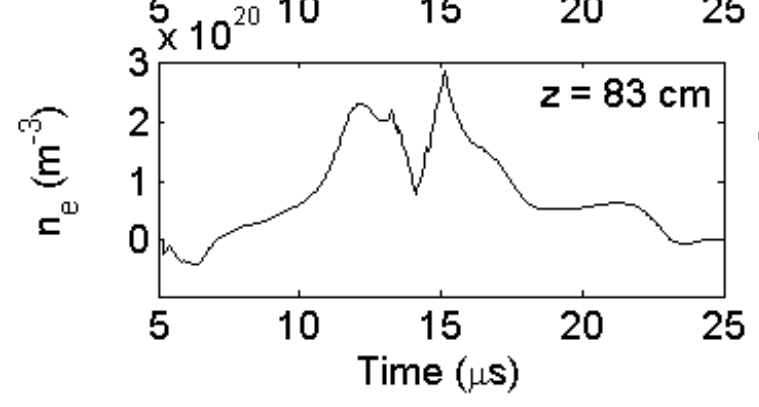

a)

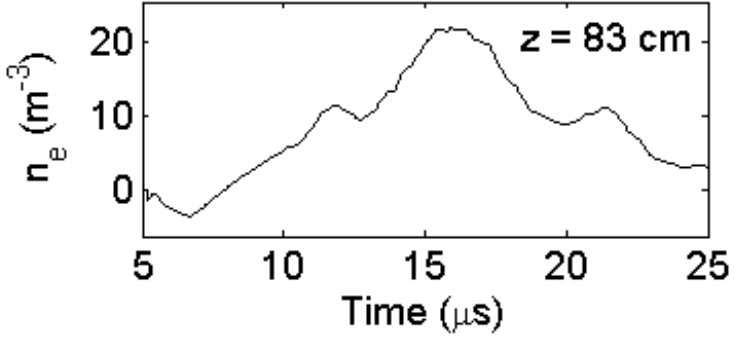

b)

Figure 5.7: 4 second interval data from shot 1615 to 1621 . a) a sample shot, 1616 and b) mean $B_{z}$ and $n_{e}$ probe data are presented. 

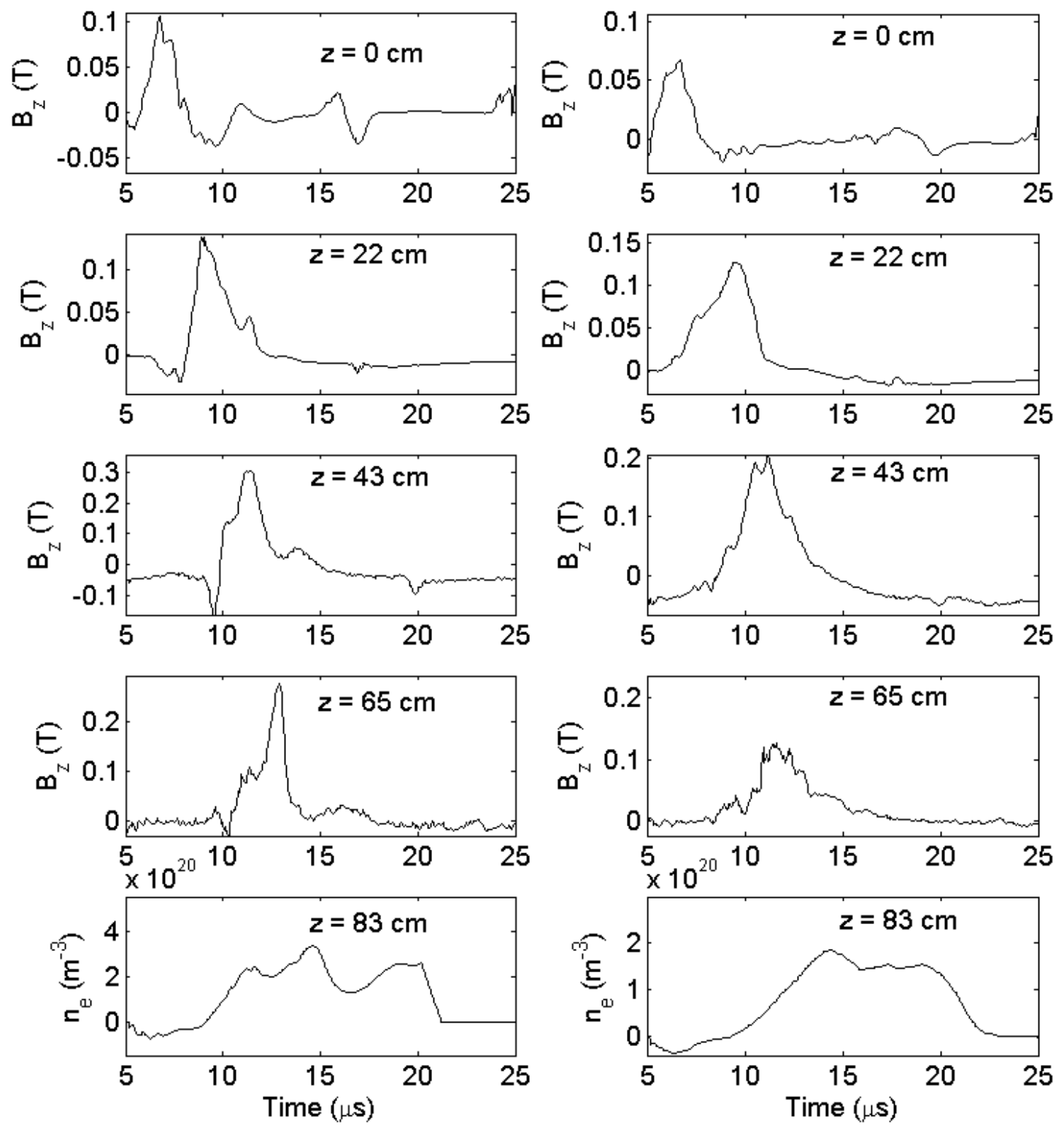

a)

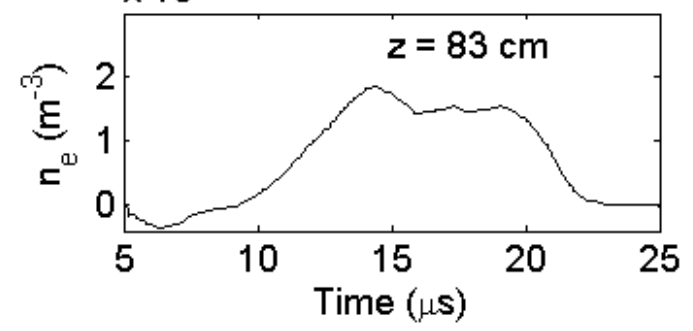

b)

Figure 5.8: 3 second interval data from shot 1640 to 1645 . A) a sample shot, 1641 and b) mean $B_{z}$ and $n_{e}$ probe data are presented. 

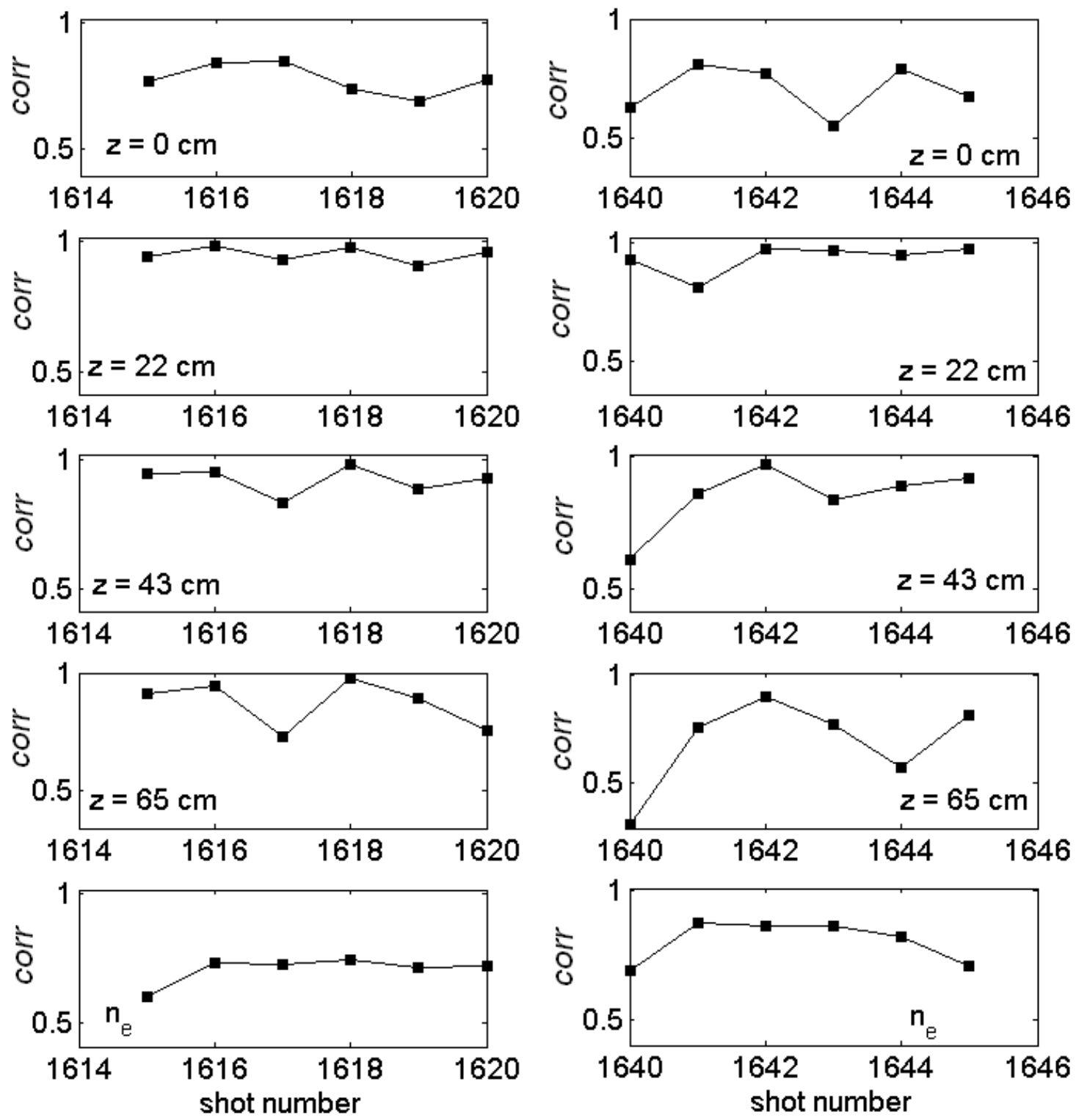

a)

b)

Figure 5.9: Correlation of high repetition measurements, a) 4 second and b) 3 second interval firings. 
repetition. This repeatability is an important factor in tokamak fueling.

\subsection{Time-of-Flight Analysis}

The velocity of the CT says a lot about its reproducibility. Theoretically, the CT's velocity is controlled by the power transferred to it via the acceleration discharge, which is in turn controlled by their charging voltage. If the CT is formed properly, then there should be no difference in its acceleration and velocity.

Figure 5.10 shows the velocities from the 3 second interval experiments discussed in Section 5.4. Although the velocities are not constant, it is clear they match well with the previously measured velocity, $v \sim 200 \mathrm{~km} / \mathrm{s}$, during single shot USCTI operation. The average velocity from these firings was $235 \mathrm{~km} / \mathrm{s}$. For these shots, the formation bank voltage, $V_{\text {form }}=17 \mathrm{kV}$ and the the acceleration bank voltage, $V_{\text {accel }}=14 \mathrm{kV}$. As previously mentioned, these are normal operating voltages for injection into the STOR-M tokamak.

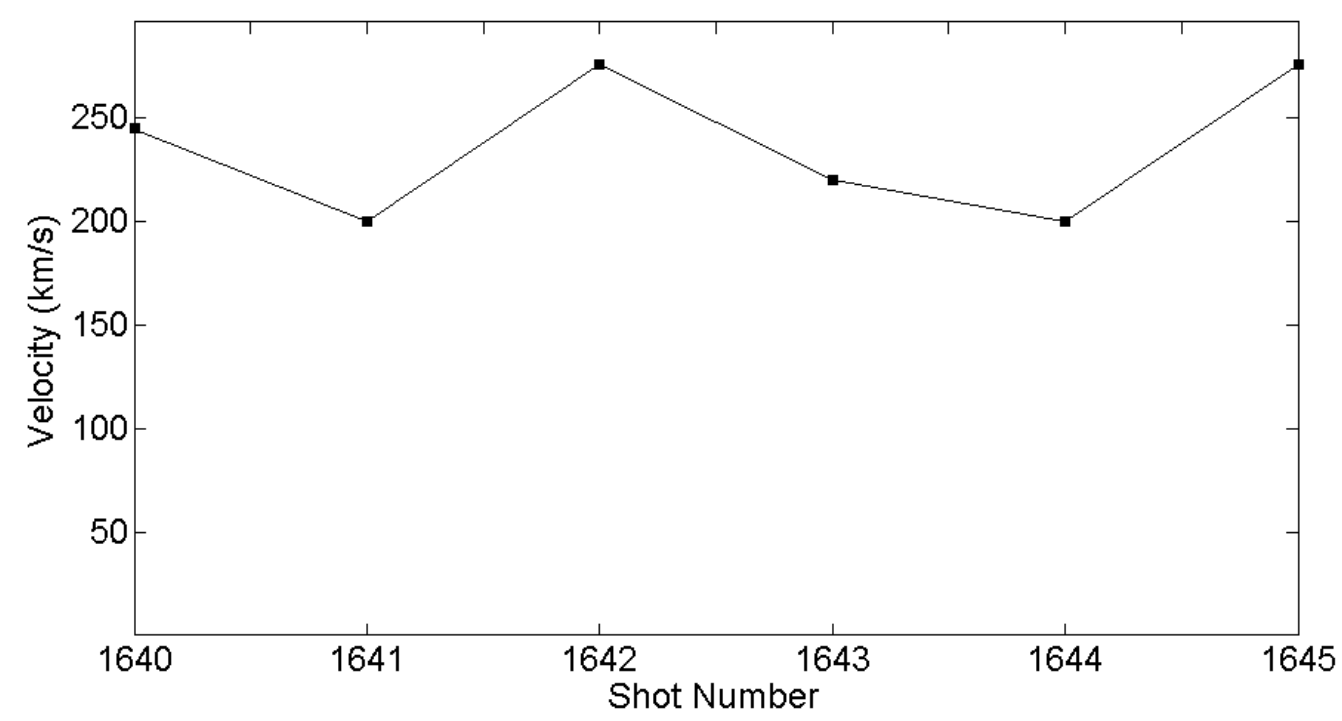

Figure 5.10: Velocities of the CTs from 3 second interval repetition experiments.

The inconsistencies in velocity measurements are due mainly to variations in 
smoothing of the raw data. As mentioned in Section 4.2, data was post-processed using moving averages of the data. It was found that small changes in the window size of the moving average led to significant changes in some of the calculated velocities. This effect of smoothing was a problem only because two different probes were compared to each other. It was not seen as a problem when correlating the data in repetitive shots, as in that situation each probe was only compared to itself. Any effect that smoothing would have on a probe's signal would be constant, so long as the signal was shown to be repeatable.

\subsection{Summary}

This chapter presented the results from repetitive experiments performed on USCTI. Typical CT parameters were presented and compared to the means from series of shots at various repetition rates. In order to determine repeatability of the CT firings, correlations between the individual shots and the mean of their respective series were presented.

It was shown that for the experiments performed, repetitive operation is feasible for USCTI. Data collected from higher repetition rates (3 second interval) matches well with data collected from lower repetition rates (20 second interval). Furthermore, it was shown that the data from high repetition rates correlates well to itself; the CT does not noticeably degrade in quality with successive firing. 


\section{Chapter 6}

\section{Conclusions And Future Work}

This project was performed in order to modify and test the University of Saskatchewan Compact Torus Injector in high repetition rates. This final Chapter presents a summary of the repetitive operation experiments performed on USCTI to date. The conclusions and a discussion of the experiments are given. A discussion of future work on USCTI as well as CT injectors in general is also presented.

\subsection{Summary and Conclusions}

CT injectors, in general, are promising to be effective at fueling tokamaks, and are essential for large scale, commercial implementation of fusion power using tokamaks. For complete viability, however, they must be able to continuously fuel a tokamak, and therefore be implemented in a repetitive mode.

Before this project, the best repetition rates were $\leq 0.2 \mathrm{~Hz}$, far below the upwards of $50 \mathrm{~Hz}$ necessary for commercial implementation. With experimentation on USCTI, repetitive operation of $0.33 \mathrm{~Hz}$, or 1 shot every 3 seconds, was achieved with good results.

Modifications made to USCTI in order to perform this repetitive operation mainly surrounded its capacitor banks. Their control system was altered and interlocks, designed to cycle the injector's power after each firing, were altered or bypassed. Newer, more powerful power supplies were acquired, which made charging times significantly shorter. Changes in the circuitry were made to enable fast firing. The grounding relays, that closed when firing was complete, were bypassed in order to avoid arcing and unnecessary cycling of the switches. 
Override switches were used on the formation and acceleration capacitor banks. These enable USCTI to be fired in both a repetitive mode, where grounding relays are not closed, as described above, or in a normal operation mode. During normal operation, when the CT is synchronized with STOR-M and remains unfired for minutes at a time, the risk of having high power, ungrounded capacitor banks was avoided.

Experiments were performed at various repetition rates in order to determine the various limitations of the injector in a step-by-step manner. This limited the number of potential problems to be dealt with at any one time. This proved to be helpful, as many issues developed throughout the experiment, some of which were unrelated to the frequency of repetition, such as the arcing in the Ross relays as well as grounding issues.

Data collected for determining repeatability included magnetic probe data, which measured the poloidal field, $B_{z}$ at four axial probe locations along the injector, $\mathrm{z}=0$ $\mathrm{cm}$, at the formation region, $\mathrm{z}=22,43$ and $65 \mathrm{~cm}$, along the acceleration region. The density of the CT upon exiting the injector was determined using a triple Langmuir probe. Using this type of probe, electron temperature and density were measured instantaneously.

In order to quantify the successfulness of the CT repetitions, probe data from each shot for a given repetitive firing series was correlated with the mean result from the series. Through looking at data in this way, any discrepancies in firing were able to be determined. Any systematic problems with CT firing would present itself in the data as a decrease, over time, in the correlation of the shots with respect to their mean.

It was shown, in Chapter 5, that the correlation coefficients of data at all repetition rates were high. Data obtained from the 20, 15, 10, 5, 4 and, most notably, 3 second repetition experiments showed the production of repeatable CTs. Data from the magnetic and density measurements shows that the CTs are repeatable within the scope of the project.

The magnitude of the poloidal magnetic fields, $B_{z} \simeq 0.2 \mathrm{~T}$, along the accel- 
eration region and $B_{z}=0.05 \mathrm{~T}$ in the formation region, are consistent with past experiments. Although the density measurements showed a low density, $\sim 10^{20} \mathrm{~m}^{-3}$, the measurements themselves were repeatable throughout the experiment. It was discussed in Section 5.3 that the low magnitude of the density may have been caused by the placement of the probe used, and not due to a low quality CT. Velocities of CTs in higher repetition experiments $(0.33 \mathrm{~Hz})$ were shown to agree with the necessary velocities $(\sim 200 \mathrm{~km} / \mathrm{s})$ for integration with STOR-M.

Particularly notable is the well correlated formation probe data, particularly in the long term repetitive measurements, indicating that any discrepancies occur within the acceleration region, the main reason for this, being problems with the krytrons that switched the ignitrons controlling the formation and acceleration capacitor banks.

Data from high repetition experiments $(0.33 \mathrm{~Hz})$ was shown to be consistent with lower repetition frequency experiments. Sample shots as well as mean data and correlation coefficients from high frequency experiments are indistinguishable from low frequency experiments. This is an important fact as it is indicative of a feasible mode of operation at the repetition rates explored.

\subsection{Future Work}

The work involved in this thesis has shown that integration of repetitive firing injectors for use in large scale tokamaks is not a question of how, but merely when. As discussed, other, presently implemented, fueling technologies are inadequate for future work on tokamaks. Compact torus injection is a viable candidate as a fueling method for tokamaks, and although there is always room for improvement, the next step for CT advancement is an engineering issue.

As explained in Section 3.2.1, when the formation and acceleration banks are discharged, a damped oscillation of current continues after the CT is formed and accelerated. In the future, a high current pulse forming circuit should be designed such that only a single positive current pulse is delivered to the CT. In this manner, 
the unneeded energy can be saved in the banks, and only the used energy will need to be replenished.

Ultimately, the future for CT injectors lies in their implementation with, not simply larger tokamaks, such as ITER, but with full scale, commercial reactor tokamaks. The work performed within this thesis has brought to light several issues which must be contended with in order to advance $\mathrm{CT}$ injection to this point.

Repetitive operation was not intrinsically built into the original USCTI plans. During operation, as discussed in Section 3.2, there seemed to be physical limitations to running of the injector. The largest issue lied in the gas valves. It was found that the coils in the gas valves arced to the CT body. This created a hole in the diaphragm of the valve and caused the hydrogen feed to leak to atmosphere. Fortunately it did not cause a leak into the vacuum chamber, however, when the gas valve broke in such a manner, it was not an easy fix. The chamber had to be brought to atmosphere, the valve removed, repaired, and then replaced. In most research settings, such periodic adjustments and maintenance are not of great concern. In a realistic commercial setting, however, lost time can be disastrous and costly, (for example, if residents are out of power). Problems that might seem trivial and can be easily overlooked in a research setting must be carefully studied and mitigated in an industrial setting.

Issues related to the krytrons were discussed throughout this thesis and although they are mostly specific to experiments with USCTI, they must be contended with. Since USCTI's construction, advancements have been made that would allow for newer, better technological implementations to replace the krytrons in order to further experiments with the present system at the University of Saskatchewan.

Although heating of the solenoid was not an issue on USCTI, it has been previously observed at UC Davis $[14,33]$ and is an understandable issue to be dealt with on large scale operations. Cooling systems must be developed to ensure consistent solenoid operation over long (i.e. indefinite) time periods. One possible way to cool the solenoid would be implement a pressurized, force flow, system with $\mathrm{SF}_{6}$ gas through the solenoid chamber.

It was shown that repetitive experiments are reproducible on USCTI. The task at 
hand now is to perfect the device to fire the best attainable quality CTs. Feasibility for repetitive operation has been shown, but the quality of fired CTs must be proven for injection into tokamak plasmas to be feasible. Although repetitive CT firing has been achieved, actual multiple CT injection into a tokamak discharge has yet to be experimented. If there is one area that must be further experimented, it is this fundamental application of repetitive CT injection that must be tested. As perfectly consistent as the $\mathrm{CT}$ can be measured to be upon exit of the accelerator, it must be shown, undoubtedly, that the multiple CTs are, in fact, properly fueling the tokamak plasma. These experiments would not be unprecedented, diagnostic and measurement tools used in original experiments, proving the fueling potential of CT injection (at TdeV, STOR-M, JFT-2M) would be sufficient to test multiple fueling injections on newer, larger, longer duration tokamaks.

Newer CT injectors must take these factors into consideration. It is clear from this project that the physics behind repetitive operation is viable. In order for repetitive operation to be feasible, it is not only the theoretical, but also the technical issues that must be dealt with. 


\section{REFERENCES}

[1] Ward, D. J., Cook, I., and Miller, R. L. Fusion Engineering and Design 63-64, 25-33 (2002).

[2] Zucchetti, M. Annals of Nuclear Energy 32, 1584-1593 (2005).

[3] Esham, B. D. Wikimedia Commons.

[4] JET. Nuclear Fusion 32, 187-203 (1992).

[5] Dolan, T. J. Fusion Research. Pergamon Press, (1982).

[6] Bruno, C., Lawrence, T. J., Fearn, D., Auweter-Kurtz, M., Kurtz, H., and andAlessio Del Rossi, R. X. L. Nuclear Space Power and Propulsion Systems, volume 225 of Progress in Astronautics and Aeronautics. American Institute of Aeronautics and Astronautics, (2008).

[7] Iioshi, A., Fujiwara, M., Motjima, O., Ohyabu, N., and Yamazaki, K. Fusion Technology 17, 169-187 (1990).

[8] Toi, K., Ohdachi, S., Sakakibara, S. Y. S., Narihara, K., Tanaka, K., Morita, S., Morisaki, T., Goto, M., Takagi, S., Watanabe, F., Nakajima, N., Watanabe, K. Y., Ida, K., Ikeda, K., Inagaki, S., Kaneko, O., Kawahata, K., Komori, A., Masuzaki, S., Matsuoka, K., Miyazawa, J., Nagaoka, K., Nagayama, Y., Oka, Y., Osakabe, M., Ohyabu, N., Takeiri, Y., Tokuzawa, T., Tsumori, K., Yamada, H., and Yoshinuma, K. Physics of Plasmas 12, 020701-1 - 4 (2005).

[9] Herranz, J., Pastor, I., Castejón, F., de la Luna, E., García-Cortés, I., Barth, C. J., Ascasíbar, E., Sánchez, J., and Tribaldos, V. Phys. Rev. Lett. 85, 47154718 (2000).

[10] Fernandez, A., Sarksyan, K. A., Matveev, N. V., Castejon, F., Cappa, A., Kharchev, N. K., Tereshchenko, M. A., Starshinov, N. N., and Martin, R. Fusion Science and Technology 46, 335-341 (2004).

[11] EFDA. www.efda.org, (2008).

[12] Liu, D. Vertical Compact Torus Injection Into the STOR-M Tokamak. PhD thesis, University of Saskatchewan, (2006).

[13] Parks, P. B. and Perkins, F. W. Nuclear Fusion 46, 770-780 (2006). 
[14] Hwang, D. Q., Horton, R. D., Howard, S., Evans, R. W., and Brockington, S. J. Journal of Fusion Energy 26, 81-84 (2007).

[15] Graf, A., Howard, S., Horton, R., Hwang, D., May, M., Beiersdorfer, P., and Terry, J. Review of Scientific Instruments 77, 10F125 (2006).

[16] Xiao, C., Livingstone, S., Singh, A. K., Zhang, E., and Hirose, A. Plasma Science and Technology 7, 2701-2704 (2005).

[17] Xiao, C., Hirose, A., Zawalski, W., White, D., Raman, R., Decoste, R., Gregory, B., and Martin, F. In IEEE Conference Record. IEEE International Conference on Plasma Science, June (1996).

[18] Raman, R., Gierszewski, P., Thomas, J. C., andG. D. Conway, D. Q. H., Hirose, A., Martin, F., and Decoste, R. Fusion Technology 24, 239-250 (1993).

[19] Raman, R., Martin, F., Quirion, B., St-Onge, M., Lachambre, J. L., Michaud, D., Sawatzky, B., Thomas, J., Hirose, A., Hwang, D., Richard, N., Cote, C., Abel, G., Pinsonneauldt, D., Gauvreau, J. L., Stansfield, B., Decoste, R., Cote, A., Zuzak, W., and Boucher, C. Physical Review Letters 73, 3101-3104 (1994).

[20] Baker, K. L., Hwang, D. Q., Horton, R. D., Evans, R. W., McLean, H. S., and Terry, S. D. In Procedeings of the 18th IAEA Fusion Energy Conference. International Atomic Energy Association, October (2000).

[21] Hwang, D., Horton, R., Evans, R., Howard, S., and Brockington, S. Journal of Fusion Energy 27, 53-56 (2008).

[22] Sugawara, M., Katsumoto, S., Kikuchi, Y., Fukumoto, N., and Nagata, M. In 48th Annual Meeting of the Division of Plasma Physics. American Physical Society, October-November (2006).

[23] Fukumoto, N., Ogawa, H., Nagata, M., Uyama, T., Shibata, T., Kashiwa, Y., Suzuki, S., Kusama, Y., and JFT-2M. Fusion Engineering and Design 81, 2849-2857 (2006).

[24] Parks, P. B. Physical Review Letters 61, 1364-1367 (1988).

[25] Perkins, L. J., Ho, S. K., and Hammer, J. H. Nuclear Fusion 28, 1365-1378 (1988).

[26] Xiao, C., Hirose, A., and Sen, S. Physics of Plasmas 11, 4041-4049 (2004).

[27] Xiao, C., Hirose, A., and Zawalski, W. Nuclear Fusion 38, 249-256 (1998).

[28] Raman, R., Martin, F., Haddad, E., St-Onge, M., Abel, G., Côté, C., Richard, N., Blanchard, N., Mai, H., Quirion, B., LaChambre, J.-L., Hwang, D., Hirose, A., Savoie, S., LeBlanc, B., McLean, H., Xiao, C., Stansfield, B., Côté, A., Michaud, D., and Chartré, M. Nuclear Fusion 73, 967-972 (1997). 
[29] Raman, R. and Gierszewsky, P. Fusion Engineering and Design 4, 977-985 (1998).

[30] Liu, D., Xiao, C., Singh, A. K., and Hirose, A. Nuclear Fusion 46, 104-109 (2006).

[31] Olynyk, G. and Morelli, J. Nucelar Fusion 48, 1-9 (2008).

[32] Hammer, J. H., Hartman, C. W., Eddleman, J. L., and McLean, H. S. Physical Review Letters 61, 2843-2846 (1988).

[33] McLean, H. S., Hwang, D. Q., Horton, R. D., Evans, R. W., and Terry, S. D. Fusion Technology 33, 252-272 (1998).

[34] Bellan, P. M. Spheromaks. Imperial College Press, (2000).

[35] Shimamura, S., Nitou, J., Mayakawa, M., and Oota, F. Journal of Plasma and Fusion Research 6, 492-495 (2004).

[36] Shimamura, S., Nitou, J., and Hayakawa, M. In 30th EPS Conference on Controled Fusion and Plasma Physics, July (2003).

[37] Shimamura, S., Oota, F., Kamata, M., and Asai, T. In 32nd EPS Conference on Plasma Physics, June-July (2005).

[38] Geddes, C. G. R., Kornack, T., and Brown, M. Physics of Plasmas 5, 1027-1034 (1998).

[39] Finn, J. M., Manheimer, W. M., and Ott, E. Physics if Fluids 24, 1336-1341 (1981).

[40] Chen, F. Introduction to Plasma Physics and Controlled Fusion. Springer Science+Business Media, LLC, 2nd edition, (2006).

[41] Marshall, J. Physics of Fluids 3, 134-135 (1960).

[42] Lambda EMI. 402/802 Series High Voltage Power Supplies.

[43] Hutchinson, I. H. Principles of Plasma Diagnostics. Cambridge University Press, 2nd edition, (2002).

[44] Ramboz, J. D. IEEE Transactions on Instrumentation and Measurement 45, 511-515 (1996).

[45] Mott-Smith, H. and Langmuir, I. Physics Review 28, 727-763 (1926).

[46] Matthews, G. F. Plasma Physics and Controlled Fusion 36, 1595-1628 (1994).

[47] Kamitsuma, M., Chen, S.-L., and Chang, J.-S. Journal of Physics D-Applied Physics 10, 1065-1077 (1977). 
[48] Lide, D. R., editor. Handbook of Chemistry and Physics. CRC Press, 82 edition, (2001).

[49] Markusic, T., Berkery, J., and Choueiri, E. IEEE Transactions on Plasma Science 33, 528-529 (2005). 


\section{APPENDix A}

\section{Data Acquisition and Analysis Code}

The MATLAB code that was implemented for all post-processing of the collected data is presented below. Following the MATLAB code, Figure A.1 provides the NI Labview program used to acquire and save data from the digitizer.

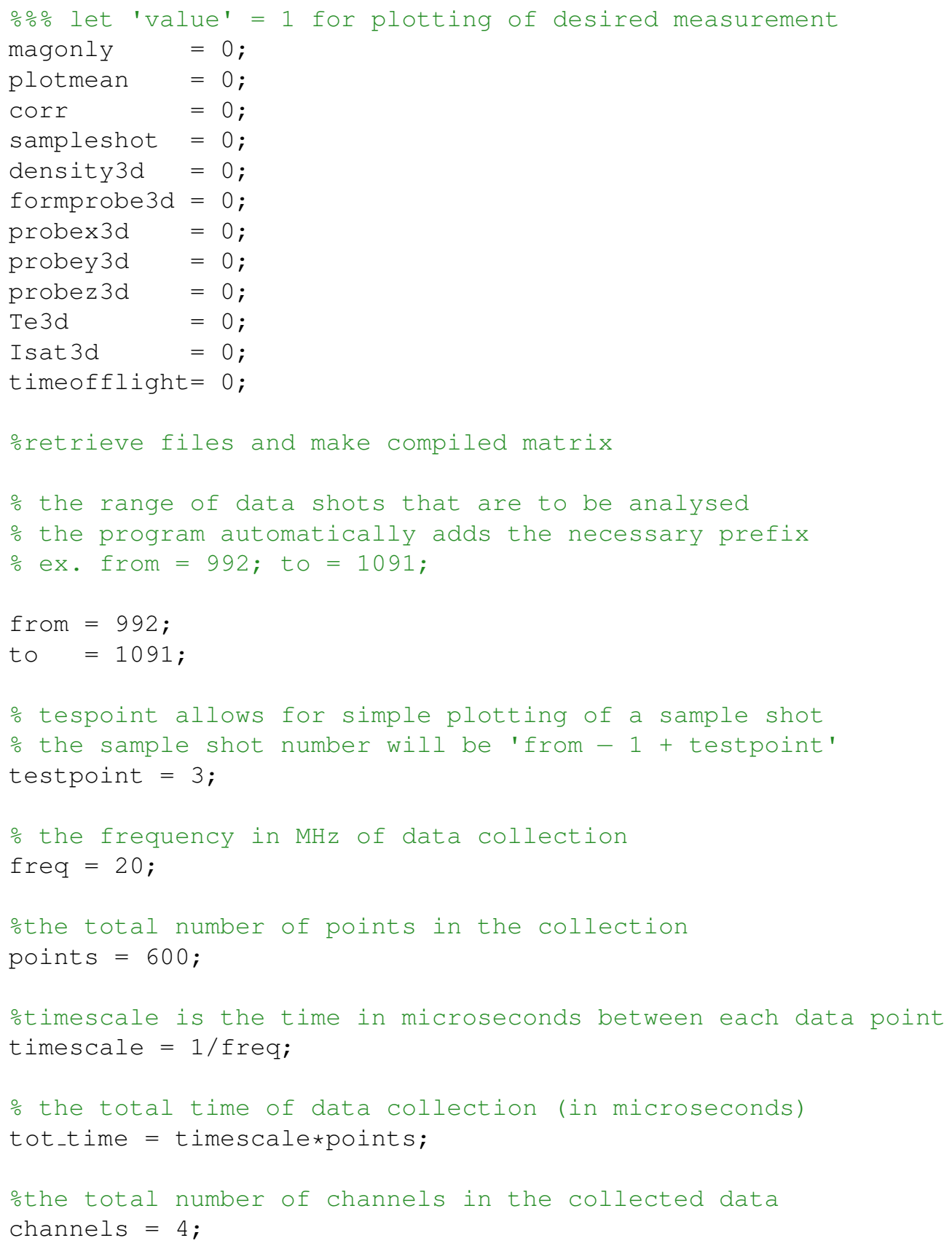




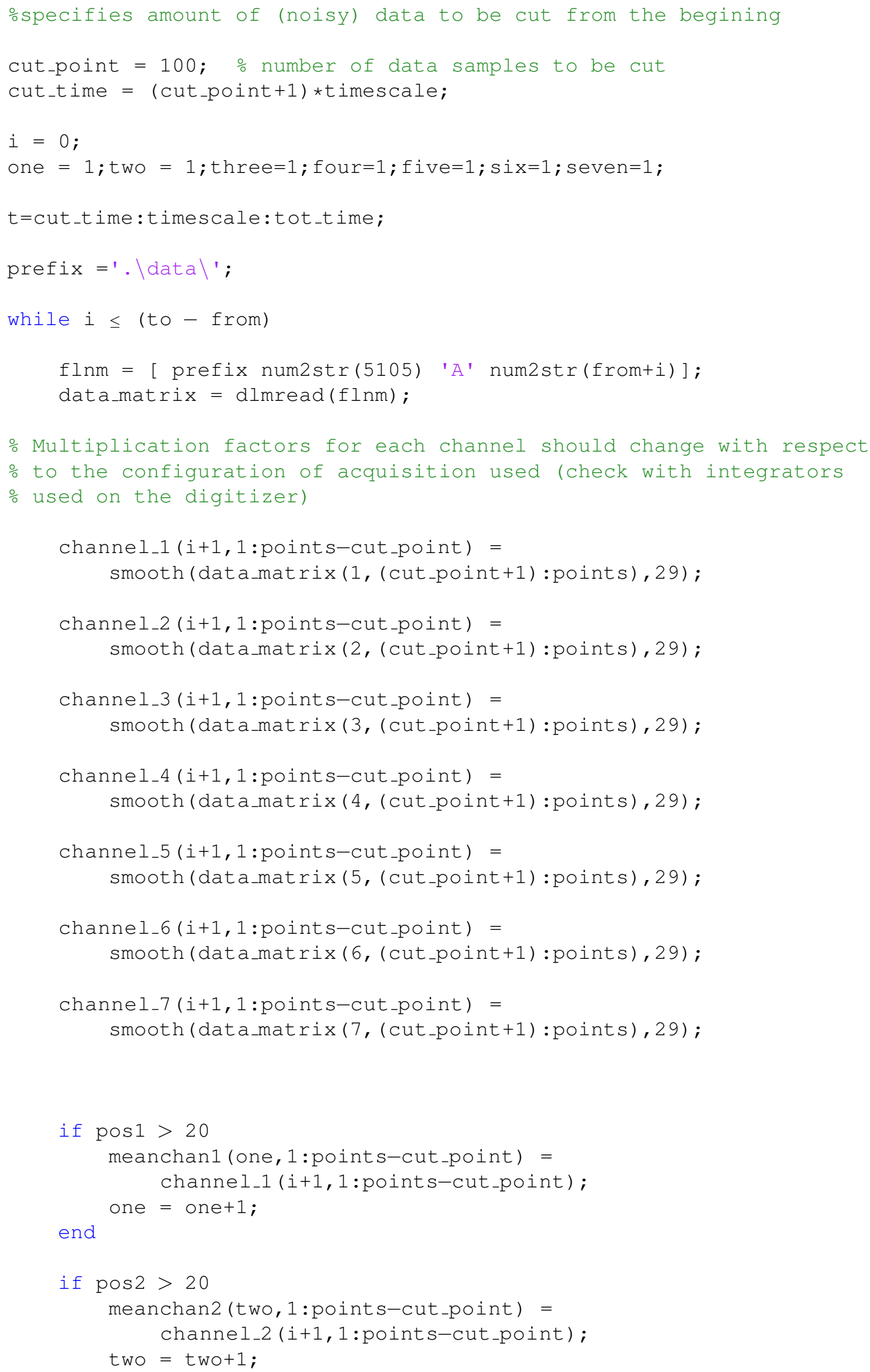




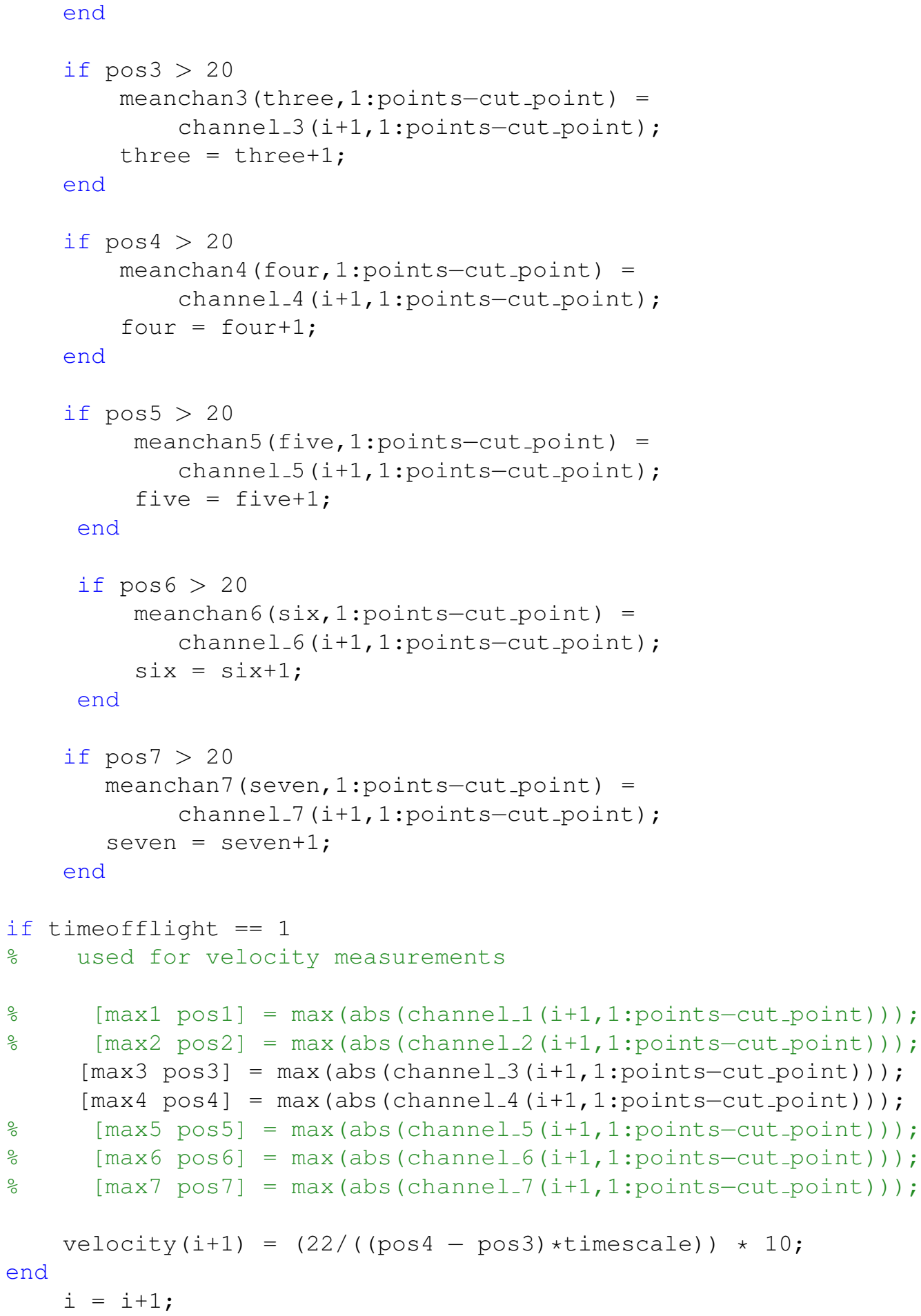

end

Num_shots $=1: 1: i$;

if timeofflight $==1$

flight $=1$ 


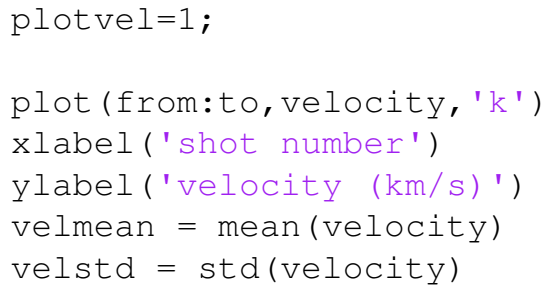




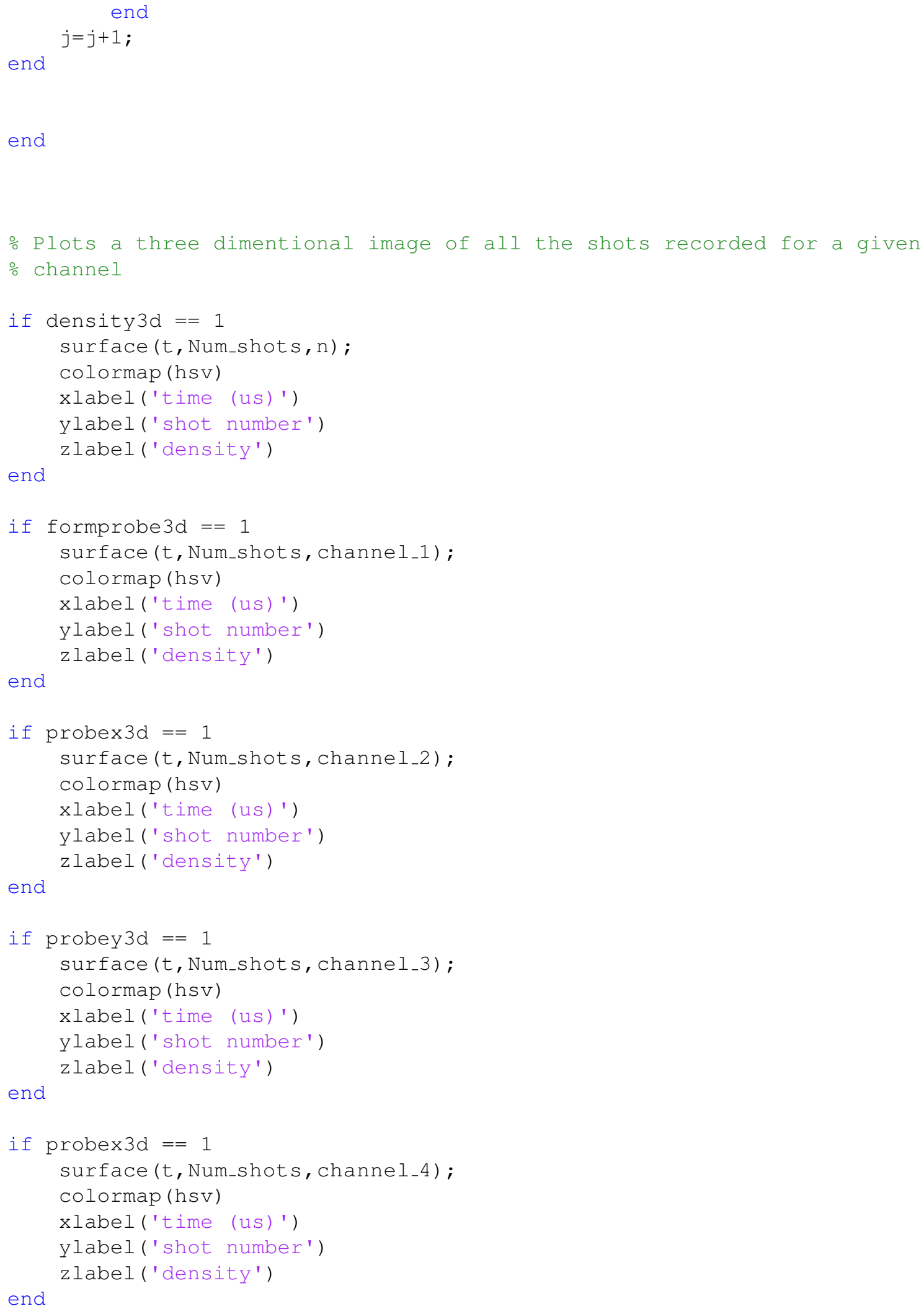




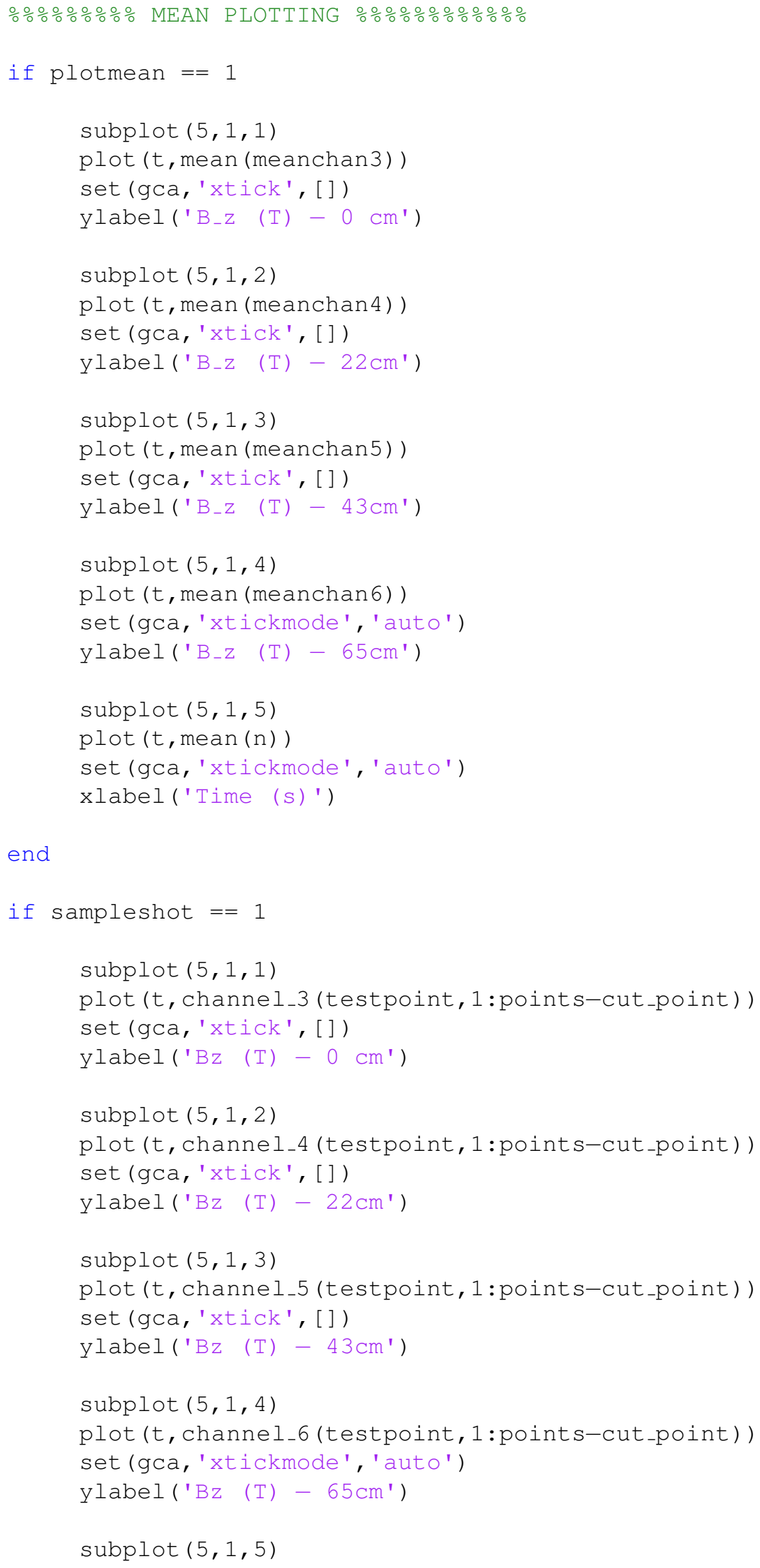


plot (t,n (testpoint, 1:points-cut_point))

set (gca, 'xtickmode', 'auto')

xlabel ('Time (s)')

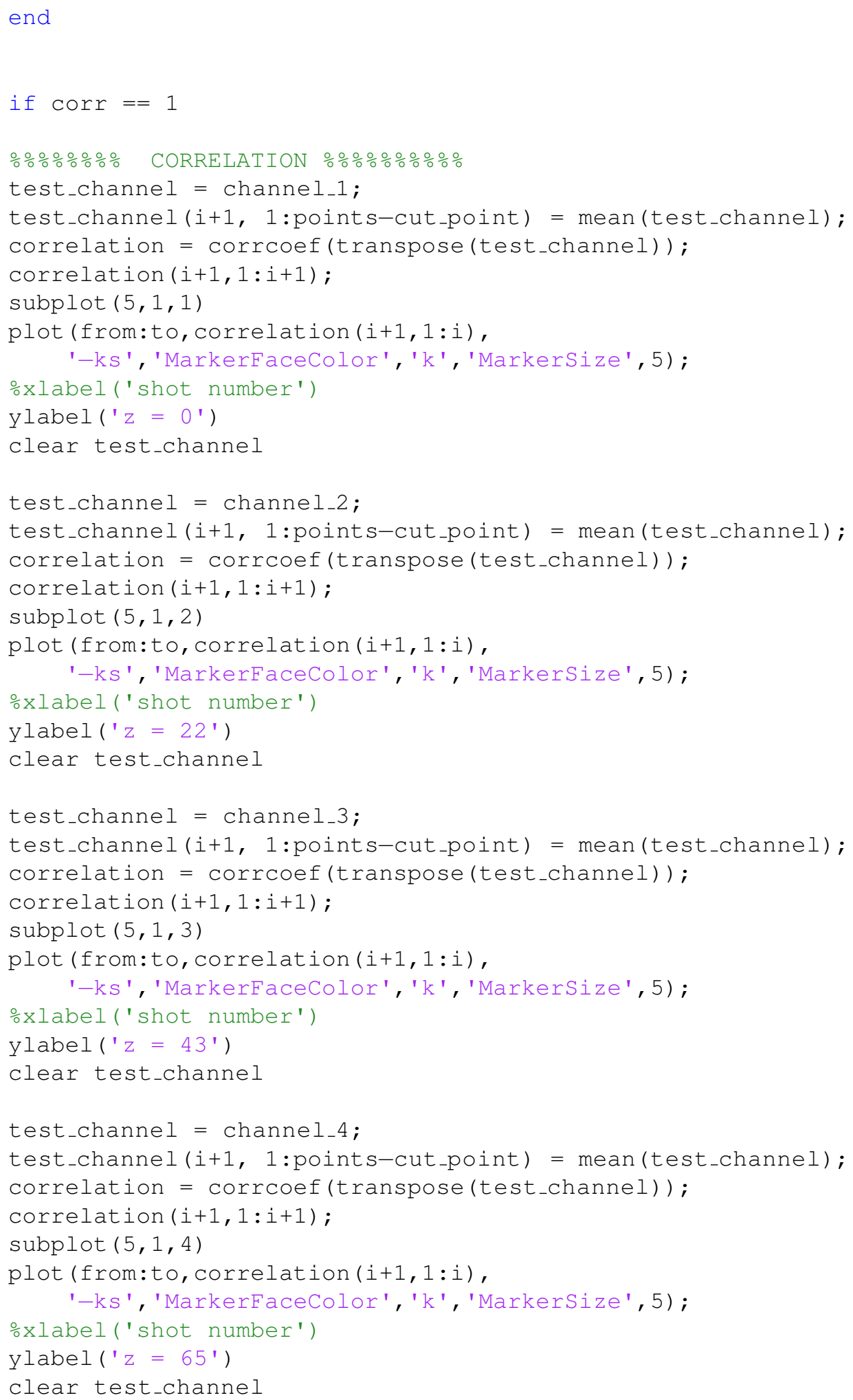




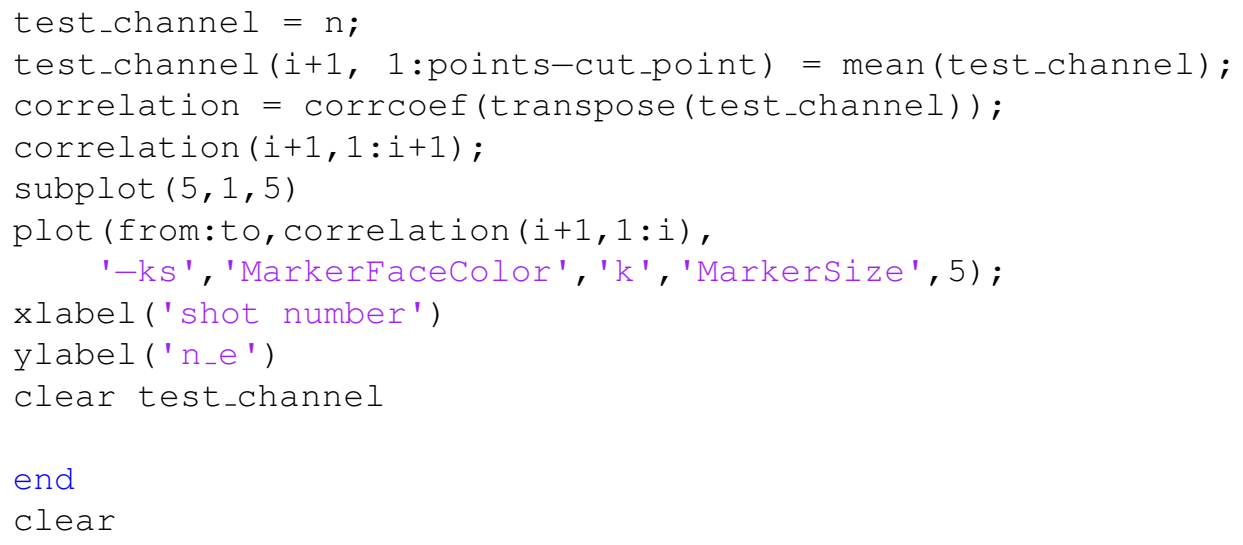




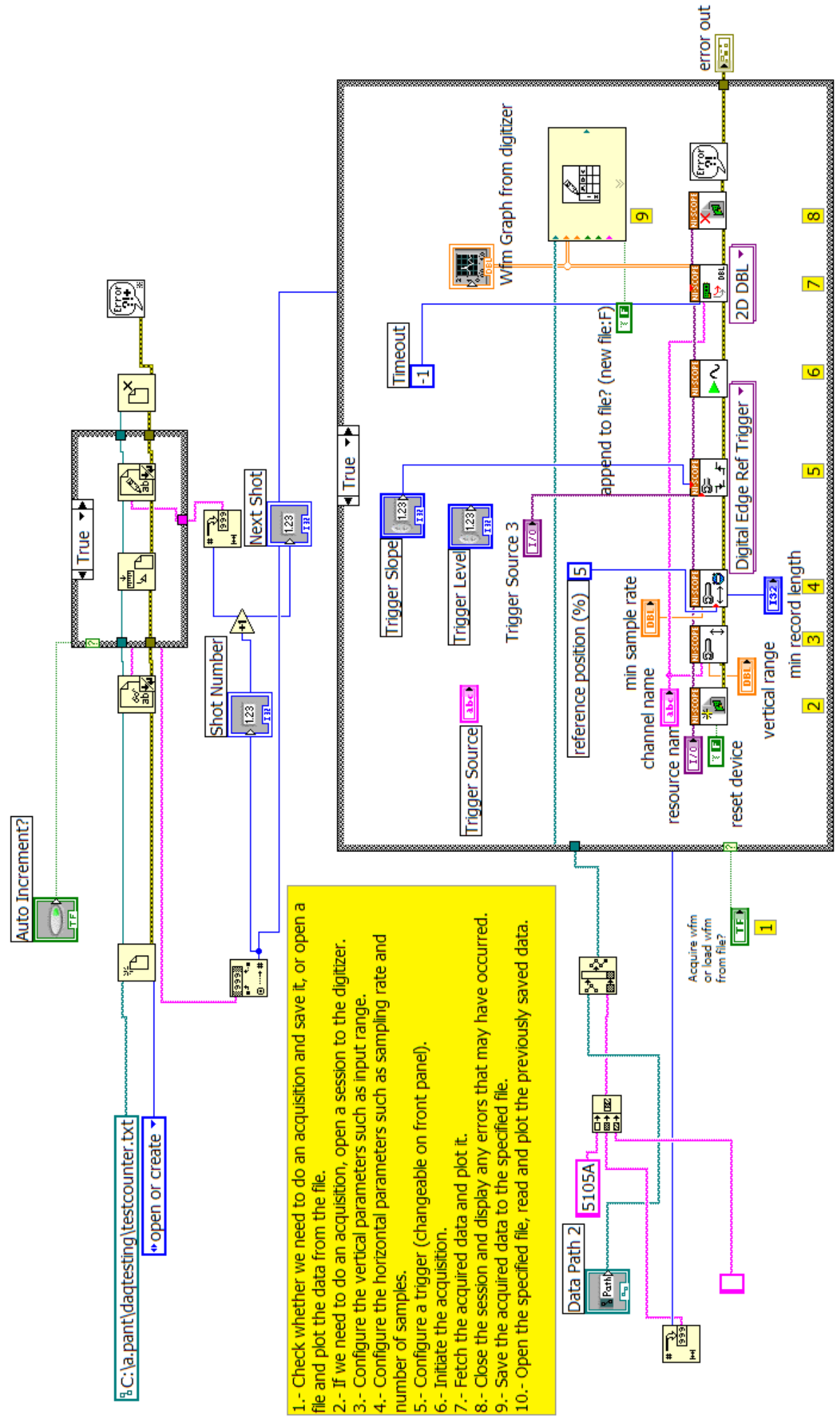

Figure A.1: Labview acquisition software. 